\title{
Mesoscopic electrodynamics at metal surfaces
}

From quantum-corrected hydrodynamics to microscopic surface-response formalism

\section{Mortensen, N. Asger}

\section{Published in:}

Nanophotonics

Link to article, DOI:

10.1515/nanoph-2021-0156

Publication date:

2021

\section{Document Version}

Publisher's PDF, also known as Version of record

Link back to DTU Orbit

\section{Citation (APA):}

Mortensen, N. A. (2021). Mesoscopic electrodynamics at metal surfaces: From quantum-corrected hydrodynamics to microscopic surface-response formalism. Nanophotonics, 10(10), 2563-2616. https://doi.org/10.1515/nanoph-2021-0156

\section{General rights}

Copyright and moral rights for the publications made accessible in the public portal are retained by the authors and/or other copyright owners and it is a condition of accessing publications that users recognise and abide by the legal requirements associated with these rights.

- Users may download and print one copy of any publication from the public portal for the purpose of private study or research.

- You may not further distribute the material or use it for any profit-making activity or commercial gain

- You may freely distribute the URL identifying the publication in the public portal 


\title{
Review
}

\section{N. Asger Mortensen*}

\section{Mesoscopic electrodynamics at metal surfaces}

\author{
- From quantum-corrected hydrodynamics to microscopic surface-response formalism
}

https://doi.org/10.1515/nanoph-2021-0156

Received April 12, 2021; accepted June 4, 2021;

published online June 25, 2021

\begin{abstract}
Plasmonic phenomena in metals are commonly explored within the framework of classical electrodynamics and semiclassical models for the interactions of light with free-electron matter. The more detailed understanding of mesoscopic electrodynamics at metal surfaces is, however, becoming increasingly important for both fundamental developments in quantum plasmonics and potential applications in emerging light-based quantum technologies. The review offers a colloquial introduction to recent mesoscopic formalism, ranging from quantumcorrected hydrodynamics to microscopic surface-response formalism, offering also perspectives on possible future avenues.
\end{abstract}

Keywords: Landau damping; nonlocal electrodynamics; quantum-corrected mesoscopic boundary conditions; quantum plasmonics; semi-classical hydrodynamics; surface-response formalism.

\section{Introduction}

The modern information society is largely enabled by a revolution in information and communication technologies, being strongly rooted in classical electrodynamics and further catalyzed by developments in quantum physics, condensed-matter physics, optical sciences, and materials science. While electrodynamics is largely a classical discipline, the developments of quantum mechanics immediately stimulated a curiosity for how to connect and

\footnotetext{
*Corresponding author: N. Asger Mortensen, Center for Nano Optics, University of Southern Denmark, Campusvej 55, DK-5230 Odense M, Denmark; Danish Institute for Advanced Study, University of Southern Denmark, Campusvej 55, DK-5230 Odense M, Denmark; and Center for Nanostructured Graphene, Technical University of Denmark, DK-2800 Kongens Lyngby, Denmark, E-mail:asger@mailaps.org._https://orcid.org/0000-0001-79366264
}

¿ Open Access. @2021 N. Asger Mortensen, published by De Gruyter. (ब) BY License. understand physical phenomena and observables across the border between the more familiar properties of the world of classical physics and the new intriguing world governed by quantum physics. This constitutes a longlasting curiosity [1], and this review joins this curiosity with a focus on explorations of light-matter interactions at exactly this interface between the classical electrodynamics of electromagnetic fields and the quantum physics of condensed-matter systems. In particular, a mesoscopic electrodynamic formalism for plasmonics systems is being reviewed, including both semiclassical hydrodynamics and microscopic surface-response formalism, all aiming to correct the classical electrodynamics for quantum effects occurring at metal surfaces. Beyond the mere curiosity in understanding fundamental electrodynamic phenomena of matter - ranging from two-dimensional (2D) materials to strongly-correlated materials - the mesoscopic electrodynamics formalism also has implications for emerging quantum technologies in the second quantum revolution $[2,3]$, including the use of quantum plasmonics [4-7] to enhance the generation of nonclassical light for quantuminformation processing [8-10].

The review is written in a colloquial style, aiming to offer a broad introduction to the mesoscopic electrodynamics at metal surfaces, hopefully serving as a useful starting point for scientists at all levels with a more general interest in electrodynamics, while also providing an overview to experts in plasmonics.

\subsection{Foundation of classical electrodynamics}

During 2020, we were celebrating the 200-years anniversary of Ørsted's discovery in electromagnetism that he disclosed on July 21, 1820 [11]. His observation of the mutual connection of electrical currents and magnetic fields contributed to and further inspired curiosity-driven discoveries by several European contemporaries in physics and mathematics, including Ampère, Coulomb, Faraday, Ohm, and Gauss [12]. Eventually, this inspired Maxwell to formulate his now-famous set of four partial differential equations (PDE) [13] that form the basis for our 
fundamental understanding of classical electromagnetism [14]:

$$
\begin{gathered}
\boldsymbol{\nabla} \cdot \boldsymbol{D}(\boldsymbol{r}, t)=\rho(\boldsymbol{r}, t), \\
\boldsymbol{\nabla} \cdot \boldsymbol{B}(\boldsymbol{r}, t)=0, \\
\boldsymbol{\nabla} \times \boldsymbol{E}(\boldsymbol{r}, t)=-\partial_{t} \boldsymbol{B}(\boldsymbol{r}, t), \\
\boldsymbol{\nabla} \times \boldsymbol{H}(\boldsymbol{r}, t)=\boldsymbol{J}(\boldsymbol{r}, t)+\partial_{t} \boldsymbol{D}(\boldsymbol{r}, t) .
\end{gathered}
$$

In order of appearance, $\boldsymbol{D}$ is the displacement field, $\rho$ is the electric charge density, $\boldsymbol{B}$ is the magnetic field, $\boldsymbol{E}$ is the electric field, $\boldsymbol{H}$ is the magnetizing field, and $\boldsymbol{J}$ is the electric current density - all scalar and vectorial fields having spatial and temporal variations as indicated by the spatial coordinate vector $\boldsymbol{r}$ and the temporal coordinate $t$, respectively. These equations of Maxwell have remained a cornerstone in physics and engineering ever since and his formalism has formed the foundation for numerous scientific discoveries and technological innovations [15]. Slightly more than 150 years later, one can hardly overestimate the importance of Maxwell's equations to modern society. In fact, electromagnetism remains a fantastic playground that still catalysis surprising developments [16] that for sure must have progressed far beyond any imagination of Maxwell himself.

A later important contribution to electrodynamics came from Lorentz, describing the electromagnetic force (now known as the Lorentz force) exerted on moving electrically charged particles by electrical and magnetic forces. In some way, Lorentz also prepared the ground for later important developments (by Einstein, Bohr, and many contemporaries) in both relativity and quantum physics. The latter paradigm has been tremendously important for our understanding of matter and light-matter interactions, including atomic physics and extensions to perhaps even more complex condensed-matter systems comprised by manifolds of such atoms. Alongside this development, there have been numerous advances in the classical, semiclassical, and quantum descriptions of matter and the constitutive laws invoking the mutual interaction with light, while the electromagnetic fields themselves remain being governed by Maxwell's equations. Even when the quantum nature of light, i.e. photons, is considered, Maxwell's equations still remain as a basis for the introduction of quantized light fields. As such, the overall developments within electrodynamics have not seriously rocked the foundation itself, but rather the explorations and applications of electrodynamics have been further fueled by developments in materials science and micro/nanotechnology (including materials synthesis, materials processing, and device fabrication) combined with new predictive power offered by computational physics and advanced mathematics.

\subsection{Electrodynamics in modern optical sciences}

In the optical sciences (the physical science of light and light-matter interactions), being mainly engaged with the optical frequency part of the even broader electromagnetic spectrum, the developments surrounding electromagnetic waves have in recent decades catalyzed the growth of a number of new concepts in photonics, all rooted in the classical electrodynamics of Maxwell's equations. The examples are including fiber optics [17], photonic crystals $[18,19]$, plasmonics (which will be addressed in more details), transformation optics and metamaterials [20-22], metasurfaces [23, 24], zero-index materials [25-27], 2D material photonics [28-34], and most recently nonHermitian photonics [35-37] and topological photonics [38-40]. Perhaps there is no need to emphasize, but the breath of photonics is naturally even broader (including of course also light generation, manipulation, and detection) and today it is recognized as a key enabling technology of critical importance to society.

For the further harvesting of the full potential of photonics, the field has so far mainly exploited the more (semi)classically based properties of optical materials and light fields $[8,10]$. Naturally, one needs the quantum descriptions of electrons in solids to appreciate the optical properties of semiconductors and metals. However, when eventually interfacing Maxwell's equations, the materials' properties usually enter through quite simple constitutive laws:

$$
\begin{aligned}
& \boldsymbol{D}(\boldsymbol{r})=\varepsilon(\boldsymbol{r}, \omega) \boldsymbol{E}(\boldsymbol{r}), \\
& \boldsymbol{B}(\boldsymbol{r})=\mu(\boldsymbol{r}, \omega) \boldsymbol{H}(\boldsymbol{r}), \\
& \boldsymbol{J}(\boldsymbol{r})=\sigma(\boldsymbol{r}, \omega) \boldsymbol{E}(\boldsymbol{r}) .
\end{aligned}
$$

Here, we are for convenience emphasizing the Fourier frequency domain associated with temporal harmonic fields with an $\mathrm{e}^{-\mathrm{i} \omega t}$ time dependence (where $\omega$ is the angular frequency), while for ease of notation we have suppressed the frequency dependence of the fields. In other words, the electromagnetic properties of materials are being characterized by their electric permittivities $\varepsilon$, magnetic susceptibilities $\mu$, and electrical conductivities $\sigma$. At this stage, these are largely phenomenologically based response functions. 
For the magnetic response (Eq. (2b)) it is common to use $\mu(\boldsymbol{r}, \omega) \simeq \mu_{0}$, where $\mu_{0}$ is the vacuum permeability. This approximation (to be utilized throughout the remaining of this review) works extremely well at optical frequencies, where magnetic moments are reacting too slowly to the rapidly varying optical fields to cause any appreciable magnetic response. Maxwell's equations (Eqs. (1)) can now be rewritten as a generalized eigenvalue problem:

$$
\boldsymbol{\nabla} \times \boldsymbol{\nabla} \times \boldsymbol{E}(\boldsymbol{r})=\left(\frac{\omega}{c}\right)^{2}\left[\frac{\varepsilon(\boldsymbol{r}, \omega)}{\varepsilon_{0}}-\frac{\sigma(\boldsymbol{r}, \omega)}{i \varepsilon_{0} \omega}\right] \boldsymbol{E}(\boldsymbol{r}) .
$$

Here, $c=\left(\varepsilon_{0} \mu_{0}\right)^{-1 / 2}$ is the speed of light in vacuum and $\varepsilon_{0}$ is the vacuum permittivity. It is worth noting how the response of the free electrons can be represented by also a dielectric function by mathematically absorbing $\sigma$ into $\varepsilon$. At this stage, it is, however, instructive to maintain both terms, relating interband transitions (localized/bound electrons) to the $\varepsilon$ term, while accounting for intraband transitions (free electrons) through the $\sigma$ term.

For dielectrics - high-quality optical materials with low absorption - it is common to use semiclassical Drude-Lorentz models for the dielectric function $\varepsilon(\boldsymbol{r}, \omega)$ (Eq. (2a)) or alternatively experimentally tabulated materials properties, potentially fitted with the Drude-Lorentz model or Sellmeier expressions. In the presence of resonances, causality implies that the dielectric function is complex-valued $\left(\varepsilon=\varepsilon^{\prime}+i \varepsilon^{\prime \prime}\right)$, with the real and imaginary parts being related through Kramers-Kronig relations [41]. However, in many frequency regimes and away from absorption resonances, it is common to simply neglect any small frequency dispersion, thus approximating the dielectric function by a real-valued constant, $n^{2}=\varepsilon \approx \varepsilon^{\prime}$, where $n$ is the refractive index.

For the electrodynamics of free electrons in metals, the use of a linear conductivity $\sigma(\boldsymbol{r}, \omega)$ and Ohm's law (Eq. (2c)) is being introduced already in the physics classes of primary school. As such, we may tend to simply take it for granted. On the other hand, it appears almost pedestrian when compared to developments in other research areas of nanoscience, e.g. mesoscopic electron transport and molecular electronics, where the transport of charge can be ballistic [42]. Ohm's law assumes a local linear-response relation between electrical currents $\boldsymbol{J}(\boldsymbol{r}, \omega)$ induced in a given point $\boldsymbol{r}$ in response to driving electrical fields $\boldsymbol{E}(\boldsymbol{r}, \omega)$ in the very same point $\boldsymbol{r}$. In other words, there is no longrange response of the electron gas due to the perturbation. A priori, there is nothing wrong with Ohm's law, and in most cases, this intuitive form is an appropriate and accurate description of the interaction of electromagnetic fields with free charge carriers. In fact, the accuracy very much depends on the underlying description of the material's conductivity that enters Ohm's law, and in most cases, we are anyway utilizing experimentally tabulated values of materials' properties in the frequency regimes of interest.

This review has a focus on physics that manifest in experimentally more recently accessible regimes, where the above form of Ohm's law falls short in accurately accounting for the underlying quantum and nonlocal dynamics of electrons. On such situations, the constitutive relations generalize to integral forms [14]:

$$
\begin{gathered}
\boldsymbol{D}(\boldsymbol{r})=\int \mathrm{d} \boldsymbol{r}^{\prime} \varepsilon\left(\boldsymbol{r}, \boldsymbol{r}^{\prime}, \omega\right) \boldsymbol{E}\left(\boldsymbol{r}^{\prime}\right), \\
\boldsymbol{J}(\boldsymbol{r})=\int \mathrm{d} \boldsymbol{r}^{\prime} \sigma\left(\boldsymbol{r}, \boldsymbol{r}^{\prime}, \omega\right) \boldsymbol{E}\left(\boldsymbol{r}^{\prime}\right) .
\end{gathered}
$$

Here, the finite range of $\sigma\left(\boldsymbol{r}, \boldsymbol{r}^{\prime}, \omega\right)$ quantifies the degree of nonlocality in the free electrons' response to driving fields [43]. Mathematically, this linear-response integral form originates from a Volterra series, which generalizes the more common Taylor series by its ability to capture temporospatial "memory" effects, i.e. temporal and spatial dispersion [44]. The PDE in Eq. (3) now generalizes to the integro-differential equation (IDE):

$$
\begin{aligned}
\boldsymbol{\nabla} \times \boldsymbol{\nabla} & \times \boldsymbol{E}(\boldsymbol{r}) \\
& =\left(\frac{\omega}{c}\right)^{2} \int \mathrm{d} \boldsymbol{r}^{\prime}\left[\frac{\varepsilon\left(\boldsymbol{r}, \boldsymbol{r}^{\prime}, \omega\right)}{\varepsilon_{0}}-\frac{\sigma\left(\boldsymbol{r}, \boldsymbol{r}^{\prime}, \omega\right)}{i \varepsilon_{0} \omega}\right] \boldsymbol{E}\left(\boldsymbol{r}^{\prime}\right) .
\end{aligned}
$$

We shall return briefly to the mathematical and physical foundation for these generalized linear-response constitutive laws. At this point, it suffices to note that the strength of linear-response theory is that the phenomenological coefficient $\sigma$, that emerges in the linear Taylor expansion $\boldsymbol{J}(\boldsymbol{E}) \simeq 0+\sigma \boldsymbol{E}$, can usually be determined from experiments. Obviously, there is a widespread tabulation of metals' conductivities, implicitly assuming the localresponse approximation (LRA). As an example, the lowfrequency conductivity of metals is well documented for centuries and the high-frequency optical response is also known, e.g. from the tables of optical constants of the noble metals by Johnson and Christy [45]. However, there is currently no tabulated experimental data available for the corresponding two-point response function.

The aim of recent efforts has been to establish a theoretical understanding of $\sigma\left(\boldsymbol{r}, \boldsymbol{r}^{\prime}, \omega\right)$ [or alternatively $\left.\varepsilon\left(\boldsymbol{r}, \boldsymbol{r}^{\prime}, \omega\right)\right]$ and its implications for optical experiments that invoke metal nanostructures hosting plasmon excitations, i.e. collective oscillations in the free-electron gas. This is a rapidly developing subfield within the broader area of nano-optics [46, 47] and plasmonics [48] (the science and technology associated with light fields interacting with 
plasmons and nanoscale light-matter interactions), being commonly referred to as nano plasmonics, and recently even quantum plasmonics in the anticipation of optical manifestation of also quantum phenomena [5].

The nonlocal aspects of plasmons were already implicit in the hydrodynamic description of electrons by Bloch [49], and since then it is well-established within condensed-matter physics that $\sigma$ is a two-point function. However, for historical reasons, implications have mainly been explored for extended and translational invariant systems where semiclassical and quantum descriptions are available within the Fourier-space representation $[50,51]$, i.e. with a focus on $\sigma(\boldsymbol{q}, \omega)$. The Lindhard dielectric function $\varepsilon(\boldsymbol{q}, \omega)$ is a prime example of a quantum mechanical description of the linear-response of electrons in metals [52, 53], but given in a Fourier-space representation that challenges its immediate real-space application to finite nanostructures without translation invariance.

\subsection{Plasmonics}

As already alluded to in Section 1.2, the term plasmonics covers the optical science and photonics technology associated with the interaction of light with plasmons. Obviously, the name brings associations with the term electronics, referring to technologies and devices that harvest from the properties of electrons. While electronics largely operate through the single-particle manipulation of excitations (of fermionic nature) in the free-electron gas, the plasmon is a collective excitation (of bosonic nature) involving all the free electrons. Plasmons propagate as charge-density waves, much resembling the propagation of sound in a compressible gas of molecules.

Following the discovery of Langmuir waves in ionized gases in the 1920s [54], the existence of plasmons in solids was predicted in the 1950s by Pines and Bohm [55-59]. As suggested in the above analogy with sound waves, plasmons are charge-density waves that propagate in the bulk of metals are of a longitudinal kind. However, near surfaces of metals, the spatial termination of the electron gas allows for spatially localized plasmon excitations - so-called surface plasmons (SPs), which are transverse electromagnetic waves being strongly confined normal to the surface, while free to propagate along the surface [60]. Their hybridization with light fields is commonly referred to as surface-plasmon polaritons (SPPs). The SP was in 1957 theoretically predicted by Ritchie [61] and shortly after experimentally observed by Powell and Swan [62].

It is the concept of SPs that forms the backbone of modern plasmonics, now branching out in many interesting research directions and application areas [63-65].
There are by now many basic introductions to plasmonics, including the textbooks by Maier [66] (reflecting the early focus on more common metals like the noble metals) and Gonçalves and Peres [67] (extending the introduction to also include the more recent 2D materials). To get an impression of the current breath of plasmonics, the reader is referred to the special issue [68] published in connection with the 9th International Conference on Surface Plasmon Photonics (SPP9) held recently in Copenhagen (www.SPP9.dk). Above, we have emphasized the plasmon response associated with intraband transitions of free carriers. Beyond metals, plasmons can of course also manifest in less dense electron systems, and as such, plasmonics is also explored in doped semiconductors [69-71] and 2D materials [32, 34, 72]. In passing, we note that there is now also growing attention to plasmon-like behavior associated with negative dielectric function values. This can be due to electronic interband transitions, including the possible interband response in graphene $[30,67,73]$ and in silicon [74], while the negative response may also be caused by phonon polaritons, such as in polar dielectrics [75].

Plasmonics is a research area and application field that has developed tremendously with a foundation in classical electrodynamics. Figure 1 is illustrating some of the most common approximations involved in the classical electrodynamics treatment $[48,66]$, some of which are discussed briefly in the following while being revisited when reviewing the mesoscopic electrodynamics formalism that seeks to relax several of those inherent approximations. While still relying on Maxwell's equations and common linear-response theory, the further approximations may be relaxed by resorting to material-response models of increasing complexity, ranging from the semiclassical nonlocal hydrodynamic formalism [76] and Boltzmann formalism [50] over quantum many-body formalism [77], such as the random-phase approximation (RPA) and Lindhard theory [52] or Kubo formalism [78], or alternatively $a b$ initio computational approaches widespread in condensed-matter physics, such as time-dependent density-functional theory (TDDFT) [79]. These methods not only enable detailed descriptions of bulk response functions but also mesoscopic surface properties that can be rigorously accounted for in the electrodynamics through surface-response formalism [80].

\subsubsection{Piecewise-constant approximation}

When considering plasmons in metal structures of a finite extension, one needs to describe the dielectric function 


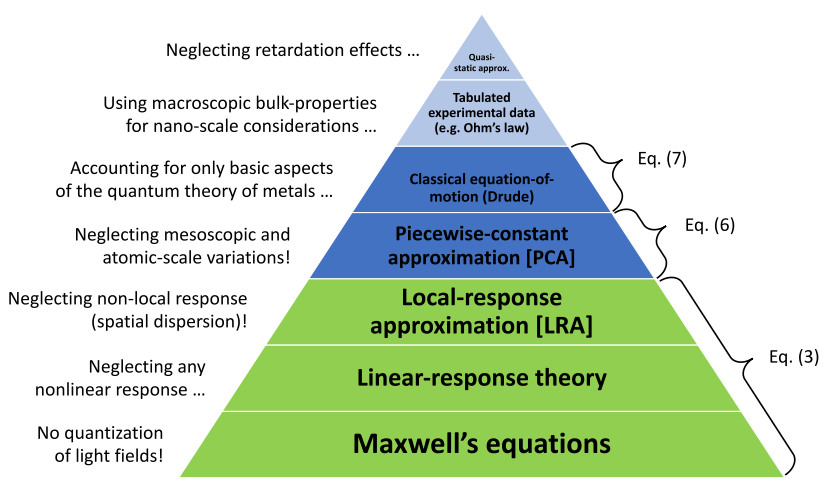

Figure 1: Schematic summary of common approximations inherent to the classical electrodynamics treatment of plasmonic phenomena at surfaces of metallic nanostructures, highlighting also examples of intriguing physical effects being neglected - some of these constitute entire research areas in themselves, such as quantum optics and nonlinear optics, while others represent aspects of the quantum regime addressed by mesoscopic electrodynamics formalism. Mathematical implications of the classical electrodynamics are discussed in, e.g. Eqs. (3), (6), and (7).

in both the interior and the exterior of the metal volumes. Inspired by long-established traditions in the electrodynamics of composite dielectric problems [81], it is in plasmonics likewise common to invoke yet another approximation - piecewise-constant approximation (PCA) with an abrupt surface termination of the metal, where we neglect any atomic-scale inhomogeneities in the vicinity of the surface $[48,66]$

$$
\varepsilon(\boldsymbol{r}, \omega) \approx\left\{\begin{array}{cc}
\varepsilon(\omega), & \boldsymbol{r} \in \text { metal, } \\
\varepsilon_{0}, & \boldsymbol{r} \notin \text { metal, }
\end{array}\right.
$$

while similar considerations apply to $\sigma(\boldsymbol{r}, \omega)$. As a result, the dielectric landscape is now formed by interfacing piecewise-constant (bulk) local-response functions, while Eq. (3) is then solved by invoking the classical pillbox electrodynamic boundary conditions at these interfaces [14]. It should be emphasized that the PCA has been tremendously successful in advancing the field of plasmonics, where it has proven absolutely sufficient to interpret the majority of experimentally observed phenomena. On the other hand, the same approximations are being challenged by more recent developments in nanoscale plasmonics and light-matter interactions [10, 82-84].

\subsubsection{The Drude model underlying plasmonics}

To appreciate the mere existence of plasmons, the vast majority of works in plasmonics start from the Drude description of the dynamics of free electrons exposed to an electrical field [85]. The starting point is entirely classical, utilizing Newton's second law to establish an equation of motion for a single free electron, while subject to the electrical field $\boldsymbol{E}$ in the Lorentz force:

$$
m \partial_{t} \boldsymbol{v}=-\gamma m \boldsymbol{v}-e \boldsymbol{E} .
$$

Here, $\boldsymbol{v}$ is the velocity field of the electron which has the electrical charge $e$. In the general case of free electrons, $m$ would be the common mass of the electron, while when later dealing with electrons in a solid, it becomes the effective mass [86]. The right-hand side contains also a relaxation term $\gamma \boldsymbol{v}$ associated with the scattering time $\tau=\gamma^{-1}$ of the electrons. Only later, the quantum description of the electron gas makes its entry, when semiclassically accounting for the sea of free electrons contributing to the collective plasmon oscillation.

For time-harmonic fields, Eq. (7) is solved straightforwardly, giving the velocity field $\boldsymbol{v} \propto \boldsymbol{E}$ of the electron. Only now, the quantum theory of the electron gas enters, when summing up the contributions from many such electrons to form a current density $\boldsymbol{J}=-n e \boldsymbol{v}$, where $n$ is the density of free conduction electrons in the conduction band of the metal [86]. In passing, we note that rather than a continuum formulation, one may also follow a computationally more challenging particle-simulation approach, where one aims to keep track of the many individual conduction electrons $[87,88]$. Following the continuum formulation, the end result is nothing but Ohm's law, but now with a semiclassical expression for the frequency-dispersive conductivity [86]

$$
\sigma(\omega)=i \varepsilon_{0} \frac{\omega_{p}^{2}}{\omega+i \gamma},
$$

or alternatively the associated equivalent dielectric function [86]

$$
\varepsilon(\omega)=1-\frac{\omega_{p}^{2}}{\omega(\omega+i \gamma)} .
$$

Here, $\omega_{p}=\sqrt{n e^{2} / m \varepsilon_{0}}$ is the characteristic plasma frequency associated with collective oscillation of the electron gas. It is also the frequency where $\varepsilon(\omega)$ changes sign, being negative below the plasma frequency while attaining a positive value above. Note that for later ease of notation, Eq. (8) is now omitting $\varepsilon_{0}$, so that $\varepsilon(\omega)$ is being the relative dielectric function, with its corresponding value in a vacuum being given by $\varepsilon=1$.

To better appreciate that $\omega_{p}$ constitutes a characteristic frequency of the plasma, solving Eq. (3) for longitudinal fields $(\boldsymbol{\nabla} \times \boldsymbol{E}=0$ ) gives the well-known condition $\varepsilon(\omega)=0$ for the existence of longitudinal plasmon oscillations. The solution is obviously an oscillation at the frequency 
$\omega_{L}(\boldsymbol{q})=\omega_{p}$. This excitation is known as the bulk plasmon. The absence of any wave vector dispersion is an immediate effect of the LRA. For transverse fields $(\boldsymbol{\nabla} \cdot \boldsymbol{E}=0)$, the solution is $\omega_{T}(\boldsymbol{q})=\sqrt{\omega_{p}^{2}+c^{2} q^{2}}$, making the plasma transparent to propagating waves only above the plasma frequency, while propagation is prohibited below the plasma frequency. It should be emphasized that here we have entirely focused on the response of the free conduction electrons, while deliberately leaving out contributions from bound electrons to the dielectric function, since this can be accounted for straightforwardly by an additional background dielectric function. For transparency and the ease of presentation, this choice is maintained throughout the review, except when turning to computational examples involving e.g. noble metals.

\subsubsection{Excitation of plasmons}

Since plasmons involve the displacement of charged particles, they may quite naturally couple to the electromagnetic fields of Maxwell's equations. As an example, the electrical field, associated with light impinging on a metallic nanostructure, will exert a force on the free electrons - under the right circumstances, this can excite a plasmon oscillation, and in turn, this will be reflected also in the spectral composition of the scattered light. As such, the existence of plasmons can be physically manifested and optically observed in the appearing color of the nanoparticles [63, 89, 90]; an effect known from ancient glass [91, 92] and now exploited with modern nanotechnology for artificial colors [93, 94].

As an alternative to the intuitive excitation by light waves, e.g. beams of photons as in a classical light microscope, plasmons can likewise be excited by beams of charged particles, e.g. with electron beams in an electron microscope. The coupling of the incident electrons with the plasmons can naturally be appreciated from the repulsive force in Coulomb's law, while one can also associate electromagnetic fields with the impinging flux of electrons. Historically, excitation by swift electrons was the first means to experimentally observe plasmons [62], while the majority of later studies and applications invoke light excitation schemes. Naturally, the diffraction limit of Abbe [95] enforces some hard resolution limitations to the observation of plasmon phenomena in optical spectroscopy, thus limiting the ability to spatially resolve plasmons at sub-wavelength length scales in true nanostructures. However, amazing developments in electron-based spectroscopies $[96,97]$ have over the recent decades opened complementary ways for explorations of plasmons all the way to the few nanometer length scales. As an example, plasmons have been observed in $L \sim 500 \mathrm{~nm}$ silver nanowires [98] with electron-energy loss spectroscopy (EELS) in a transmission electron microscope with resonances resembling the well-known standingwave mechanical oscillations on a restricted string. Overall, this picture consolidates the classical understanding of sub-wavelength plasmon wave phenomena. Along with the tremendous advances not only in fabrication [99], including lithography for top-down nanopatterning of metals, but also the more bottom-up chemical synthesis of complex metallic nanoparticles, the ability to experimentally explore even smaller length scales has generated also a renewed theoretical interest in $\sigma\left(\boldsymbol{r}, \boldsymbol{r}^{\prime}\right)$ for the opticalfrequency nonlocal response of plasmonic nanostructures. In the following, we offer a further introduction to this.

\subsubsection{Why be concerned with the nonlocal response?}

The LRA seems to be inherent to our general understanding and use of linear-response theory, and it is boldly taken for granted by the vast majority of introductory textbooks in the general field of electromagnetism and even beyond. The obvious way to mathematically connect Eqs. (4) and (2) is through the introduction of the Dirac delta function $\delta(\boldsymbol{r})$, having the usual property that $\int \mathrm{d} \boldsymbol{r}^{\prime} \delta\left(\boldsymbol{r}-\boldsymbol{r}^{\prime}\right) \mathcal{F}\left(\boldsymbol{r}^{\prime}\right)=\mathcal{F}(\boldsymbol{r})$ for a general function $\mathcal{F}$. As such, the Dirac delta function representations

$$
\begin{gathered}
\varepsilon\left(\boldsymbol{r}, \boldsymbol{r}^{\prime}, \omega\right) \approx \delta\left(\boldsymbol{r}-\boldsymbol{r}^{\prime}\right) \varepsilon(\boldsymbol{r}, \omega) \\
\sigma\left(\boldsymbol{r}, \boldsymbol{r}^{\prime}, \omega\right) \approx \delta\left(\boldsymbol{r}-\boldsymbol{r}^{\prime}\right) \sigma(\boldsymbol{r}, \omega)
\end{gathered}
$$

convey the short-range assumption behind the LRA, while also hinting that the LRA could be phenomenologically relaxed by substituting the Dirac delta function with a finite-range function, such as a Gaussian-type response function [100]. This will be the topic of later discussions in Section 2.1.

As already touched upon, the rationale behind the LRA is that one only has a material response in the same point of space as where the perturbation of the material is being applied. This may on the one hand feel intuitive and in accordance with the often absence of long-range correlation. On the other hand, if one imagines a compressible jelly soup of matter, then one can clearly also imagine some finite-range response of the substance when applying a local force. When treated quantum mechanically, the electron gas constitutes a compressible gas with a finiterange response governed by intrinsic length scales of the electron gas. For typical metals, the Fermi wavelength is of 
the order $\lambda_{\mathrm{F}} \sim 0.5 \mathrm{~nm}$ [86], thus suggesting a quite shortrange response. At the same time, the perturbation might not be entirely local or point-like in space. As we shall discuss, both of these issues matter for the validity of the LRA in metals. The discussion of this will, however, take a more formal starting point.

\subsubsection{Symmetries and conservation laws}

Symmetries are ubiquitous in physics while being inherently connected to also conservation laws. This was already appreciated in the early 1900s by Noether [101] and it constitutes a cornerstone in our understanding of physical processes with a mutual exchange of energy and momentum of the involved degrees of freedom. Following Noether's theorem, the commonly accepted principle of conservation of energy conservation follows stringently from the timeinvariance of the system. In the present context, spatial translational invariance immediately implies the conservation of momentum. Thus, to better appreciate the strength of light-matter interactions and the possible mutual coupling of light and plasmons, we shall next consider the energy and momenta of both kind of excitations.

\subsubsection{The energy and momentum carried by photons}

Solving the Maxwell's equations in free space, i.e. $\rho(\boldsymbol{r}, \omega)$ $=\sigma(\boldsymbol{r}, \omega)=0, \varepsilon(\boldsymbol{r}, \omega)=\varepsilon_{0}$, and $\mu(\boldsymbol{r}, \omega)=\mu_{0}$, one arrives at the well-known dispersion relation of light, $\omega(\boldsymbol{k})=c k$, where $\boldsymbol{k}$ is the wave vector (of length $k=|\boldsymbol{k}|$ ) associated with the spatiotemporal harmonic oscillation of the plane-wave form $\mathrm{e}^{\mathrm{i}(\boldsymbol{k} \cdot \boldsymbol{r}-\omega t)}$. The corresponding momentum of the photons is $\hbar k=\hbar \omega / c$ and for optical energies, say, $\hbar \omega \sim 3 \mathrm{eV}$, this gives a wave vector in the order of $k \sim 3 \times 10^{-3} \mathrm{~nm}^{-1}$. Here, the unit has been chosen in the anticipation of light interacting with nanoscale structures. The bottom line is that this photon momentum is very small in magnitude in comparison with the characteristic momenta of any other degrees of freedom in light-matter interactions, thus justifying the common assumption that photons carry hardly any momentum themselves. In semiconductor optics [102], this is the reasoning behind the picture of vertical interband transitions (in an infinite, translational invariant semiconductor system), i.e. $\Delta \boldsymbol{q}=\boldsymbol{k} \approx 0$, where $\Delta \boldsymbol{q}$ is the change in wave vector associated with the excitation of an electron across the bandgap in the electron energy band structure $\mathcal{E}(\boldsymbol{\kappa})$. Here, the principle of energy conservation gives $\mathcal{E}_{c}\left(\boldsymbol{\kappa}^{\prime}\right)=\mathcal{E}_{v}(\boldsymbol{\kappa})+\hbar \omega(\boldsymbol{k})$, with the subscripts denoting the conduction and valence bands, while momentum conservation implies that the initial and final wave vectors are given by $\boldsymbol{\kappa}$ and $\boldsymbol{\kappa}^{\prime}=\boldsymbol{\kappa}+\Delta \boldsymbol{q} \approx \boldsymbol{\kappa}$, respectively. In summary, this leads to the common condition $\mathcal{E}_{c}(\boldsymbol{\kappa}) \approx \mathcal{E}_{v}(\boldsymbol{\kappa})+\hbar \omega$, with the wave-vector dependence of the photon energy being suppressed to highlight its negligence. In terms of the constitutive equations, this justifies the well-accepted approximation $\varepsilon(\boldsymbol{q}, \omega) \approx \varepsilon(\boldsymbol{q} \rightarrow 0, \omega)$, which is the Fourier-space version of the LRA. One argument why the LRA is so reasonable thus relates to the tiny momentum carried by the photon. Another take on this comes from a real-space look at the light field associated with the photon, oscillating in real space with a wavelength of $\lambda=2 \pi / k \sim 400 \mathrm{~nm}$. With the Abbe diffraction limit in mind [95], no matter the effort in focusing fields with such wavelength components, the perturbation of the matter will be far from point-like. The effect of spatially averaging a local perturbation over a finite region is to smear the nonlocality.

\subsubsection{The energy and momentum of surface plasmons}

Surface plasmons on planar interfaces are in-plane spatiotemporal harmonic oscillations of the plane-wave form $\mathrm{e}^{\mathrm{i}\left(\boldsymbol{q}_{\|} \cdot \boldsymbol{r}_{\|}-\omega t\right)}$, being tightly confined to the interface of the metal (with dielectric function $\varepsilon$ ) and the dielectric (with dielectric function $\varepsilon_{d}$ ). The common dispersion relation can be obtained analytically within the LRA $[66,67,103$, 104]

$$
q_{\|}(\omega)=\frac{\omega}{c} \sqrt{\frac{\varepsilon(\omega) \varepsilon_{d}(\omega)}{\varepsilon(\omega)+\varepsilon_{d}(\omega)}} .
$$

Considering for a metal-air interface $\left(\varepsilon_{d}=1\right)$ and invoking for simplicity the loss-less Drude model (Eq. (8) with $\gamma \rightarrow 0$ ) to mimic $\varepsilon(\omega)$ of the metal, the corresponding dispersion relation

$$
q_{\|}(\omega)=\frac{\omega}{c} \sqrt{\frac{\omega^{2}-\omega_{p}^{2}}{2 \omega^{2}-\omega_{p}^{2}}}
$$

exhibits a diverging- $q_{\|}$behavior when approaching $\omega$ $=\omega_{\mathrm{SPR}} \equiv \omega_{p} / \sqrt{2}$. This is known as the surface-plasmon resonance (SPR) [66]. Let us now return to the discussion of the momentum carried by a surface-plasmon excitation and how it compares to the momentum carried by a photon of the very same energy.

The dispersion relation illustrated in Figure 2 has two branches - the polariton branch, which for low frequencies resembles the dispersion of photons, and the plasmon branch, where the wave vector diverges as one approaches the SPR. In the former case, the momentum consequently resembles that of a photon, while in the latter case, there is a large gap in momentum between the photon and the SP. Following the discussion on Noether's theorem, 


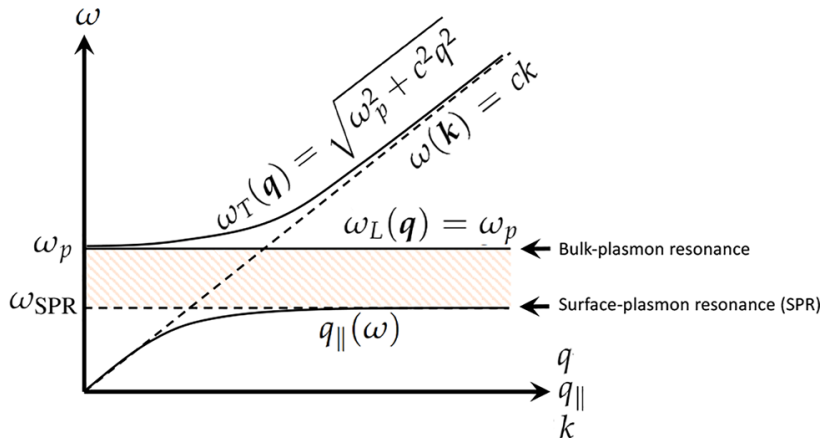

Figure 2: Surface-plasmon dispersion relation (Eq. (10b)), indicating also the dispersion of transverse waves above the plasma frequency, and the nondispersive (due to the local-response approximation) bulk plasmons at the plasma frequency. For comparison, the linear dispersion of photons in a vacuum is also shown (dashed).

this prohibits the plane-wave excitation of SPs on planar, translation invariant metal surfaces. Alternatively, if by some means SPs are excited, they will be nonradiative. The momentum gap is in some way both a blessing and a curse for the applications of SPs; they are conveniently confined to the surface, but they are also difficult to excite. Here, the key to optical excitation of SPs is to break the translation invariance, thus relaxing the requirement for momentum conservation. This can either occur in the excitation part (in principle through the use of spatiotemporal inhomogeneous fields [84]) or in the surface topography that is hosting the SPs, e.g. through the exploitation of nanostructured metal surfaces and metal nanoparticles of varying morphology. The above discussion, however, also highlights a more fundamental issue in the so-far classical treatment of SPs. The wave vector of the SP can exceed that of the corresponding photon, thus facilitating even shorter real-space oscillations of the corresponding electromagnetic fields. This has the intriguing consequence that SP can focus electromagnetic energy beyond Abbe's diffraction limit, with the important practical implication of bringing electromagnetic energy to the very nanoscale [84, 105-109]. On the one hand, the diverging and singular behavior [110] is accompanied by a corresponding enhancement of local electromagnetic fields, which has been fueling a lot of the interests in plasmonics. On the other hand, the diverging behavior is to some extent an artifact of the LRA and the negligence of plasmon damping. However, what is the limit then to this focusing, and how much of the diverging behavior remains in a more accurate description? As one approaches such singularities, the decreasing group velocity $\left(\partial \omega / \partial q_{\|}\right)$causes a slow-light enhanced susceptibility to damping [111], which in turn limits the slow-down itself [112-114]. In the case of the SPR, Ohmic damping would smear the diverging behavior $[66,115]$. However, even in the hypothetical absence of such damping [116], the plasma oscillations cannot physically occur on shorter length scales than those supported by the underlying electron gas itself. This is where the quantum nature of the electron gas comes into play, essentially introducing a cut-off as one approaches $\omega / v_{\mathrm{F}}[104,117]$.

\subsubsection{The energy of localized surface-plasmon resonances}

In the initial discussion of SPs, we considered translational invariant systems in the context of the SP dispersion relation, i.e. the wave vector $q_{\|}(\omega)$ and its energy dispersion (Eq. (10a)). Turning now the attention to structures of a finite extension, this wave vector is being quantized, in turn causing discrete values of the associated frequency. As a result, we will have localized surface-plasmon resonances (LSPR). An alternative perspective on this starts from the initial discussion of the Drude theory, where the negatively charged gas of free electrons was oscillating freely with respect to the positively charged background of the atoms in the metal. In the equation of motion (Eq. (7)), this was resembled by the absence of any restoring force. Turning now the attention to metallic domains of a finite extension, e.g. embedded in vacuum or some dielectric, the displacement of the electron gas with respect to the positively charged background will naturally involve a restoring force. In equilibrium, there is overall no net charge on the domain. Obviously, the numbers of electrons and equally many positively charged atoms are conserved even when the electron gas is slightly displaced away from its equilibrium position. However, on the one edge of the domain, there will locally be a slight excess of electrons, while this is balanced by a slight deficit of electrons on the opposite edge. ${ }^{1}$ Coulomb forces between those regions of induced charge densities (of opposing sign) will naturally work to restore the equilibrium. As such, the system now behaves like a mass-spring system and it will exhibit a natural frequency. Including a restoring force in Eq. (7) leads to the Drude-Lorentz model.

1 Within the LRA, the electron gas is incompressible, thus being displaced as a rigid body. As a consequence, the induced charge is strictly of a surface kind, i.e. an induced surface charge density. In the later treatments, this will be relaxed by accounting for the finite compressibility of the quantum electron gas. 
To further appreciate the formation of the LSPR, we will for simplicity focus the discussion on an archetype problem in electromagnetism: the polarizability of a spherical particle in a homogeneous field. How can this problem be relevant to a plasmonic nanoparticle at optical frequencies? In the quasi-static regime, where the particle radius $R$ is much smaller than the wavelength $\lambda$ of the polarizing field, this does indeed to a first approximation resemble the above textbook problem, and in agreement with full treatment of also radiation effects within Mie theory [118, 119]. Also, the smaller the particle, the better is this quasi-static approximation (QSA).

In this way, the polarizability $\alpha(\omega)$ associated with the dipole resonance is in the QSA given by [66]

$$
\alpha(\omega)=4 \pi R^{3} \frac{\varepsilon(\omega)-\varepsilon_{d}(\omega)}{\varepsilon(\omega)+2 \varepsilon_{d}(\omega)},
$$

which closely resembles the well-known ClausiusMossotti relation, or equivalently the Lorentz-Lorenz equation, that describes a homogeneous diluted mixture of such polarizable particles. For metals, with a negative $\varepsilon$, the polarizability can be resonantly enhanced at the frequency pole of this expression, defined by the Fröhlich condition

$$
\varepsilon^{\prime}(\omega)+2 \varepsilon_{d}^{\prime}(\omega)=0,
$$

involving the real parts of $\varepsilon=\varepsilon^{\prime}+\mathrm{i} \varepsilon^{\prime \prime}$ and $\varepsilon_{d}=\varepsilon_{d}^{\prime}+i \varepsilon_{d}^{\prime \prime}$. For a Drude metal in vacuum, it immediately gives $\omega_{\mathrm{LSPR}}$ $=\omega_{p} / \sqrt{3}$. Thus, the metallic particle supports a dipole resonance (and for that sake also high-order resonances [120]) below the plasma frequency. While the strength of the polarizability naturally depends on the size $R$ of the particle, intuitively giving smaller particles a smaller cross section, the resonance frequency is in itself sizeindependent within this QSA. In a quantum treatment of electrons-in-a-box, the electrons occupy discrete energy levels that reflect the size of the box [by quantizing the wave vector $\kappa$ of electrons and thus in turn $\mathcal{E}(\kappa)$ ], thus giving size-dependent optical features associated with single-particle transitions. On the other hand, the collective response of all the electrons (at near the Fermi level) is seemingly occurring at a size-independent frequency determined entirely by the Drude dielectric function. Adding to this, the resonance linewidth will be sizeindependent too. Indeed, this result is enabled by the nontrivial dispersion in Eq. (10b) in the large- $q_{\|}$limit.

The above discussion is relevant even beyond spherical particles since in the quasi-static limit there is no real dependence on the morphology of the particle. As such, the resonance properties mainly reflect the material properties captured by $\varepsilon(\omega)[121,122]$. This apparent paradox is a result of the LRA and the negligence of radiation effects inherent to the above treatment. As we shall see, relaxing the LRA to include quantum corrections associated with the finite compressibility of the electron gas, we indeed find $R^{-1}$ size corrections to the quasi-static result for both the LSPR frequency and its linewidth.

\subsubsection{Nanoscale experimental explorations}

The preceding sections have hopefully served to establish a curiosity for nonlocal effects in the optical response of plasmonic nanostructures. The aim of this section is to drive home the point by also providing the experimental arguments why explorations of quantum and nonlocal effects are both intriguing and timely problems in the field of plasmonics. In a way, the argument is two-fold, representing developments in both the ability of nanotechnology to not only enable true nanoscale plasmonic structures but also the advancements in instrumentation and measurement techniques, now facilitating the probing and resolution of plasmons and their hosting environments down to almost the atomic length scales [96, 97]. The experimental community now has access to both samples and experimental techniques that have enabled intriguing observations that seriously challenge the foundation and understanding of light-matter interactions inherently rooted in the common and widespread assumption of a local electrodynamic response of matter.

Figure 3 displays a selection of noble-metal-based plasmonic nanostructures from the international literature, being enabled with modern techniques in nanotechnology and nanofabrication, ranging from bottomup chemically synthesized metal nanoparticles to topdown patterning of metals by electron-beam lithography (EBL) and focused-ion beam (FIB) lithography. The examples have the exhibition of extreme nanoscale features in common, while also serving to highlight the capabilities of scanning transmission electron microscopy (STEM) in imaging these nanostructures with a resolution reaching the atomic scale. As an example, panel (A) shows a nanoparticle dimer formed by slightly elongated chemically grown crystalline gold nanoparticles separated by a $1 \mathrm{~nm}$ gap, while the STEM zoom-in image illustrates the presence of the atomic columns in the crystalline particles and the atomic-scale perfect ordering of the particles' surfaces. Likewise, panel (B) shows a chemically synthesized $5 \mathrm{~nm}$ silver nanoparticle with the STEM enabling the visualization of the atomic columns in the crystalline particle. Panel (C) illustrates the ability of FIB to make steep and narrow nanogrooves on a gold surface. Here, the deep 

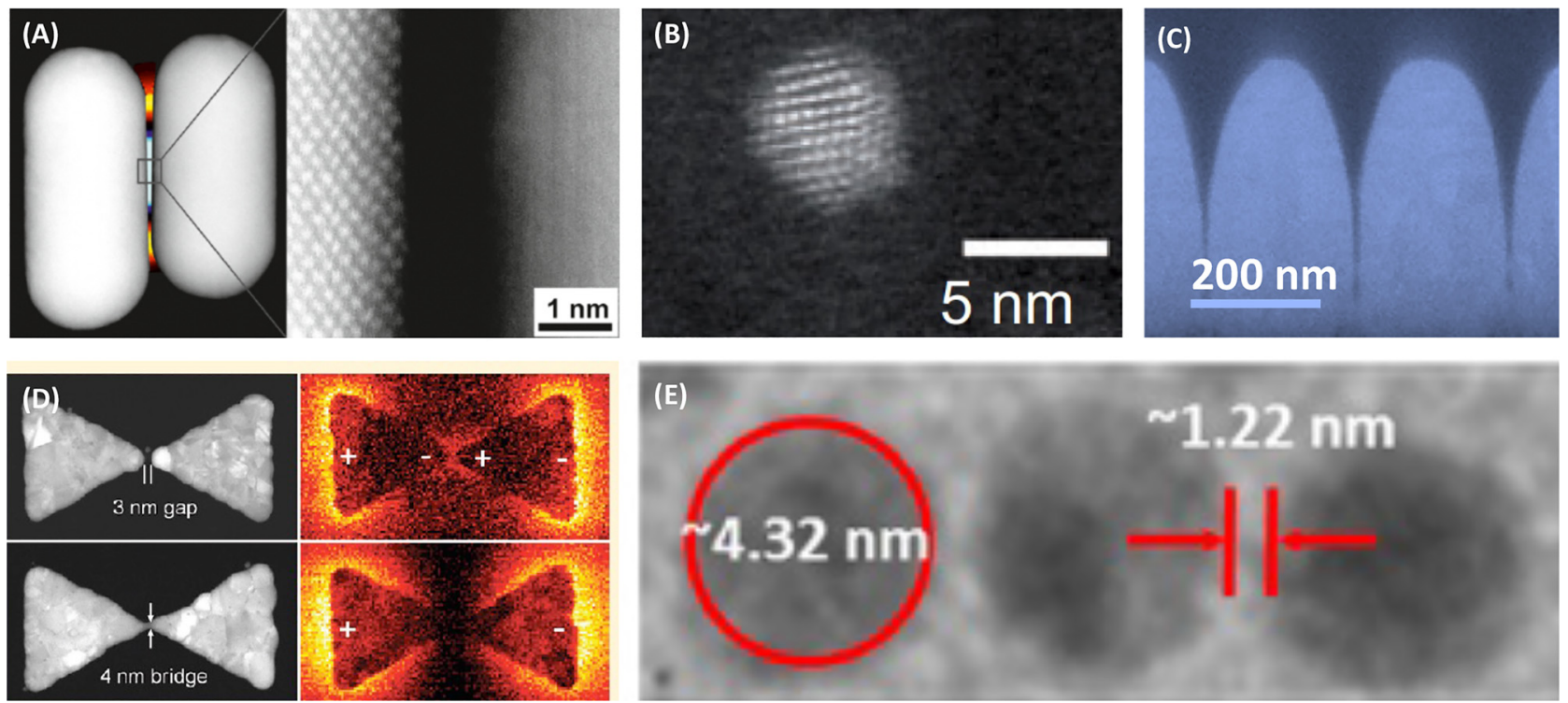

Figure 3: Selection of scanning transmission electron microscopy images of plasmonic nanostructures, all with a common feature of exhibiting true nanoscale features. (A) Gold nanoparticle dimer exposing a nanogap (left) and with clear indications of the atomic columns (right). Figure adapted from Ref. [123] (C) 2012 American Chemical Society). (B) Silver nanoparticles with a clear indication of the atomic columns. Figure adapted from Ref. [124]. (C) Steep and narrow nanogrooves in the gold surface. Figure adapted from Ref. [125]. (D) Gold bow-tie antennas with nanogaps. Figure adapted from Ref. [126] (C) 2012 American Chemical Society). (E) An array of gold nanoparticles with nanogaps. Figure adapted from Ref. [127] (C) 2017 American Chemical Society).

trenches constitute metal-insulator-metal (MIM) structures [128] with the opposing metal surfaces being separated by nanogaps in the few-nanometer regime. Panel (D) illustrates the STEM imaging of gold bow-tie antennas with the formation of 3-4 $\mathrm{nm}$ gaps enabled by EBL. The right-hand sub-panel displays the hybridized plasmon modes probed and visualized with EELS. Finally, panel (E) shows STEM imaging of superlattices of chemically synthesized gold nanoparticles, being themselves in the sub-5 nm regime, while separated from neighboring particles by even smaller $\sim 1 \mathrm{~nm}$ gaps. These tiny gaps of plasmonic dimers can support tremendously enhanced local fields (see e.g. tutorial explanation in the supplementary information of Ref. [129]), and the concept has been explored for plasmon-enhanced light-matter interactions [130-133] with astonishing field-enhancement factors.

The impressive capabilities of nanofabrication and nanoscale imaging can be breathtaking on their own, but the next challenge to digest is how to theoretically account for the light-matter interactions in such systems. Here, the majority of interpretations rely on the use of Ohm's law and the Drude model (Eqs. (2c) and (8)) which fully neglect the existence of mesoscopic phenomena, quantum effects, and the existence of atomic-scale details in the structures.
The overall hypothesis underlining the formalism reviewed is that as the characteristic length scale of metallic nanostructures $a$ start to become comparable to the nonlocal length scale $\xi$ of the jelly electron gas, nonlocal corrections to the LRA are anticipated. Nevertheless, the LRA procedure was endorsed in the analyses of experimental data in Ref. [126] from the group of Joel Yang. The title of this early seminal paper quite concisely summarizes the somewhat surprising conclusion [126]: "Nanoplasmonics: Classical down to the Nanometer Scale”. Having digested the implications of this paper, one may seriously question the entire motivation for the efforts to establish a mesoscopic electrodynamics framework. However, the apparent consequence of this seminal work has been further challenged by a number of later works (partly stimulated by Ref. [126]), now consolidating the consensus that quantum and nonlocal corrections to the LRA are critical to the interpretation of experiments.

The particle-on-a-mirror configuration was experimentally enabled by the hybridization of gold nanoparticles and substrates with the nanogap being enabled by the use of ultra-thin spacer materials [134]. As the gap is reduced, one observes an increasing redshifting, in accordance with the expectations based on the LRA and the use of plasmonic hybridization theory [135]. However, 
for gaps smaller than 1-2 $\mathrm{nm}$, the resonance is significantly blueshifted with respect to the anticipations from the LRA. The blueshifting is quite appreciable, significantly exceeding the error bars and resolutions of the optical spectroscopy, while eventually constituting more than $\Delta \lambda_{\text {LSPR }} \sim 100 \mathrm{~nm}$ for the smallest gap. This is an observation that cannot immediately be accounted for by the LRA, and the authors suggest a nonlocal origin of the blueshifting, being promoted by the very small gap.

In particle-on-a-substrate configurations, independent works [136-139] have been addressing the dipole resonance in the particles with nonlocal effects being promoted by the smallness of the particles themselves. With reference to the $R^{3}$ prefactor in the polarizability (Eq. (11a)), the particles are too small to have a significant optical cross-section [140]. Instead, they are being explored with EELS, while simultaneously exploiting STEM to image the particles, which allows a unique correlation of plasmon energies with the particle sizes. Despite significant error bars associated with the determination of both the $\omega_{\text {LSPR }}$ and $R$, there is an unambiguous and significant blueshift by up to $\Delta \omega_{\mathrm{LSPR}} \sim 0.5 \mathrm{eV}$ for the smallest particles. This cannot be accounted for by the LRA, which anticipates that $\omega_{\mathrm{LSPR}}=\omega_{p} / \sqrt{3}$ is independently of the radius. Instead, a nonlocal origin of blueshifting has been proposed, even though the experimental blueshifting [138] appears to exceed the theoretical expectation [141]. Independent observations of similar results were used to promote an alternative electron-in-a-box interpretation [139], where the collective plasmon oscillation would be explained as a result of many individual electron transitions between occupied and unoccupied electron energy levels in the metal particle [142]. The interpretation of Ref. [139] was later debated [143], and essentially, the radius $R$ seems too large compared to the Fermi wavelength $\lambda_{\mathrm{F}}$ to cause appreciable and resolvable quantized energy levels of the electronic structure in the room-temperature experiments [144]. However, irrespective of the interpretations, the independent and experimentally sound observations of blueshifts in Refs. [136-139] stand as a result that cannot be appreciated within the LRA.

As a final example of a much longer-standing experimental observation, optical spectroscopy was done on silver nanoparticles embedded in different gases [145]. In the case of an argon host medium, the experiments illustrate very clear $R^{-1}$ size-dependent corrections in both the frequency position and the resonance linewidth. This has as well been addressed by Kreibig and co-workers $[146,147]$, including also a more comprehensive treatment
[148]. In passing, we note that this far-field measurement is robust to inhomogeneous broadening associated with a possible size-dispersion in the ensemble of measured particles [149]. The size-dependent broadening is now a well-accepted phenomenon being accounted for by pragmatically adding a so-called Kreibig term to the bulk damping [148], i.e. $\gamma \rightarrow \gamma+A v_{\mathrm{F}} / R$, where empirically $A \sim 1$. Microscopically, the Kreibig damping can be understood in the context of surface-enhanced Landau damping [150-154] manifesting only at the surface, and due to the surface-to-volume ratio $\left(S / V \propto R^{-1}\right)$ this eventually leads to an $R^{-1}$ correction. However, for three decades its more explicit relation to the nonlocal response has perhaps been less clear and there has been no explicit and unified way of accounting for size-dependent frequency shifts and broadening in the electrodynamics of more arbitrarily shaped geometries. As will be discussed, the mesoscopic electrodynamic framework fills this gap.

At this stage, it is instructive to briefly touch on the Landau damping of plasmons [155]. Intuitively, plasmons cannot oscillate at shorter length scales (larger wave vectors) than allowed for by the Fermi properties of the underlying electron gas itself. The microscopic mechanism that prevents this from manifesting is known as Landau damping [155], which suppresses plasmon excitation in metal with a wave vector exceeding $\omega / v_{\mathrm{F}}$ (with $v_{\mathrm{F}}$ being the Fermi velocity), in which case plasmons decay into electron-hole pairs [51]. In plasmonics, the bulk Landau damping is perhaps not of too much concern, while its influence can be significantly enhanced near surfaces [116]. Here, the abrupt change in confinement supports large Fourier components of the electromagnetic fields, which exceed the unset for Landau damping, thus leading to a surface-enhancement of Landau damping [154, 156-158]. Landau damping will be discussed in more detail in Section 7.3.

In passing, plasmonic dimers sub-nanometric gaps have been subject to intense experimental research [123, 126, 139, 159-163]. Promoting extreme light-matter interactions [109], they have been anticipated to host quantumtunneling mediated charge-transfer plasmons (CTP) [82, 164], and there have been experiments claiming optical-frequency quantum tunneling phenomena in plasmonic dimers [139, 159]. Clearly, the theoretical explanation of such phenomena would require formalism beyond the LRA. As will be discussed, the mesoscopic electrodynamics being reviewed challenges the importance of tunneling effects as a significant source of dissipation in metal-based plasmonic dimers, drawing instead attention to quantum corrections associated with surface-enhanced Landau damping and surface-mediated scattering. 
The above discussion has independently emphasized nanoparticle monomers and dimers, while perhaps leaving the impression that their electrodynamics represent different and independent physics. Indeed, the literature is often alluding to either quantum-size (monomers) or quantumtunneling effects (dimers). In closing this introduction, the reviewed mesoscopic electrodynamic formalism offers a unified description, advocating that for many experimentally accessible configurations the compressibility of the quantum electron gas and the surface-enhanced Landau scattering constitute the dominating and common way that quantum nonlocal response influences the electrodynamics of both monomers and dimers.

\section{Generalized nonlocal wave equation}

To aid a more general discussion of quantum corrections to the classical electrodynamics, this section starts from the general IDE formulation of nonlocal response in Eq. (5), here without loss of generality written with an emphasis on the convenient dielectric function representation

$$
\boldsymbol{\nabla} \times \boldsymbol{\nabla} \times \boldsymbol{E}(\boldsymbol{r})=\left(\frac{\omega}{c}\right)^{2} \int \mathrm{d} \boldsymbol{r}^{\prime} \varepsilon\left(\boldsymbol{r}, \boldsymbol{r}^{\prime}\right) \boldsymbol{E}\left(\boldsymbol{r}^{\prime}\right) .
$$

While this IDE form clearly conveys the nonlocal nature of the problem, we will here pursue the goal of a fully equivalent PDE formulation involving only a single PDE for the electrical field. In particular, Ref. [165] posed the curious question if nonlocal electrodynamics could be reformulated in terms of the following second-order PDE?

$$
\boldsymbol{\nabla} \times \boldsymbol{\nabla} \times \boldsymbol{E}(\boldsymbol{r})=\left(\frac{\omega}{c}\right)^{2}\left[\varepsilon(\omega)+\xi^{2} \mathbb{W}\right] \boldsymbol{E}(\boldsymbol{r}) .
$$

If so, we would have rendered the problem mathematically local in nature, i.e. only involving one spatial coordinate. Furthermore, we would also have eliminated the $\boldsymbol{J}$-field problem, leaving us with a generalized eigenvalue problem involving only the $\boldsymbol{E}$-field. Conceptually, this would be no small feat, being also of practical importance for computational electrodynamics [166] with accounts for the nonlocal plasmonic response. At this stage, $\mathbb{W}$ remains an abstract second-order linear partial-differential operator representing the nonlocality and its correction to the LRA Drude dielectric function $\varepsilon(\omega)$, while $\xi$ would be a length scale being characteristic of the finite range of the nonlocal response. Below, we discuss possible embodiments of the operator in more detail.

\subsection{Short-range isotropic response}

Inspired by the phenomenological approach of Ref. [100], let us focus on an isotropic homogeneous electron gas where $\varepsilon\left(\boldsymbol{r}, \boldsymbol{r}^{\prime}\right)=\varepsilon\left(\left|\boldsymbol{r}-\boldsymbol{r}^{\prime}\right|\right)$. We aim to relax the assumption of the local response in Eq. (9) by relaxing the Dirac delta function response to occur over a finite range. Anticipating that this range is not too long (consistent with $q^{2}$ wavevector corrections to the LRA Drude function [167]), we next introduce the first three moments of the shortrange isotropic response function

$$
\begin{gathered}
\int \mathrm{d} \boldsymbol{R} \varepsilon(|\boldsymbol{R}|)=\varepsilon(\omega), \\
\int \mathrm{d} \boldsymbol{R} \boldsymbol{R} \varepsilon(|\boldsymbol{R}|)=0, \\
\int \mathrm{d} \boldsymbol{R} \boldsymbol{R}^{2} \varepsilon(|\boldsymbol{R}|) \equiv \xi^{2} .
\end{gathered}
$$

Recalling Eq. (9), the (zeroth) moment of the response function naturally gives the Drude dielectric function $\varepsilon(\omega)$ associated with the LRA. The first moment vanishes for symmetry reasons and irrespectively of the details of the response function, since the integral involves the product of an even and an odd function, making the full integrand an odd function with respect to $\boldsymbol{R}=\boldsymbol{r}-\boldsymbol{r}^{\prime}$. The second moment by definition naturally gives the range $\xi$ of the response function. In addition to the systematic introduction of the range $\xi$, the usefulness of these moments will soon become clear. Finally, the $\xi$-notation is deliberately chosen to underline its close resemblance to the $\xi$ nonlocal length scale that will be introduced in proceeding sections.

\subsection{Transformation to PDE formulation}

Naturally, $\xi$ is a small length scale that must be associated with the intrinsic length scales of the electron gas. For the moment, one could have the Fermi wavelength $\lambda_{\mathrm{F}}$ in mind, or alternatively the Thomas-Fermi screening length $q_{\mathrm{TF}}^{-1}$ [86]. Even without knowledge about the microscopic details of the response function, it thus feels safe to assume that all other fields vary slowly on the length scale of $\xi$. In particular, as suggested in Figure 4, the $\boldsymbol{E}$-field inside the integral in Eq. (12) will vary on the much longer length scale of $\lambda$, which motivates a Taylor expansion of $\boldsymbol{E}\left(\boldsymbol{r}^{\prime}, \omega\right)$ around the point $\boldsymbol{r}$, anticipating that we can then subsequently perform the integral term by term. To second-order in $\left(\boldsymbol{r}^{\prime}-\boldsymbol{r}\right)$ this expansion gives

$$
\begin{aligned}
E_{\alpha}\left(\boldsymbol{r}^{\prime}, \omega\right) & \simeq E_{\alpha}(\boldsymbol{r}, \omega)+\nabla E_{\alpha}(\boldsymbol{r}, \omega) \cdot\left(\boldsymbol{r}^{\prime}-\boldsymbol{r}\right) \\
+ & \frac{1}{2}\left(\boldsymbol{r}^{\prime}-\boldsymbol{r}\right)^{\mathrm{T}}\left[\boldsymbol{\nabla} \nabla E_{\alpha}\left(\boldsymbol{r}^{\prime}, \omega\right)\right]\left(\boldsymbol{r}^{\prime}-\boldsymbol{r}\right) \ldots
\end{aligned}
$$




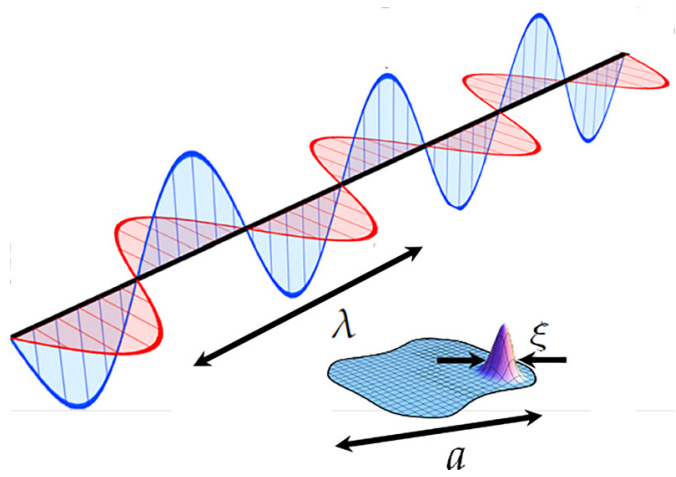

Figure 4: Schematic illustration of a short-range isotropic response function $\varepsilon\left(\left|r-r^{\prime}\right|\right)$ and the comparison of the nonlocal range $\xi$ to the dimension of the nanostructure $a$, and the wavelength $\lambda$ of the polarizing optical field. The analysis assumes that $\xi \ll a \ll \lambda$.

where the Hessian $\nabla \nabla$ has elements $\{\nabla \nabla\}_{\alpha \eta}=\partial_{\alpha} \partial_{\eta}$ and $\alpha=\{x, y, z\}$ and $\eta=\{x, y, z\}$. Here, we already start to see the contours of what the abstract operator $\mathbf{W}$ could possibly be.

When now performing the integral term by term, the moments defined in Eq. (14) will come in handy and we immediately see that leading order in this expansion, $\mathbb{W}$ will involve second-order partial derivatives. In fact, in a scalar treatment for the electrical field, the Taylor expansion immediately gives $\mathbb{W} \rightarrow \nabla^{2}[165]$. Second-order off-diagonal terms, such as $\partial_{x} \partial_{y}$, do not contribute for the same symmetry reasons that make the first moment vanish. Considering the vectorial nature, we could naturally also imagine terms in $\mathbb{W}$ involving the double-curl operator $(\nabla \times \nabla \times)$ and the gradient-of-divergence operator $(\boldsymbol{\nabla}[\boldsymbol{\nabla} \cdot])$. In passing, we note how curl and divergence operators are already common to the PDE formulation of Maxwell's equations (Eq. (1)) and that the double-curl operator is already featured on the left-hand side in both the IDE (Eq. (12)) and PDE (Eq. (13)) formulations of the wave problem.

How general is the above treatment then? Since the only assumption is that we have a short-range isotropic response, the nonlocal correction term $\xi^{2} \mathbb{W}$ should hold irrespectively of the microscopic details underlining the response function [165]. As such, one could even imagine several co-existing mechanisms contributing in concert to give a common overall nonlocal range of the response. As such, it seems natural to anticipate that $\xi^{2}=\sum_{i} \xi_{i}^{2}$. In Section 4 we shall explicitly consider one such example where convection and diffusion coexist to form exactly such a problem with $\xi^{2}=\xi_{\text {conv }}^{2}+\xi_{\text {dif }}^{2}[168]$.

With the formal formulation of the problem as a single PDE, it is now also apparent how nonlocal response effectively enters as a small smearing term, that blurs induced surface charge over a finite length scale. As such, it is also clear how quantum nonlocal effects tend to manifest as spectral shifts proportional to the surfaceto-volume ratio. Also, the LRA field singularities associated with abrupt variations in surface topology, commonly addressed through by-hand addition of artificial rounding [170], will now be smeared over a finite length scale $\xi$. An alternative perspective is that even when plasmons are confined in arbitrarily sharp boxes, the cut-off in the wave vector (essentially $\xi^{-1} \sim \omega / v_{\mathrm{F}}$ ) limits the higher Fourier components and consequently prevents a full resolving of the geometry. Figure 5 shows an example, where the metal surface has an abruptly varying surface topography. In the particular example, we show the electrical-field intensity distribution for the excitation of an arbitrarily sharp metallic tip. Clearly, the underlying geometry has a self-similarity, thus posing a singular problem for wave equations without any intrinsic length scales [171]. In the LRA such a structure would exhibit a strong field singularity [110] whereas nonlocal response serves to smear the induced charges and the field intensity over a characteristic length scale given by $\xi$. This brings us to also note a more practical side to the Laplacian-like diffusion term. As it is already well known from efforts to numerically solve the Navier-Stokes equation for hydrodynamic problems, the pragmatic addition of a small artificial diffusion is often useful and even essential to stabilize numerical solutions [172]. Here, the Laplacian-like term actually benefits numerical solutions of plasmonic problems in a similar

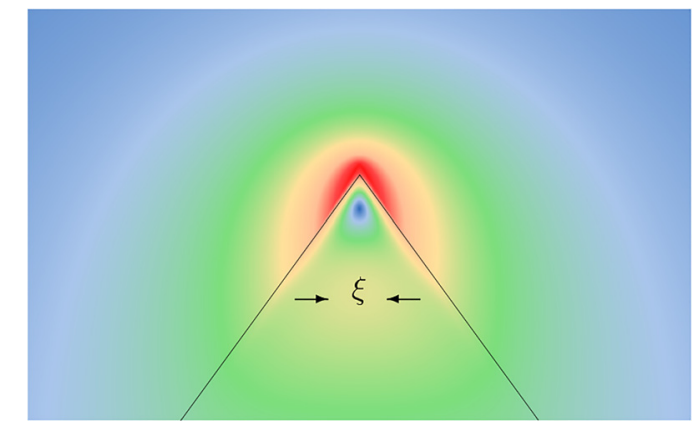

Figure 5: Typical electrical-field intensity for the plane-wave electromagnetic excitation of an arbitrarily sharp metallic tip. The nonlocal response serves to smear out the diverging field of the otherwise singular geometry. The results are obtained with the aid of a finite-element method implementation of the hydrodynamic model. Figure adapted from Ref. [169] (C) 2012 AIP Publishing). 
way, even though we shall in the next sections emphasize the physical origin rather than being an artificially introduced term. With this smearing term in the equation, electrodynamic simulations of metal structures with even arbitrarily sharp features in the surface topography will not suffer the usual convergence issues due to the underlying singular response [173].

As another example of the influence of a finite $\xi$, the hydrodynamic model has been applied to hyperbolic metamaterials [21] comprised alternating thin parallel layers of metal and dielectric. Here, the cut-off in plasmon wave vector $\xi^{-1} \sim \omega / v_{\mathrm{F}}$ serves to regularize the otherwise diverging density-of-states [174], thus imposing fundamental limitations on the enhancement of light-matter interactions that hyperbolic metamaterials can offer, e.g. in combination with quantum emitters. Similar limitations appear in relation to light-matter interactions enhanced in the near vicinity of nanocorrugated metal surfaces. As an example, the surfaced-enhanced Raman scattering [175-178] on corrugated surfaces [179] is naturally reduced by geometrical rounding [180], while nonlocal response poses further fundamental limitations on the otherwise diverging effect [181]. Having discussed the overall nonlocal modifications of the electrodynamic wave equation, we now proceed to specific semiclassical models that allow us to estimate the nonlocal length scale $\xi$, i.e. the strength of the quantum corrections to the LRA description.

\section{Semiclassical nonlocal hydrodynamic model - convection dynamics}

Having discussed the more general properties of nonlocal electrodynamics, we now proceed to a particular semiclassical embodiment of this. The nonlocal hydrodynamic treatment of electrons in metals dates back to seminal work by Bloch [49], and the later literature is rich, including the more comprehensive textbook by Boardman [182] and reviews by Barton [76] and later Pitarke et al. [60] and Raza et al. [183].

The starting point for the inclusion of nonlocal effects is in direct line with the Drude equation of motion that we have already considered in the introduction. However, rather than considering first the equation of motion for a single electron, while later multiplying by the density of electrons in quantum electron gas, we this time immediately adapt a fluidic picture that accounts also for a finite compressibility of the electron system. The hydrodynamic equation of motion is then given by (suppressing for transparency the spatial and temporal arguments)

$$
\overbrace{m\left[\partial_{t}+\boldsymbol{v} \cdot \boldsymbol{\nabla}\right] \boldsymbol{v}=-m \gamma \boldsymbol{v}-e \boldsymbol{E}}^{\text {Classical equation-of-motion }}-\overbrace{\boldsymbol{\nabla} \frac{\delta G[n]}{\delta n}}^{\begin{array}{c}
\text { Quantum } \\
\text { correction }
\end{array}}
$$

Here, $\boldsymbol{v}$ is now to be understood as the velocity field of the electron fluid. As in the Navier-Stokes equation of fluid dynamics, the acceleration term on the left-hand side is now emphasizing a Lagrangian specification with the usual time derivative replaced by the material (substantial) time derivative. On the right-hand side, we have the sum of forces acting on the fluid particle. In addition to the electrical part of the Lorentz force and the phenomenological damping term, we have compared to Eq. (7) now also included a gradient term of an energy-density functional associated with the density-dependent total energy of the electron gas. It is this pressure-like term that introduces the quantum mechanics of the electron system into the semiclassical equation of motion.

\subsection{Quantum description of the compressible electron gas}

Depending on the level of details that we consider in the quantum description of the electron gas, this energy functional can in addition to the internal kinetic energy of the electron gas also include correlation and exchange phenomena. For the free-electron gas with a parabolic dispersion relation it is relatively straightforward to show that $G \propto n^{5 / 3}$, and within Thomas-Fermi theory one likewise finds the result [86]

$$
G[n]=\frac{3 h^{2}}{10 m}\left(\frac{3}{8 \pi}\right)^{2 / 3} \int \mathrm{d} \boldsymbol{r} n^{5 / 3}(\boldsymbol{r}, t)
$$

and consequently the functional derivative becomes $\delta G / \delta n \propto m v_{F}^{2}\left(n / n_{0}\right)^{2 / 3}$, with $v_{\mathrm{F}}$ being the Fermi velocity associated with the equilibrium electron density $n_{0}$ [86]. Note how the equation of motion in Eq. (16) is both nonlinear and nonlocal, thus forming a starting point for also nonlinear and nonlocal plasmonics [184-186], while we will here aim for a linear relationship between the induced current density $\boldsymbol{J}$ and the driving $\boldsymbol{E}$-field.

\subsection{Linear-response nonlocal constitutive relation - quantum corrections to Ohm's local-response law}

In the efforts to extract the linear-response part of Eq. (16) and determine the frequency-domain generalized constitutive law, we first differentiate the equation of motion with 
respect to time and then invoke the continuity equation,

$$
\partial_{t} n=-\nabla \cdot(n \boldsymbol{v})
$$

to eliminate $\partial_{t} n$. In the spirit of linear-response theory, the equation of motion is linearized, exploiting the following linearization procedure [183]

$$
\begin{gathered}
n(\boldsymbol{r}, t)=n_{0}+n_{1}(\boldsymbol{r}, t), \\
\boldsymbol{v}(\boldsymbol{r}, t)=\boldsymbol{v}_{1}(\boldsymbol{r}, t), \\
\boldsymbol{E}(\boldsymbol{r}, t)=\boldsymbol{E}_{1}(\boldsymbol{r}, t), \\
\frac{\delta G[n]}{\delta n}=\left(\frac{\delta G[n]}{\delta n}\right)_{0}+\left(\frac{\delta G[n]}{\delta n}\right)_{1} .
\end{gathered}
$$

As a result, $v$ is the velocity correction to the static sea of electrons with a homogeneous equilibrium density $n_{0}$, while $n_{1}$ is likewise the small $\left(n_{1} \ll n_{0}\right)$ induced density variation associated with the driving $\boldsymbol{E}$-field. We emphasize that with the assumption of a homogeneous equilibrium density $n_{0}$, we are naturally excluding any quantumconfinement effects associated with a finite size $a$ of the metallic domains, which we may safely do as long as we have $a \gg \lambda_{\mathrm{F}}$. Furthermore, we are also implicitly neglecting inhomogeneity in the near vicinity of the metal surface [187], such as Friedel oscillations [188] on the length scale of the Thomas-Fermi screening length $q_{\mathrm{TF}}^{-1}$. On a similar footing, we are also neglecting the possible quantum spill-out associated with a finite work function and the evanescent quantum electron wave functions into the classically prohibited vacuum surrounding the metal domain. On the other hand, $n_{1}(\boldsymbol{r}, t)$ is allowed to be inhomogeneous, and indeed it will be so in the vicinity of the metal surface. Clearly, these chosen physical assumptions will be reflected in the boundary conditions that we will return to in Section 3.3.

Finally, the linearization procedure is completed by a Fourier transformation to the frequency domain to give the following coupled PDE problem, constituting the master equation for the nonlocal electrodynamics [189]:

$$
\begin{array}{r}
\boldsymbol{\nabla} \times \boldsymbol{\nabla} \times \boldsymbol{E}(\boldsymbol{r})=\left(\frac{\omega}{c}\right)^{2} \boldsymbol{E}(\boldsymbol{r})+i \omega \mu_{0} \boldsymbol{J}(\boldsymbol{r}), \\
\underbrace{\xi^{2}(\omega) \boldsymbol{\nabla}[\boldsymbol{\nabla} \cdot \boldsymbol{J}(\boldsymbol{r})]}_{\text {Quantum correction }}+\underbrace{\boldsymbol{J}(\boldsymbol{r})=\sigma(\omega) \boldsymbol{E}(\boldsymbol{r})}_{\text {Ohm'slaw }},
\end{array}
$$

where the dispersive strength of the quantum correction is given by

$$
\xi^{2}(\omega)=\frac{\beta^{2}}{\omega(\omega+i \gamma)}, \quad \beta^{2}=\frac{3}{5} v_{F}^{2}
$$

While Planck's constant does not appear in this expression, it is nevertheless a quantum correction since the Fermi velocity $v_{\mathrm{F}}$ is a concept unique to the quantum treatment of the electron gas [86]. To aid a transparent comparison to the LRA Drude problem, we have in Eq. (20a) deliberately omitted interband contributions $\left[\varepsilon_{\text {int }}(\omega)=1\right]$ in the first term on the right-hand side, thus fully resembling the omission of interband contributions in Eq. (8). Here, $\sigma(\omega)$ is the already introduced Drude conductivity within the LRA (Eq. (8)). We have considered the $\beta$-parameter appropriate for a three-dimensional (3D) electron gas, being reflected by the $3 / 5$ prefactor. For more details on this, we refer to work by Halevi [190] and the discussions by Raza et al. [183].

Clearly, Eq. (20b) constitutes a generalized constitutive equation, with the first term on the left-hand side containing the nonlocal quantum corrections to Ohm's law. The range $\xi \sim v_{\mathrm{F}} / \omega$ of the nonlocal response has a straightforward interpretation: it is approximately the distance that an electron would move by convection during the time of an optical cycle. For the noble metals, this distance is at most of the order of $1 \mathrm{~nm}$.

In terms of resonances, it can already be anticipated that this nonlocal correction term will be responsible for the previously discussed frequency shifts of resonances, i.e. $\Delta \omega_{\mathrm{LSPR}}$. Since $\gamma \ll \omega$, $\xi^{2}$ has only a small imaginary part, but in principle, this would add a small nonlocal broadening too. We shall later in Section 4 return to this.

\subsection{Boundary conditions}

When interfacing materials with different electromagnetic properties, boundary conditions constitute a central element of the electrodynamic problem [14]. As such, boundary conditions are also central to the nonlocal electrodynamics as first discussed by Pekar in the context of exciton problems [191]. For plasmons, this is perhaps also something that has caused some, at least initially, confusion in the literature [192, 193]. In the following, we summarize the considerations for the nonlocal hydrodynamic model.

In the LRA, the $\boldsymbol{J}$-field may be entirely eliminated from the problem, leaving us with a closed PDE for the $\boldsymbol{E}$-field. When subsequently looking for solutions inside a finite metallic domain, possibly coupled to the surrounding exterior vacuum, the PDE naturally needs to be solved subject to boundary conditions that will connect field solutions in the interior with those of the exterior. For the LRA problem, this reduces to the usual boundary conditions for the $\boldsymbol{E}$ field itself [14]. The boundary condition for $\boldsymbol{E}$ of course follows as a consequence of Maxwell's equations them- 
selves, i.e. the derivation of the boundary conditions only involves Maxwell's equations and the use of Gauss' and Stokes' theorems.

Turning to the nonlocal problem and the coupled PDE in Eq. (20), it is, however, immediately clear that now we also need an additional boundary condition (ABC) for the $\boldsymbol{J}$-field. While the literature might occasionally leave the impression of some arbitrariness and that there is some freedom in choosing the $\mathrm{ABC}$, the unambiguous message to be echoed in this review - which will be substantiated below - is that there is no such freedom for just convenient mathematical or numerical choices. In fact, once the physical context has been specified, the $A B C$ follows explicitly from already existing boundary conditions and the fulfillment of the continuity equation for the induced charge fluctuations. In other words, we should first formulate the physical assumptions and then boundary conditions simply follow from the governing equations. For more detailed accounts, we refer to Refs. [117, 168, 189, 194]. The above being said, one can of course imagine situations with surfaces of more complex matter, where unambiguous physical conditions are not easily formulated.

To proceed with the formulation of boundary conditions for the $\boldsymbol{J}$-field, the physical assumption is here to treat the electron gas as homogeneous (in the equilibrium), being confined by a hard-wall potential associated with a very high work function. In this way, $n_{0}$ is assumed constant throughout the metal while it drops abruptly to zero outside the metal surface. This formed a starting point for the specular-reflection model of Ritchie and Marusak [195] and the semiclassical infinite-barrier model of Fuchs and Kliewer [196]. The step-like variation unambiguously leads to exactly one required $\mathrm{ABC}$, namely the continuity of the normal component of the free-electron current density $\boldsymbol{J}[189,197]$. The implicit assumption of an infinite work function does not allow electrons outside the metal. Consequently, we have $\boldsymbol{J}=0$ outside the metal, while being still finite inside the metal. Thus, no electrons move across the metal surface, while they are still free to move parallel to the surface (in this way the hydrodynamics of electrons is different from the more common treatment of hydrodynamic flow assuming a no-slip Dirichlet-type boundary condition for the fluid velocity [198]). In other words, the normal component of $\boldsymbol{J}$ is zero at the surface, which we mathematically express as $\boldsymbol{n} \cdot \boldsymbol{J}=0$, with $\boldsymbol{n}$ being the normal vector. Here, we have emphasized the physical arguments, but this can also be derived rigorously by invoking Gauss' theorem [117].

\subsection{Reflections on physical assumptions}

Let us now briefly reflect on the physical assumptions. The assumption for the ground state of the electron gas corresponds to an infinite work function, thus suppressing quantum-spill out of electrons beyond the surface of the metal, while we are clearly also neglecting any density variations inside the metal, i.e. confinement effects such as Friedel oscillations [187]. The pragmatic defense is that despite the limitations, this picture is close in spirit to the LRA that involves an implicit assumption of a spatially uniform equilibrium electron density, i.e. $n_{0}(\boldsymbol{r}) \approx n_{0}$.

We emphasize that this is a reasonable first description of noble metals that are commonly employed in plasmonics, while spill-out effects are mainly important for less common cases like sodium [122, 199-202]. The effects of mutual interactions among the electrons depend naturally on $n_{0}$, and details of the equilibrium density profile are thus commonly explored for varying the Wigner-Seitz radius $r_{s} \propto n_{0}^{-1 / 3}$ [187], conveniently normalized by the Bohr radius $a_{0}$. For noble metals $r_{s}$ is roughly $2 a_{0}$ [86], while sodium is characterized by $r_{s} \simeq 4 a_{0}$, where the lower density reduces screening, thus causing more pronounced Friedel oscillations. In both cases, deviations from a uniform electron gas manifest on the Fermi wavelength scale, and only in the near vicinity of the surface.

For Friedel oscillations near the interior of the metal surface, we emphasize that there have been attempts of relaxing the assumption of a homogeneous equilibrium density [203, 203-206]. As an example, the self-consistent jellium results for $n_{0}(\boldsymbol{r})$ by Lang and Kohn [187] have been incorporated in LRA descriptions with a locally varying electron density [205]. Despite the intuitive simple extension of the LRA, this results in nonphysical singular behavior at points in space where $\varepsilon(\boldsymbol{r}, \omega) \sim 0$ (in the evanescent tail of the electron density), being an artifact of the underlying LRA [205]. The fact that the Fourier transform of $n_{0}(\boldsymbol{r})$ would introduce wave-vector components exceeding $\omega / v_{\mathrm{F}}$ (the onset of Landau damping) is resolved by self-consistent hydrodynamic treatments [207-209].

\subsection{Rewriting the coupled PDE problem}

Returning to Eq. (20) the next task is now to eliminate the $\boldsymbol{J}$-field from the problem, thus hopefully ending up with a single PDE for the $\boldsymbol{E}$-field. Here, the perhaps not so immediately obvious trick is to first operate on Eq. (20a) by taking the divergence. Since the divergence of any curl field is zero, we immediately get the general property that $\boldsymbol{\nabla} \cdot \boldsymbol{E} \propto \boldsymbol{\nabla} \cdot \boldsymbol{J}$. Applying this in turn to Eq. (20b) it straightforwardly allows us to now eliminate the $J$-field with the 
following result [194]

$$
\boldsymbol{\nabla} \times \boldsymbol{\nabla} \times \boldsymbol{E}(\boldsymbol{r})=\left(\frac{\omega}{c}\right)^{2}\left[\varepsilon(\omega)+\xi^{2}(\omega) \boldsymbol{\nabla}[\boldsymbol{\nabla} \cdot]\right] \boldsymbol{E}(\boldsymbol{r}) .
$$

It should be emphasized that this PDE has exactly the structure that we anticipated in Eq. (13), with $\boldsymbol{\nabla} \rightarrow \boldsymbol{\nabla}[\boldsymbol{\nabla} \cdot]$. We also emphasize that we have arrived at this exact result without invoking any approximations in the rewriting steps, and using only standard identities from the calculus of vector fields.

Regarding boundary conditions at the metal's surfaces, we note that in the context of Eq. (21), the surface boundary condition $\boldsymbol{n} \cdot \boldsymbol{J}$ implies that $\boldsymbol{n} \cdot \boldsymbol{E}$ is only continuous across the boundary in the absence of interband transitions and for vacuum surroundings. However, in general, there is a normal-component electric-field discontinuity if the interband contribution on the metal side is not fully compensated by dielectric contributions on the dielectric side of the interface [117].

To close the circle of the phenomenological arguments in Section 2.2, based on the Taylor expansion that led to a Laplacian nonlocal correction term, we use that for any vector field $\boldsymbol{F}$ we have $\boldsymbol{\nabla} \times \boldsymbol{\nabla} \times \boldsymbol{F}=\boldsymbol{\nabla}[\boldsymbol{\nabla} \cdot \boldsymbol{F}]-\boldsymbol{\nabla}^{2} \boldsymbol{F}$. In this way we can now introduce the Laplacian operator and grouping the additional double-curl term with the one already existing on the left-hand side, we arrive at the exact rewriting of the problem [194],

$$
\boldsymbol{\nabla} \times \boldsymbol{\nabla} \times \boldsymbol{E}(\boldsymbol{r})=\left(\frac{\omega}{c}\right)^{2} s(k \xi)\left[\varepsilon(\omega)+\xi^{2}(\omega) \nabla^{2}\right] \boldsymbol{E}(\boldsymbol{r}),
$$

where on the right-hand side $s(x)=\left(1-x^{2}\right)^{-1}=1+\mathcal{O}\left(x^{2}\right)$ gives a re-scaling of the initial eigenvalue $(\omega / c)^{2}$. For $k \xi=(\omega / c) \xi \ll 1$, being equivalent to $\xi \ll \lambda$, the re-scaling is, however, negligible and we finally arrive at the result in Eq. (13) with $\mathbb{W} \rightarrow \nabla^{2}$ already anticipated phenomenologically through the Taylor expansion procedure which indeed assumed as a starting point that $\xi \ll \lambda$.

Let us finally note that in relation to the differences between longitudinal and transverse polarization, the form of $\mathbb{W}$ will differentiate between the two polarizations. From the Fourier representation of the hydrodynamic model it is well-known that whereas the transverse component of the response function is wave vector independent, the longitudinal component depends on the wave vector [183]. Returning to Eq. (21), we indeed immediately find with a Helmholtz decomposition that transverse and longitudinal solutions satisfy the following

$$
0=\left[\varepsilon(\omega)\left(\frac{\omega}{c}\right)^{2}+\nabla^{2}\right] E_{T},(\nabla \cdot E=0),
$$

$$
0=\left[\varepsilon(\omega)+\xi^{2}(\omega) \nabla^{2}\right] \boldsymbol{E}_{L},(\nabla \times \boldsymbol{E}=0) .
$$

The latter equation adds wave vector dispersion to the previously discussed bulk plasmon, with $\omega_{L}(\boldsymbol{q})$ being now defined by $0=\varepsilon\left(\omega_{L}\right)+\xi^{2}\left(\omega_{L}\right)(i q)^{2}$. Inserting the Drude dielectric function, we get $\omega_{L}(\boldsymbol{q})=\sqrt{\omega_{p}^{2}+\beta^{2} q^{2}}$. In the same way, we find that $\omega_{T}(\boldsymbol{q})=\sqrt{\omega_{p}^{2}+c^{2} q^{2}}$. For an indepth discussion that classifies and compares both scalar and tensorial nonlocal response models, see Ref. [210].

\subsection{Implications for experiments}

The implications of the hydrodynamic formalism are perhaps best illustrated by considering the polarization of a small metallic sphere of radius $R$. Figure 6 illustrates the induced fields in a sub-wavelength $2 \mathrm{D}$ version of this geometry, with panel (a) indicating how the induced charge density associated with the dipole resonance is smeared over a finite region $(\sim \xi)$ in the near vicinity of the surface (rather than being a strict surface charge as in the LRA), while panel (b) shows the corresponding norm of the electric field. The 3D spherical version of this problem can naturally be treated exactly within Mie theory $[120,189]$. For the dipole resonance, we find to leading-order in $R^{-1}$ that $[120,124,168,183,189]$

$$
\omega_{\mathrm{LSPR}} \simeq \frac{\omega_{p}}{\sqrt{3}}+\frac{\beta}{\sqrt{2} R}=\frac{\omega_{p}}{\sqrt{3}}\left[1+\frac{\xi}{\sqrt{2} R}\right]
$$

which implies that the relative spectral shift is given by

$$
\frac{\Delta \omega_{\mathrm{LSPR}}}{\omega_{\mathrm{LSPR}}} \simeq \frac{\xi}{\sqrt{2} R}
$$

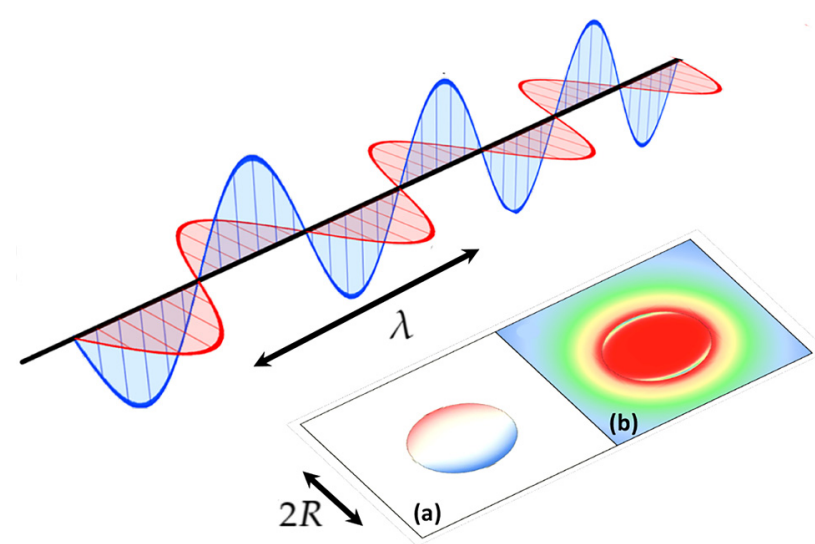

Figure 6: Schematic illustration of excitation of sub-wavelength metallic disk, $R \ll \lambda$, with the $\boldsymbol{E}$-field (in red) polarized in the horizontal plane. (a) Induced charge density $\rho(r)$ of the dipole resonance and (b) corresponding norm of the electric field. 
This is the hydrodynamic nonlocal explanation for the $R^{-1}$ size-dependent shift discussed in the literature for noble metals. While there indeed is a qualitatively good agreement, the experimentally observed blueshifting in Ref. [125] exceeds the predictions of the hydrodynamic model (using generally agreed tabulated Fermi properties of silver [86]). We deliberately refrain from any pragmatic fudge-fitting in the quest of claiming also a quantitative agreement. It is emphasized that the spectral blueshift associated with the finite compressibility of the free electron gas (treated in a hard-wall approximation) is opposite to the redshift characteristic for metals, such as sodium and aluminum, with pronounced spill-out that conflicts with the hard-wall approximation. This apparent problem of opposite spectral shifts derives from the hard-wall approximation, while it is per se not a fundamental issue of hydrodynamics, and self-consistent hydrodynamic models do indeed capture both size-dependent blueshifts and redshifts in silver and sodium particles, respectively [207].

As another consequence, Figure 7 shows how in addition to the blueshifting of the dipole resonance below the plasma frequency, the nonlocal hydrodynamic model also predicts a series of longitudinal resonances above the plasma frequency. These resonances are associated with particle-in-a-box type standing longitudinal plasmon waves confined by the metal surface [189]. Fingerprints of nonlocal hydrodynamic response are constituted by the blueshifting of the dipole resonance and the emergence of bulk-plasmon resonances above the plasma frequency. Naturally, discrete longitudinal resonances can also be confined in other geometries, and Ref. [189] showed that they naturally only appear above the plasma frequency,

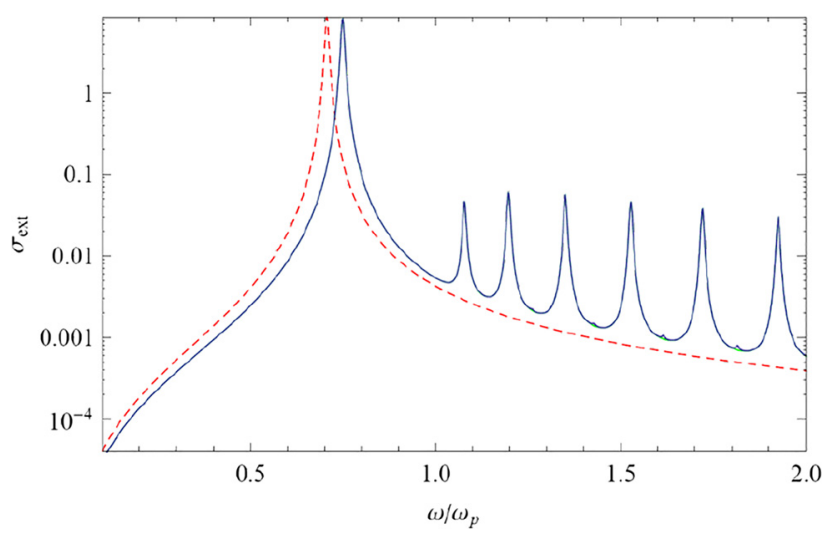

Figure 7: Extinction spectrum of a cylinder of radius $R=2 \mathrm{~nm}$, contrasting the nonlocal hydrodynamic result (solid line) to the local-response approximation (dashed line). The chosen materials parameters resemble gold with $\hbar \omega_{p}=8.812 \mathrm{eV}, \hbar \gamma=0.0752 \mathrm{eV}$, and $v_{\mathrm{F}}=0.0046 \mathrm{c}=1.39 \times 10^{6} \mathrm{~m} / \mathrm{s}$. while being damped below the plasma frequency. Discrete longitudinal resonances in ultra-thin metal films [211] have been experimentally observed in e.g. silver [212] and potassium [213] films.

Turning to dimers of nanoparticles, the tiny gap works essentially as a capacitor, thus hosting an increasing local electrical field as the gap is reduced. In the LRA, there is naturally a diverging and eventually singular behavior $[214,215]$. Here, the nonlocal response redistributes the induced surface charge over a finite region $\xi$ near the surface, thus relaxing the singular response even when the particles are touching [173, 216-219]. This introduces fundamental limitations to the field enhancement, while also posing fundamental limitations for the associated plasmon hybridization [220].

\section{Generalized nonlocal hydrodynamic model - convection-diffusion dynamics}

In this section, we introduce the concept of diffusion into the hydrodynamic description of the nonlocal plasmonic response of the electron gas [168]. The already treated hydrodynamic model is semiclassically accounting for the finite compressibility of the electron gas. Here, we will in addition account for surface-scattering phenomena by introducing an additional relaxation mechanism for the surface-induced charge. While the extension is surprisingly straightforward, the consequences of the additional account for diffusion are far reaching. After having reviewed the formalism, the section will briefly provide such examples.

It should be emphasized that while diffusive transport may perhaps seem like a new innovation in the context of mesoscopic electrodynamics, its importance for the nonlocal response was already anticipated in textbook accounts on spatial dispersion [221]. An important contribution of Ref. [168] was to treat convection and diffusion on an equal footing. The odds are high that this calculation was already worked out in detail by Landau and Liftshitz, while the final result eventually only qualified for an insightful footnote in their Course of Theoretical Physics [221]: "For an isotropic conducting medium, the conditions for spatial dispersion to be negligible may be different for transverse and longitudinal permittivities. For the former, the characteristic distance $\xi$ over which the kernel of the integral in (4) is nonzero is the smaller of $v_{\mathrm{F}} / \omega$ and $\ell$, where $v_{\mathrm{F}}$ is the mean velocity of the carriers and $\ell$ their mean-free path. For the longitudinal permittivity, $\xi$ is the smaller of $v_{\mathrm{F}} / \omega$ and $\left(\ell v_{F} / \omega\right)^{1 / 2}$, the latter 
being the distance traveled by the carriers by diffusion along the field in a time $\sim 1 / \omega$; the diffusion coefficient $\mathscr{D} \sim \ell v_{F}$. The spatial dispersion is unimportant if $k \xi \ll 1$."

Naturally, the convection-diffusion equation is widely exploited in various disciplines within natural and technical sciences (and beyond), including drift-diffusion theory in semiconductor physics [222] as well as fluid mechanics and chemical engineer [77]. In fact, earlier work on nanofluidics and convection-diffusion dynamics in the electrohydrodynamics of electrolytes [223] was indirectly catalyzing the consideration of co-existing transport mechanisms explored in Ref. [168].

\subsection{Convection-diffusion dynamics}

We start from the equation of motion of the original hydrodynamic treatment, Eq. (16), but rather than the usual simple form for the continuity equation, Eq. (18), we now instead invoke the convection-diffusion equation,

$$
\partial_{t} n=\mathscr{D} \boldsymbol{\nabla}^{2} n-\boldsymbol{\nabla} \cdot(n \boldsymbol{v}),
$$

where $\mathscr{D}$ is the classical diffusion constant associated with diffusive transport of the electrons. Again, we invoke the linearization procedure detailed in Eq. (19) and repeat the tedious manipulations that eventually led to Eq. (20). Perhaps not too surprising, with this procedure we arrive at the exact same master equation for the generalized nonlocal optical response (GNOR), only now with a generalized nonlocal length scale [168]

$$
\xi^{2}(\omega)=\frac{\beta^{2}}{\omega(\omega+i \gamma)}+\frac{\mathscr{D}}{i \omega} .
$$

In terms of boundary conditions, the convection and diffusion components of the current entering Fick's law are of the same mathematical form and they are consequently subject to the same boundary conditions [168]. As a result, boundary conditions remain unchanged in the presence of diffusion, giving us $\boldsymbol{n} \cdot \boldsymbol{J}=0$ on the metal surfaces so that no electrons escape the metal volumes.

As already anticipated in Section 2.2, convection and diffusion coexist to form a generalized nonlocal problem with $\xi^{2}=\xi_{\mathrm{conv}}^{2}+\xi_{\mathrm{dif}}^{2}$, where $\xi_{\mathrm{conv}}^{2}=\frac{\beta^{2}}{\omega(\omega+i \gamma)}$ is that of the usual hydrodynamic problem, while $\xi_{\mathrm{dif}}^{2}=\frac{\mathscr{D}}{i \omega}$ is an additional contribution due to diffusion. This is fully along the line of the discussion by Landau and Liftshitz (see quote above), the main contribution of this work being

2 Mathematical expressions have been adapted to the current notation, and the referenced equation is now referring to the corresponding equation in this review. the additional appearance of Euler's imaginary $i$ in the addition of $\xi_{\text {conv }}^{2}$ and $\xi_{\text {dif }}^{2}$. Of course, this is more than a mathematical curiosity, and it will have important physical implications. In passing, we note that the only conceptual change in the formalism is to render $\xi^{2}$ complex valued. As such, all existing work rooted in the usual hydrodynamic description can easily be extended to include diffusion by making the simple substitution $\beta^{2} \rightarrow \beta^{2}-i \mathscr{D}(\omega+i \gamma)$ [168]. This applies not only to analytic treatments e.g. Refs. $[104,120,189]$ but also to numerical implementations, e.g. the finite-element method (FEM) work [173, 194, 224], the discontinuous Galerkin time-domain method [225], the Green's function surface-integral method [226], the Fourier modal method [227], and multipole expansions [228].

\subsection{Physics underlining the diffusion}

Starting from statistical physics and the principle of entropy [221], it is clear that any initial charge inhomogeneity will seek to relax or equilibrate to a state without gradients in the density. Clearly, this can be facilitated by various means, including the mutual interaction of the electrons. Classically, we mimic such complex microscopic phenomena by the diffusion process. Invoking the diffusion equation in the above treatment, we are thus hiding our ignorance about the microscopic details of such manybody interactions behind the Brownian motion of charge carriers by resuming to the introduction of a classical diffusion constant $\mathscr{D}$.

The diffusion constant is generally interlinked with other transport parameters such as the scattering time, e.g. $\mathscr{D} \propto \tau=\gamma^{-1}$. In the context of plasmonics, the diffusion constant $\mathscr{D}$ accounts for the diffusive temporal spreading of an initially pure surface charge into the metal volume of a plasmonic nanoparticle. Diffusion thus degrades plasmonic excitations, providing an additional broadening mechanism that, mathematically, is enacted by an imaginary contribution to $\xi^{2}$. As such, $\mathscr{D}$ captures the effects of both mutual interactions among the electrons and the nonspecular scattering of the electrons on rough metal surfaces. However, it also mimics the surface-enhanced Landau damping due to the creation of electron-hole pairs, as will be discussed in more detail in Section 5 .

The potential misconception that diffusion is slow could motivate its negligence in comparison with convection (see discussion in the quote by Landau and Liftshitz above). Here, we have treated the two mechanisms on an equal footing, allowing the physical relevant parameters to finally decide if one of them would dominate the other, or if they indeed are playing in concert to form a generalized complex-valued nonlocal parameter. 
In which domain is the model valid? For classical gases of charged particles (such as dilute plasma, electrolytes, and weakly doped semiconductors), the velocity distribution is governed by the Maxwell-Boltzmann statistics and the diffusion constant $\mathscr{D}$ is proportional to the temperature $T$, as summarized by the Einstein relation. The primary focus here is, however, metals with a denser electron gas that exhibits other statistics. In this case, Fermi-Dirac statistics implies a narrow transport velocity distribution [221] with a characteristic velocity $v_{\mathrm{F}}$. As a result, the diffusion constant is simply given by $\mathscr{D} \simeq v_{F}^{2} \tau$, corresponding to a mean-free path of $\ell=v_{\mathrm{F}} \tau$. We emphasize that the diffusive model is valid for structural dimensions exceeding the mean-free path. In pure single crystals [229] $\ell$ can be of the order of $100 \mathrm{~nm}$ for silver and gold, down to $\sim 3 \mathrm{~nm}$ for sodium. The reason for mentioning sodium will become clear when later discussing ab initio modeling of plasmons. Moreover, in realistic plasmonic nanostructures, $\ell$ depends on actual material-processing conditions, becoming naturally shorter than in single-crystalline bulk metals. This enlarges the validity domain of a diffusion description to include structures with dimensions of only a few nanometers. For even smaller dimensions with $a<\ell$, electrons will move ballistically between the surfaces of the structure, and surface scattering might become important.

For metals, Eq. (26) simplifies to

$$
\xi^{2}(\omega)=\frac{v_{F}^{2}}{\omega^{2}}\left[\frac{3}{5}-i \frac{\omega}{\gamma}\right]+\mathcal{O}(\gamma / \omega) .
$$

This result enables an important insight into the interplay of different broadening mechanisms: the lower the Ohmic loss and absorption, the more important is the nonlocal response due to long-range diffusion of the induced charge. Re-introducing the diffusion constant $\mathscr{D}$ and the $\beta$-parameter we arrive at the equation

$$
\xi^{2}(\omega) \simeq \frac{\beta^{2}}{\omega^{2}}-i \frac{\mathscr{D}}{\omega}, \quad \ell \gg v_{F} / \omega
$$

which holds if the mean-free path significantly exceeds the convective length. The existence of spatial dispersion in homogeneous media, as for example, appearing within the common hydrodynamic Drude model, can be derived from higher-level descriptions such as the Boltzmann equation, the RPA, or techniques exploited also in plasma physics [230], such as quantum kinetics and hydrodynamics [231]. Beyond hydrodynamics, confined structures with broken translational invariance constitute a largely unexplored territory, with few attempts dealing with RPA aspects of localized plasmon resonances [204]. In support of the prediction of diffusive broadening, both self-consistent hydrodynamic studies [232] and RPA studies have revealed an increased plasmon linewidth associated with Landau damping [233].

Table 1 is summarizing the characteristic length scales for two noble metals, gold and silver, being widely exploited in plasmonics. In addition, the table also includes results for sodium. Though clearly not experimentally attractable, sodium constitutes a good free-electron jellium, which motivates its widespread consideration in $a b$ initio studies within TDDFT [201, 234-238]. The main message is the similar ball game of the convective and diffusive contributions to the nonlocal length scale, being both in the sub-nanometer to nanometer regime. The fact that they are roughly similar drives home the need for the treatment of those two effects on an equal footing.

\subsection{The relation to higher-level descriptions}

Above, we have used the classically widely accepted convection-diffusion model to account for diffusive effects in the hydrodynamic description of plasmons in metallic nanostructures. Here, we briefly mention more recent discussions of this. In particular, it is long known how the hydrodynamic model with the convective contribution can be derived from higher-level descriptions such as the Boltzmann transport equation or Kubo formalism [240, 241]. Can the more recent diffusive contribution be derived in a similar manner? Here, we recall that for convection and diffusion sharing the same underlying microscopic mechanism, their high and low-frequency limits should be intimately connected by the fluctuation-dissipation theorem [78]. Focusing on the convective contribution $\xi_{\text {conv }}^{2}$ as it occurs in the standard hydrodynamic model of a bulk metal, we see that it actually already contains both high-frequency convective and low-frequency diffusive contributions, i.e.

$$
\frac{v_{F}^{2}}{\omega(\omega+i \gamma)} \simeq \begin{cases}\frac{v_{F}^{2}}{\omega^{2}}, & \omega \gg \gamma \\ \frac{v_{F}^{2}}{i \omega \gamma}=\frac{\mathscr{D}}{i \omega}, & \omega \ll \gamma\end{cases}
$$

where $\mathscr{D}=v_{F}^{2} / \gamma$ is the already introduced classical estimate for the diffusion constant. Now, this also indicates that a high-frequency diffusive contribution term would not emerge out of Boltzmann considerations applied to the homogeneous bulk system. While somewhat a lengthy discussion not repeated here, this can indeed also be shown explicitly [242]. Simply stated, bulk diffusion at optical frequencies is negligible. So, what is then the physical mechanism behind the seemingly experimentally observed diffusion at optical frequencies? Here, surfaceenhanced Landau damping represents a surface-scattering effect beyond the bulk description of the metal [154, 156, 
Table 1: Central length scales and parameters for gold ( $\mathrm{Au})$, silver $(\mathrm{Ag})$, and sodium $(\mathrm{Na})$.

\begin{tabular}{|c|c|c|c|c|c|}
\hline & $\begin{array}{r}\text { Fermi wavelength } \\
\qquad \lambda_{\mathrm{F}}\end{array}$ & $\begin{array}{r}\text { Mean-free path } \\
\ell\end{array}$ & $\begin{array}{r}\text { Convection length } \\
\qquad \ell_{\text {conv }}=v_{\mathrm{F}} / \omega_{p}\end{array}$ & $\begin{array}{l}\text { Diffusion length } \\
\ell_{\text {dif }}=\sqrt{\mathscr{D} / \omega_{p}}\end{array}$ & $\begin{array}{r}\text { Scattering time } \\
\omega_{p} / \gamma\end{array}$ \\
\hline \multirow[t]{2}{*}{$\mathrm{Au}$} & $0.52 \mathrm{~nm}$ & $103 \mathrm{~nm}$ & $0.10 \mathrm{~nm}$ & $1.9 \mathrm{~nm}$ & 1000 \\
\hline & $0.52 \mathrm{~nm}$ & $50 \mathrm{~nm}$ & $0.10 \mathrm{~nm}$ & $1.3 \mathrm{~nm}$ & $465^{a}$ \\
\hline \multirow[t]{2}{*}{$\overline{\mathrm{Ag}}$} & $0.52 \mathrm{~nm}$ & $103 \mathrm{~nm}$ & $0.10 \mathrm{~nm}$ & $1.9 \mathrm{~nm}$ & 1000 \\
\hline & $0.52 \mathrm{~nm}$ & $40 \mathrm{~nm}$ & $0.10 \mathrm{~nm}$ & $1.1 \mathrm{~nm}$ & $421^{a}$ \\
\hline \multirow[t]{3}{*}{$\overline{\mathrm{Na}}$} & $0.68 \mathrm{~nm}$ & $2.6 \mathrm{~nm}$ & $0.12 \mathrm{~nm}$ & $0.32 \mathrm{~nm}$ & $21^{a}$ \\
\hline & $0.68 \mathrm{~nm}$ & $4.4 \mathrm{~nm}$ & $0.12 \mathrm{~nm}$ & $0.42 \mathrm{~nm}$ & $37^{b}$ \\
\hline & $0.68 \mathrm{~nm}$ & $2.3 \mathrm{~nm}$ & $0.12 \mathrm{~nm}$ & $0.31 \mathrm{~nm}$ & $20^{c}$ \\
\hline
\end{tabular}

The entries for different metals are based on Fermi wavelengths $\left(\lambda_{\mathrm{F}}\right)$, Fermi velocities $\left(v_{\mathrm{F}}\right)$, and plasma frequencies $\left(\omega_{p}\right)$ taken from Ashcroft and Mermin [86], while the values for $\tau=1 / \gamma$, and consequently also $\mathscr{D} \simeq v_{F}^{2} \tau$, originate from various references as indicated in the

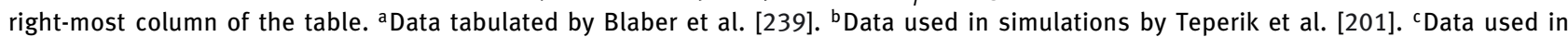
simulations by Stella et al. [237].

157, 243, 244]. In fact, jellium $a b$ initio considerations of sodium-like metal surfaces in Ref. [202] demonstrate this very explicitly.

Finally, we note that an ad hoc inclusion of surface effects in a bulk term should be done with care and one might naïvely speculate that the diffusion constant should then at least be space-dependent to only account for diffusion at the surface [242]. However, since the induced charge density resides only in the near vicinity of the surface without any significant density gradients in the bulk, the diffusion is anyway only effective near the surface. Thus, the pragmatic use of a spatially constant diffusion constant is without practical consequences.

\subsection{Implications for experiments}

\subsubsection{Unified description of size-dependent frequency blueshifts and line broadening}

As a first example of the physical implications, we reiterate that the two transport mechanisms together form a complex-valued $\xi^{2}$, with the real part being predominantly attributed to the convection, while the imaginary part originates from the diffusion. For the dipolar LSPR of a metallic sphere of radius $R$, one immediately finds $R^{-1}$ size-corrections to $\omega_{\mathrm{LSPR}}=\omega_{\mathrm{LSPR}}^{\prime}+i \omega_{\mathrm{LSPR}}^{\prime \prime}$ given by [120, $124,168,183,189]$

$$
\begin{gathered}
\omega_{\mathrm{LSPR}}^{\prime} \simeq \frac{\omega_{p}}{\sqrt{3}}+\frac{\beta}{\sqrt{2} R}, \\
\omega_{\mathrm{LSPR}}^{\prime \prime} \simeq-\frac{\gamma}{2}-\frac{\sqrt{6} \mathscr{D} \omega_{p}}{24 \beta R} .
\end{gathered}
$$

These expressions for the dipolar resonance transpire clearly that the $R^{-1}$ size-dependent nonlocal effects are present in both the resonance frequency and linewidth. It should be emphasized that, until the publication of Ref. [168], line shifts have been explained by the nonlocal response (and competing theories), whereas the line broadening was 'put in by hand'. Here, we have arrived at a unified explanation of both experimentally observed phenomena in noble metal nanoparticles by nonlocal effects. Line broadening has been seen experimentally in the extinction of small particles [145, 148, 245-250]. In particular, this is the generalized nonlocal optical response (GNOR) explanation for the $R^{-1}$ size-dependent shift and broadening discussed in the experiments on silver nanoparticles, such as Ref. [145]. We note that for a given size, the size-dependent blueshift is in the same order of magnitude as the size-dependent broadening, thus justifying the need for treating both effects on an equal footing. This is what the GNOR seeks to do by accounting for both convection and diffusion [168]. In fact, the Kreibig model $\omega_{\text {LSPR }}^{\prime \prime} \simeq-\frac{\gamma}{2}-\frac{A v_{F}}{R}$, where experimentally $A \sim 1$, can be immediately used to estimate $\mathscr{D}$. Indeed, this procedure gives values of $\mathscr{D}$ that are in rough accordance with the independent data in Table 1.

In passing, EELS explorations of silver nanoparticles and their higher multipoles have likewise exhibited sizedependent damping [124], with an angular-momentum dependence [120] not anticipated by the phenomenological $A v_{\mathrm{F}} / R$ term of the Kreibig model [148]. EELS measurements on plasmons in thin nanowires and bow-tie antennas have also revealed plasmon losses exceeding the expectations based on bulk-damping parameters [98, 251]. Helped by the PDE formulation, the GNOR can naturally also be used to numerical account for size-dependent damping in nanostructures of more complex surface morphology beyond mere spherical symmetry. 
As already emphasized in Section 3.6 for the hydrodynamic model, also the GNOR model invokes the assumptions of the hard-wall approximation, thus foremostly reaching out to the experimentally relevant cases where noble metals with relatively high work functions are being used. Without enforcing any self-consistency (such as in Ref. [207]), the GNOR is on the other hand inadequate for metals with pronounced spill-out effects, such as the case of sodium that is being widely considered as a model system in ab initio accounts [82, 164, 201, 236, 252, 253].

\subsubsection{Hybridization and gap-dependent broadening in nanoparticle dimers}

The previous subsection has established contact between experimental observations on monomers and the GNOR model, showing how a finite $\mathscr{D}$-value can mimic sizedependent damping. Next, the crucial check is to explore whether the GNOR model would also serve to explain spectra of dimers. In particular, can experimentally observed gap-dependent broadening [159, 162, 254] in dimers be explained using the very same choice of $\mathscr{D}$-value?

Figure 8 shows an example where the gap $g$ is reduced to the nonlocal regime $g \lesssim v_{\mathrm{F}} / \omega$. Here, resonances are slightly blueshifted with respect to the LRA result. When the gap shrinks further, progressively stronger hybridization [135] and accordingly larger bonding-dipole plasmon (BDP) redshifts are clearly seen. At the same time, the BDP is gradually suppressed owing to the increasing role of diffusion as the contact point is approached $(g=0)$. This is in strong contrast to predictions from both the LRA [214] (with even diverging field enhancement) and from previous nonlocal theories that treated the $\beta$-parameter real-valued [173].

As one enters the contact regime, the BDP fades away, vanishing completely for $g<0$. We emphasize that the negative gap values $(g<0)$ correspond to overlapping wires, in which case conducting nanojunctions are formed. In this way, the charge can flow back and forth in a classical manner rather than being transferred in a quantum tunneling fashion. We emphasize that, for $g \sim 0$, the diffusive broadening is so strong that only higher-order modes persist (as the induced surface charge is located away from the contact point), while both the BDP and the CTP are strongly suppressed. This makes a discussion on their possible coexistence problematic [160]. Finally, we note that in the anticipated tunneling regime [82] the extinction spectra are strongly broadened by the complex nonlocal response. In fact, the semiclassical approach is in remarkable agreement with the TDDFT results [201, 236, 237], with

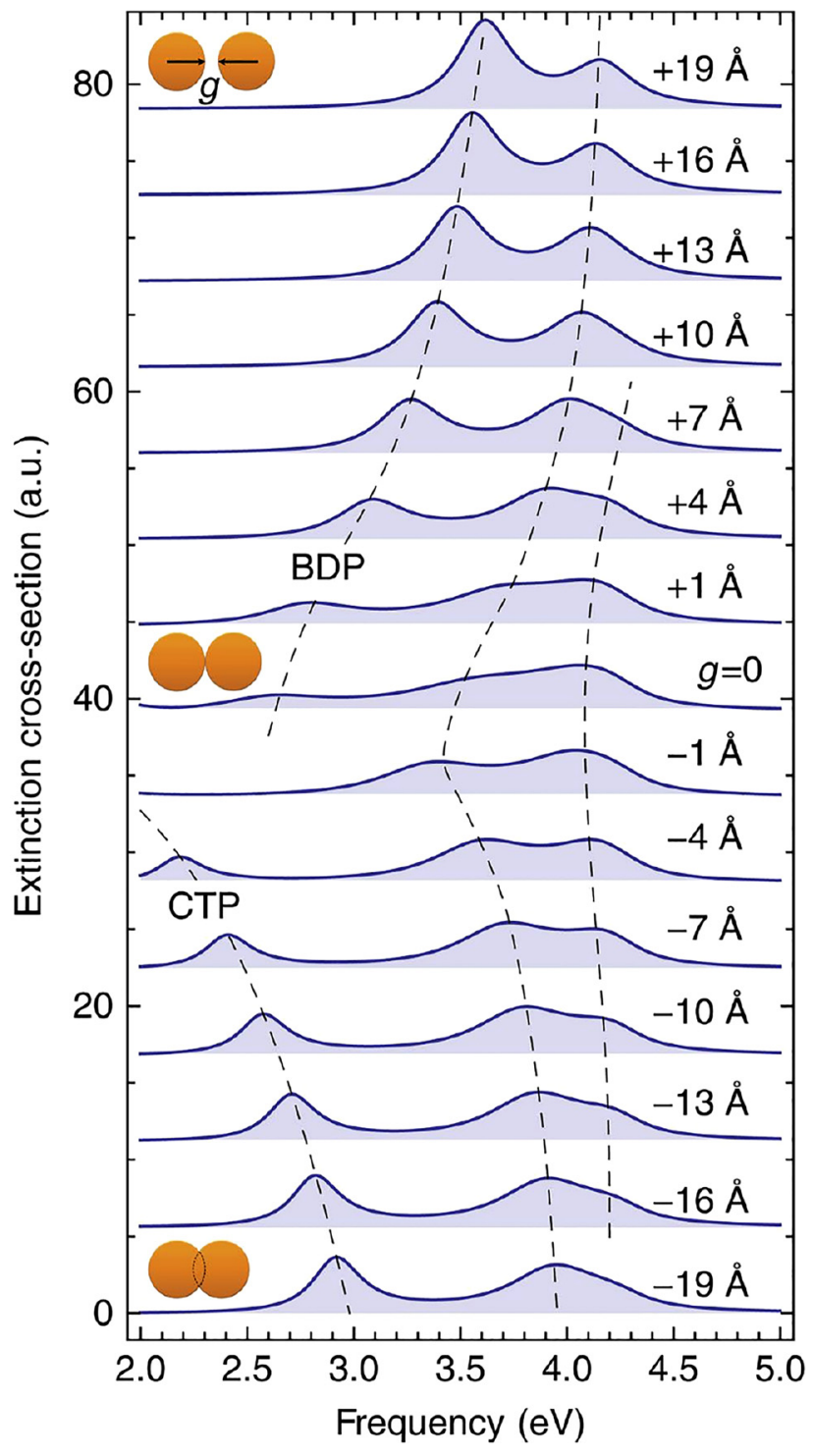

Figure 8: Hybridization and gap-dependent broadening in sodium nanowire dimers. The radius of the sodium wires is $R=4.9 \mathrm{~nm}$ with the gap $g$ varying from -19 to $+19 \AA$. Progressively stronger hybridization occurs as the gap narrows, with both a clear redshift and broadening of the bonding-dipole plasmon. As the gap closes, the charge transfer plasmon (CTP) develops and blueshifts as the wires start to overlap $(g<0)$. Higher-order modes (also indicated by dashed lines) exhibit hybridization and broadening too. The diffusion $\left(\mathscr{D}=1.36 \times 10^{-4} \mathrm{~m}^{2} / \mathrm{s}\right)$ causes GNOR spectra in accordance with time-dependent density-functional theory calculations of Ref. [201] and in overall agreement with the experimental observation of the CTP $[159,160,254-256]$ and its broadening. Figure reproduced from Ref. [168] (C) 2014 Nature Publishing Group, a division of Macmillan Publishers Limited).

the diffusion contribution being responsible for 'repairing' the apparent incompatibility of TDDFT calculations and earlier hydrodynamic predictions [201, 236]. 
In the above context, it is therefore important to stress that both the LRA and GNOR spectra are obtained with an entirely semiclassical model where the simplifications and the resulting boundary conditions (see Section 3.3) do not consider a quantum-tunneling interpretation. Most critically, the work function is considered infinite and the metal surfaces serve as hard walls or perfectly reflecting mirrors for the electrons so that inter-nanoparticle charge transport is entirely prohibited for $g>0$. Instead, the gap-dependent broadening is due to surface scattering as mimicked by the diffusion contribution to the nonlocal response. In this way, size-dependent broadening becomes pronounced as the gap approaches the magnitude of the nonlocal length scale. Note that broadening also occurs for weakly overlapping wires; a case where quantum tunneling is suppressed, with the charge-transport being of a classical nature. To summarize, gap-dependent broadening sets in for nanometric dimer gaps and GNOR spectra agree qualitatively with both experiments [159, 254] and ab initio studies [201].

So, what is the important common feature of the monomers and the dimers? Clearly, tunneling has no meaning for monomers. On the other hand, if surface scattering appears near any metal surface, then this effect could explain both the enhanced scattering at the surfaces of monomers as well as the scattering near the surfaces of almost touching dimers. This being said, although quantum tunneling is systematically excluded from the semiclassical theory, one cannot rule out the possibility that quantum tunneling may lead to additional gap-sizedependent broadening in dimers, as an additional physical mechanism of spectral broadening [202]. In Section 5, we turn to $a b$ initio studies [202] where more rigorous insight consolidates the importance of surface-enhanced Landau damping (mimicked as diffusive broadening). In particular, we show how this indeed acts as a unifying feature of both monomers and dimers.

\section{Surface-response formalism}

The previous sections have focused on nonlocal corrections to the LRA that enter the electrodynamics at the PDE level through an additional differential operator term. In this section, we curiously explore the possibilities for instead including the corrections through quantumcorrected boundary conditions, while maintaining the LRA form of the PDE itself. This has stimulated a renewed interest in electrodynamic surface-response functions $[80,257-261]$.
In the spirit of the LRA, the previous sections have considered the situation where metal domains are characterized by their bulk properties which extend all the way to the metal surface. As an example, the equilibrium density of free electrons $n_{0}(\boldsymbol{r})$ was considered homogeneous and given by $n_{0}$ inside the metal, while dropping abruptly to zero at the surface. When this problem is then in turn perturbed by a time-varying $\boldsymbol{E}$-field, the induced charge density $\varsigma$ resides in the near vicinity of the surface, being a strict surface-charge density in the LRA, while in the nonlocal hydrodynamic description it is being smeared over a finite region of extension $\xi$. In more general terms, we may divide a given metal domain into its interior bulk part (implicitly assuming that the domain is large enough to exhibit a bulk part), being well accounted for by bulk optical parameters, while surrounded by an assumed thinner transition region between the bulk part on the one side and the exterior vacuum on the other side. Sipe refers to this transition region at the surface as the selvage [262], which includes the classically defined surface of the metal domain.

In the zoom-in of Figure 9, we encounter an example of the selvage in the context of the jellium description of the equilibrium electron density, where the surface region is hosting both Friedel oscillations and the evanescent tail associated with quantum spill-out. The rationale behind the results of Refs. [122, 202] is to treat the bulk part of the electrodynamics classically, while dressing this problem with quantum-corrected boundary conditions that account for the nonlocal phenomena hosted in the selvage. This was also the motivation for the earlier work by Luo et al. [263]. The inherent assumption is naturally that the selvage is of a small extension relative to all other problems involved in the classical electrodynamic problem, including of course the light wavelength $\lambda$ and the characteristic dimensions $R$ of the bulk part of the problem. As such, we are indeed looking for surface corrections to the classical bulk problem. It should be emphasized that this idea is well-rooted in also the experimental understanding of the problem, with deviations from the classical electrodynamics being associated with surface-scattering phenomena [220, 264, 265]. Naturally, the selvage is present at any metal surface, while its significance is promoted in nanoscale structures with large surface-to-volume ratios.

\subsection{The concept of Feibelman parameters}

In order to quantify the dynamics in the selvage illustrated in Figure 9, we will now introduce the framework of Feibelman [80], where $\varrho(\boldsymbol{r}, \omega)$ denotes the induced charge 


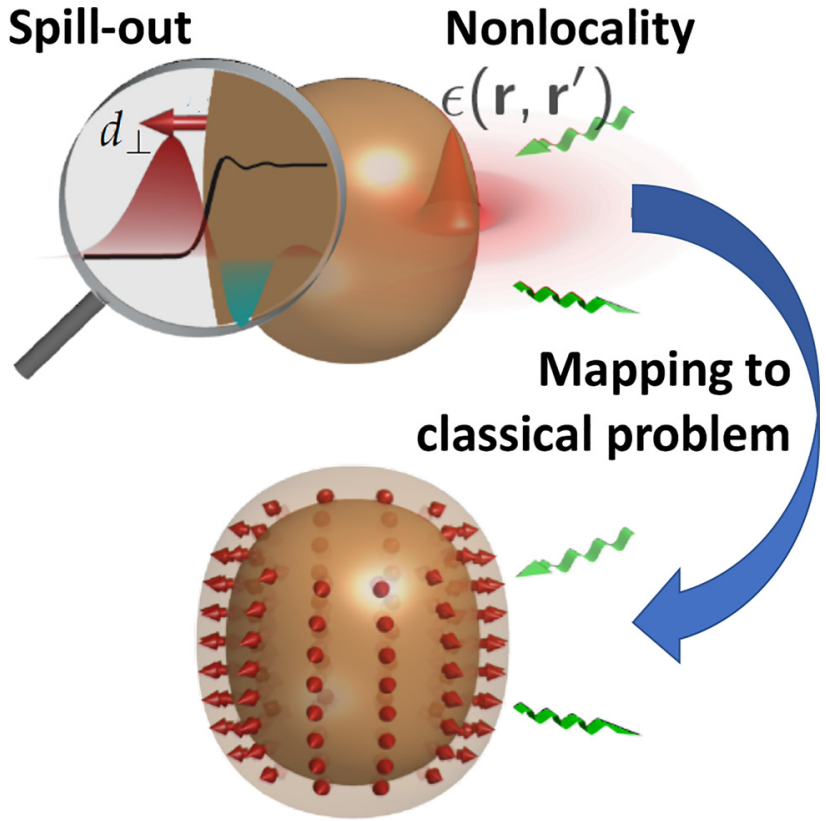

Figure 9: Light-driven quantum-plasmonic response in a metallic nanostructure (upper panel) is represented in classical electrodynamics by an infinitely thin layer of dipoles that point perpendicularly to the surface (lower panel). The metallic domain has an interior bulk part and a selvage region at the surface, constituting a transition region between the bulk and the exterior vacuum. The magnifying glass (upper panel) zooms in on the selvage, highlighting both the equilibrium electron density (black solid line), and also the microscopic distribution of the induced charge $\rho$ (red/green filled curve) which is inducing a dipole moment (red arrow) proportional to the Feibelman parameter $d_{\perp}$.

density that occupies the selvage region. In the context of the previous hydrodynamic descriptions, we would have $\varrho(\boldsymbol{r}, \omega)=n_{1}(\boldsymbol{r}, \omega)$, while we for historical reasons here proceed with the former notation. In the equilibrium case, the Friedel oscillations in $n_{0}(\boldsymbol{r})$ occur on the Fermi wavelength scale in the vicinity of the surface, and as we have already seen in the hydrodynamic treatment, the dynamic induced surface charge $n_{1}(r, \omega)$ is smeared on a length scale of $\xi$, being sub-nanometric for the metals. Thus, the extension of the selvage is indeed much smaller than any other length scales involved in the classical electrodynamic problem.

The net-induced surface-charge density $\varsigma$ is then given by $\varsigma(\omega)=\int \mathrm{d} x \rho(x, \omega)$, where the coordinate system is now chosen so that $x$ is in the direction of the surface normal, while later $y$ will be in a direction parallel to the surface. While the LRA treats the induced surface charge as a strict surface charge, i.e. $\rho(x, \omega)=\varsigma(\omega) \delta(x)$, the idea is to now represent a more general spatial distribution of the charge by its first moment $d_{\perp}(\omega)$, which accounts for the centroid of the induced charge. In this way, the leadingorder quantum effect is to induce a dynamic dipole moment proportional to $d_{\perp}(\omega)$ in the surface region. The association with a dipole moment naturally provides an important hint that this can be used as input for quantum-corrected mesoscopic boundary conditions to be exploited in subsequent classical treatment of the electrodynamics. This representation of quantum corrections is referred to as the projected-dipole model (PDM) [202], being schematically illustrated in the right-hand panel of Figure 9. While this first Feibelman parameter, $d_{\perp}(\omega)$, is sufficient to describe most problems invoking metal surfaces, we for completeness also note the existence of an additional parameter $d_{\|}(\omega)$, which accounts for the possible surface conduction by surface states [80]. In this way, the surface response is now parameterized by the so-called Feibelman $d$-parameters [80]

$$
\begin{gathered}
d_{\perp}(\omega)=\frac{\int \mathrm{d} x x \varrho(x, \omega)}{\int \mathrm{d} x \varrho(x, \omega)}, \\
d_{\|}(\omega)=\frac{\int \mathrm{d} x x \partial_{x} J_{y}(x, \omega)}{\int \mathrm{d} x \partial_{x} J_{y}(x, \omega)} .
\end{gathered}
$$

We emphasize that the dispersive surface-response functions will generally be complex-valued, $d_{\perp}=d_{\perp}^{\prime}+i d_{\perp}^{\prime \prime}$ and $d_{\|}=d_{\|}^{\prime}+i d_{\|}^{\prime \prime}$, with real and imaginary parts being related through Kramers-Kronig relations. While $d_{\|}(\omega)=0$ in the absence of surface states, it could potentially be finite for surfaces supporting Shockley surface states, e.g. (111) surfaces of noble metals, or when surfaces are being covered by conducting molecular layers, e.g. 2D graphene-like materials or other molecular films with in-plane order. We shall return to both examples in the later discussion in Section 7. In passing, we note that of course, the induced surface charge and surface current would depend on the amplitude of the electrical field, but in the spirit of linearresponse theory, the Feibelman parameters are naturally independent of this assumed small amplitude. We note that alternatively to Eq. (31), the $d$-parameters can also be written in terms of surface integrals associate with the difference between the actual microscopic fields and the classical Fresnel fields stemming from the PCA in Eq. (6) [80, 260, 266-268].

Finally, we note that in the above consideration we have implicitly assumed that the surface topography varies slowly on the scale of the perpendicular extension of the selvage, thus effectively neglecting curvature effects. In Section 5.4.1, we will further explore this assumption. 


\subsection{Mesoscopic boundary conditions for classical electrodynamics}

We now turn to the problem of formulating quantumcorrected boundary conditions that would allow the account for Feibelman parameters in classical electrodynamics. The key observation is that $d_{\perp}(\omega)$ represents a dipole moment. Just like any surface charge would enter the regular electromagnetic boundary conditions [14], the presence of a dipole layer and surface currents would do so too.

The somewhat lengthy derivation and outcome of Ref. [202] is the mesoscopic boundary conditions in Eq. (32), which were recently generalized to include both $d_{\perp}(\omega)$ and $d_{\|}(\omega)[269]$

$$
\begin{gathered}
\llbracket D_{\perp} \rrbracket=d_{\|} \boldsymbol{\nabla}_{\|} \cdot \llbracket \boldsymbol{D}_{\|} \rrbracket, \\
\llbracket B_{\perp} \rrbracket=0, \\
\llbracket \boldsymbol{E}_{\|} \rrbracket=-d_{\perp} \boldsymbol{\nabla}_{\|} \llbracket E_{\perp} \rrbracket, \\
\llbracket \boldsymbol{H}_{\|} \rrbracket=i \omega d_{\|} \llbracket \boldsymbol{D}_{\|} \rrbracket \times \boldsymbol{n} .
\end{gathered}
$$

Here, we have adapted the notation of Ref. [269] where $\llbracket \boldsymbol{F} \rrbracket \equiv \boldsymbol{F}^{+}-\boldsymbol{F}^{-}$denotes the discontinuity of any vector field $\boldsymbol{F}$ across an interface $\partial \Omega$ with outward normal vector $\boldsymbol{n}$.

The clear benefit of this approach is that the mesoscopic boundary conditions can be applied in two and three dimensions to any system size that is already tractable within classical electrodynamics and with the use of standard computational photonics tools [166] while capturing quantum plasmonic aspects of nonlocal response and a finite work function with TDDFT-level accuracy. It should be emphasized that the challenge of the alternative full TDDFT treatment [253] relates to the computational complexity and the practical applicability to mainly small plasmonic systems [201, 234, 235, 238, 270].

\subsection{How to determine Feibelman parameters?}

Having reviewed the framework that allows the incorporation of quantum-corrected boundary conditions in classical treatments of the electrodynamics, we are next facing the challenge of determining the Feibelman parameters for relevant metal surfaces. There might be an impression that being a microscopic concept, surface-response formalism calls for $a b$ initio time-dependent treatments of the surface in order to extract dynamic surface-response functions. However, any relaxation of either the LRA (Eq. (9)) or the PCA (Eq. (6)) will lead to a situation with a finite surface response. To appreciate this, we note that for a spatially varying local dielectric function $\varepsilon(x)$, the induced charge $\rho(x)$ is given by [271]

$$
\frac{1}{\varepsilon_{0}} \varrho(x) \propto \frac{\partial \varepsilon^{-1}(x)}{\partial x},
$$

thus illustrating how an induced charge density occupies surface regions where the dielectric function changes.

\subsubsection{Local-response approximation with equilibrium electron-density profile}

For simplicity, we entirely focus on the intraband response of the electron gas itself. Using RPA arguments, it can be shown that the Drude bulk response of a homogeneous electron gas (Eq. (8b)) generalizes to [80, 204, 272-275]

$$
\varepsilon(x, \omega)=\overbrace{\Theta(-x)}^{\begin{array}{c}
\text { Metal } \\
\text { background }
\end{array}}+\overbrace{[\varepsilon(\omega)-1] \Pi(x)}^{\text {Electron jellium }}+\overbrace{\Theta(x)}^{\begin{array}{c}
\text { Surrounding } \\
\text { vacuum }
\end{array}},
$$

where the first term represents the background of the jellium (for simplicity neglecting interband transitions). In the second term, $\Pi(x) \equiv n_{0}(x) / n_{0}$ is a normalized equilibrium density function approaching unity deep in the bulk of the metal, while vanishing in the vacuum away from the metal surface. Physically, this can be interpreted as the common Drude response, but with a spatially varying plasma frequency. The third term represents the surrounding vacuum. We emphasize that this concept of a spatially varying plasma frequency has found broad use, including Refs. [203, 205, 271, 276-281]. The idea of a smoothly varying profile has also been considerations of local-field corrections [282].

To illustrate the effects of spill-out, we consider the smooth profile of the equilibrium electron density $\Pi(x)=$ $\tanh ^{2}\left(\frac{x-x_{0}}{a}\right) \Theta\left(x_{0}-x\right)[271,283]$, where $a$ is the characteristic length over which the electron density drops. The Heaviside function terminates the electron density beyond $x=x_{0}$, where the sign of $x_{0}$ captures whether the induced electron density spills inward or outward. Physically, this model mimics a jellium metal surface (such as sodium) with a moderate work function, which allows the quantum spill-out of the equilibrium electron density beyond the surface of the metal. The model qualitatively agrees with results obtained by jellium considerations within density-functional theory (DFT) [187], while $n_{0}(x)$ is here for simplicity smooth, thus neglecting Friedel oscillations $[187,188,199,284]$. Only for $x_{0}=a$ the symmetry of the model makes the surface charge neutral. 
In passing, we note how interpolation formulas of the jellium-surface problem [285] can be used to conveniently link $a$ to the Wigner-Seitz radius $r_{s}$ of the electron gas. For densities relevant to noble metals $\left(r_{s} \sim 4 a_{0}\right)$ we have $a \sim 0.15 \times \lambda_{\mathrm{F}}$ [285]. Following the procedure in Ref. [271], this particular equilibrium density profile gives

$$
\begin{gathered}
d_{\perp}(\omega)=x_{0}-a \frac{\arctan (\sqrt{\varepsilon(\omega)-1})}{\sqrt{\varepsilon(\omega)-1}}, \\
d_{\|}(\omega)=x_{0}-a .
\end{gathered}
$$

Here, the parametrization in terms of the Drude dielectric function $\varepsilon(\omega)$ underlines how the surface-response functions inherit their frequency dispersion from that of the bulk counterpart. Figure 10A illustrates the generic dependence associated with the spill-out, where $d_{\perp}^{\prime}(\omega)>d_{\|}^{\prime}(\omega)$ at low frequencies, while $d_{\perp}^{\prime}(\omega)<d_{\|}^{\prime}(\omega)$ at higher frequencies as one approaches the plasma frequency. It should be emphasized that the above result is solely the surface contribution from the relaxation of the PCA to a spatially varying equilibrium electron density, while the electrodynamics is still being treated within the LRA.

\subsubsection{Nonlocal semiclassical hydrodynamic model}

Next, we consider the surface contribution from a relaxation of the LRA to a nonlocal hydrodynamic description, while still maintaining the PCA. Having already promoted the semiclassical hydrodynamic model in Section 3, where the induced charge is smeared over a length scale $\xi$, one could speculate that $d_{\perp}(\omega)$ should somehow resemble $\xi(\omega)$. Indeed the following expressions [242]

$$
\begin{gathered}
d_{\perp}(\omega)=-\frac{\xi(\omega)}{\sqrt{-\varepsilon(\omega)}}, \\
d_{\|}(\omega)=0,
\end{gathered}
$$

describe the surface response of the hydrodynamic jellium surrounded by vacuum $[122,202]$, with $d_{\perp}$ representing the centroid of the induced charge $\rho$, e.g. visualized in panel (a) of Figure 6 for the dipole resonance treated within the hydrodynamic model. As illustrated in Figure 10B, this also emphasizes the point that $d_{\perp}(\omega)$ will be complexvalued just as $\xi(\omega)$ was found complex-valued within the GNOR model that we considered in Section 4. Perhaps somewhat ironically, the numerically more complicated hydrodynamic models (where the numerical mesh needs to resolve dynamics on the $\xi$ length scale in the vicinity of the surface [173]) can now be solved within the numerically much more tractable LRA problem, while instead of
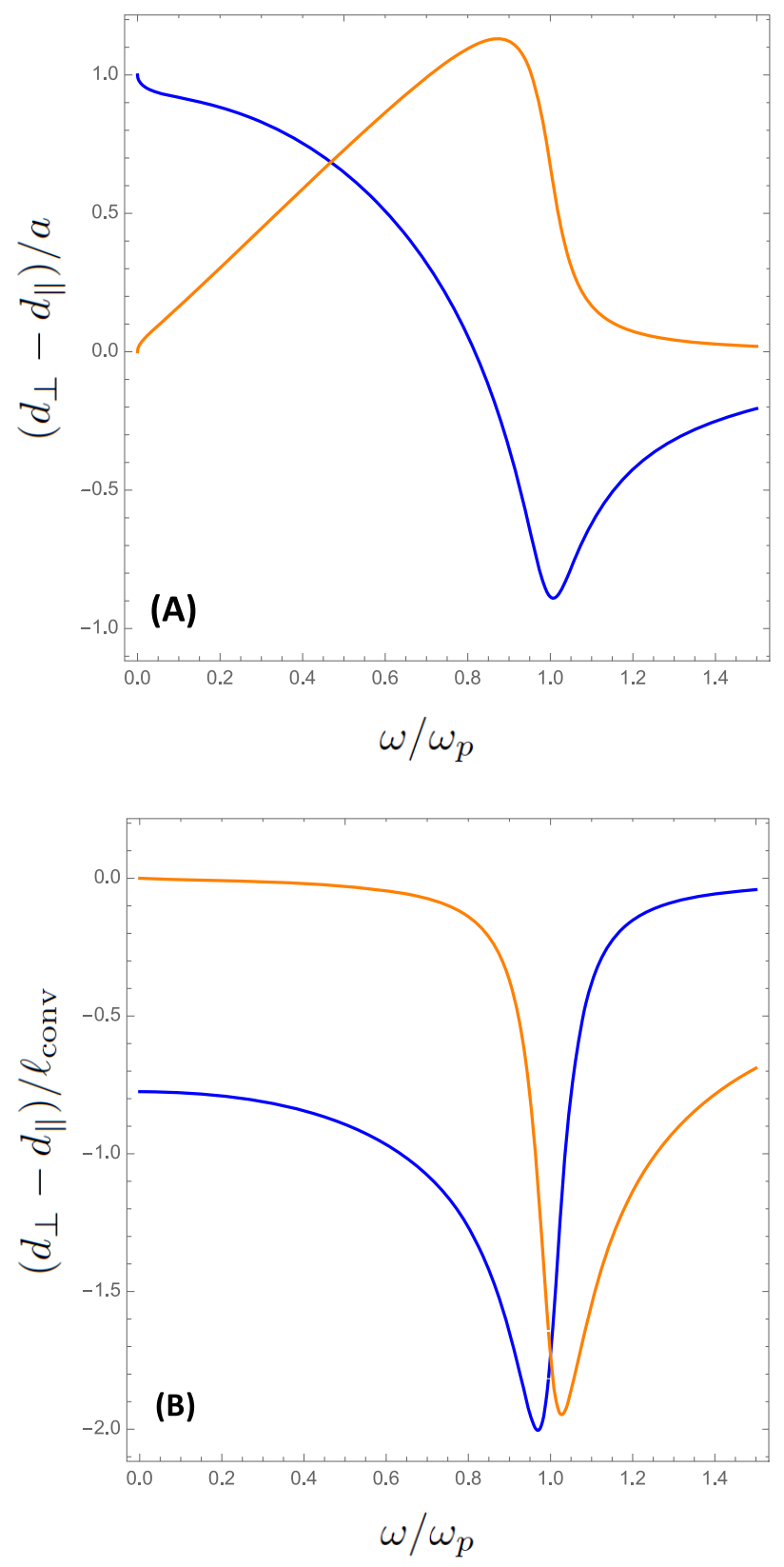

Figure 10: Spectral dependence of the frequency-dispersive effective Feibelman parameter $d_{\perp}-d_{\|}$for $\gamma / \omega_{p}=0.1$, where the real part (in blue) and the imaginary part (in orange) are related through Kramers-Kronig relations. (A) Local-response approximation contribution from a smooth equilibrium electron-density profile, Eq. (35) normalized by $a$. (B) Nonlocal hydrodynamic contribution, Eq. (36) normalized by $\ell_{\text {conv }}=v_{\mathrm{F}} / \omega_{p}$.

applying the mesoscopic boundary conditions, Eq. (32). In passing, we note that different hydrodynamic boundary conditions at the metal surface would naturally be reflected in different surface-response functions too [286]. 


\subsubsection{First-principles jellium model}

Next, let us proceed one level deeper in the description of the dynamic response of the quantum electron gas, by turning to the jellium model that we already discussed in the context of the equilibrium properties of the semi-infinite jellium. In the treatment of the dynamical properties, we turn to the TDDFT, basically evaluating the response of the semi-infinite jellium in the presence of a time-harmonic $\boldsymbol{E}$-field [202]. This has allowed numerical evaluation of $d_{\perp}(\omega)=d_{\perp}^{\prime}(\omega)+i d_{\perp}^{\prime \prime}(\omega)$ for specific values of the Wigner-Seitz radius $r_{s}$ [202]. Note that in this specific case, the charge-neutral surface makes $d_{\|}$vanish.

Figure 11 shows results for electron densities resembling e.g. aluminum and sodium. The general trend is that $d_{\perp}^{\prime \prime}(\omega)$ increases with frequency, peaking slightly beyond the plasma frequency as a manifestation of surfaceenhanced Landau damping. Being microscopic linearresponse functions, the Kramers-Kronig relation naturally causes a corresponding frequency dispersion for $d_{\perp}^{\prime}(\omega)$ [41], with a positive value signifying the quantum spill-out of the induced charge. For the more realistic modeling of e.g. silver, $d$-band screening should be taken into account $[122,199]$. The accurate evaluation of the Feibelman parameters seems to remain a general challenge within the TDDFT community.

\subsubsection{Beyond jellium descriptions}

Clearly, it would also be interesting to proceed beyond jellium models by invoking atomistic descriptions [287]. Here, the challenge of atomistic details has been tackled in $a b$ initio studies of the response of metallic clusters and gaps [288, 289]. As another example, atomic-layer pseudo-potentials [290] have recently been invoked in RPA descriptions of plasmons in noble-metal films [291] and the same approach has been used to also evaluate Feibelman parameters [292]. In the future, one would ultimately even benefit from experimentally determined values [269], tabulated for different metal surfaces in the same way that the bulk parameters have been tabulated. Section 7.5.1 will briefly discuss the prospects of this, using added graphene layers [293] as an experimental probe of nonlocal effects in the metal [294, 295].

\subsection{Physical implications}

The Feibelman parameter $d_{\perp}(\omega)=d_{\perp}^{\prime}(\omega)+i d_{\perp}^{\prime \prime}(\omega)$ immediately parameterizes two important quantum effects, i.e. the electron spill-out via $d_{\perp}(\omega)$ and the surface-enhanced
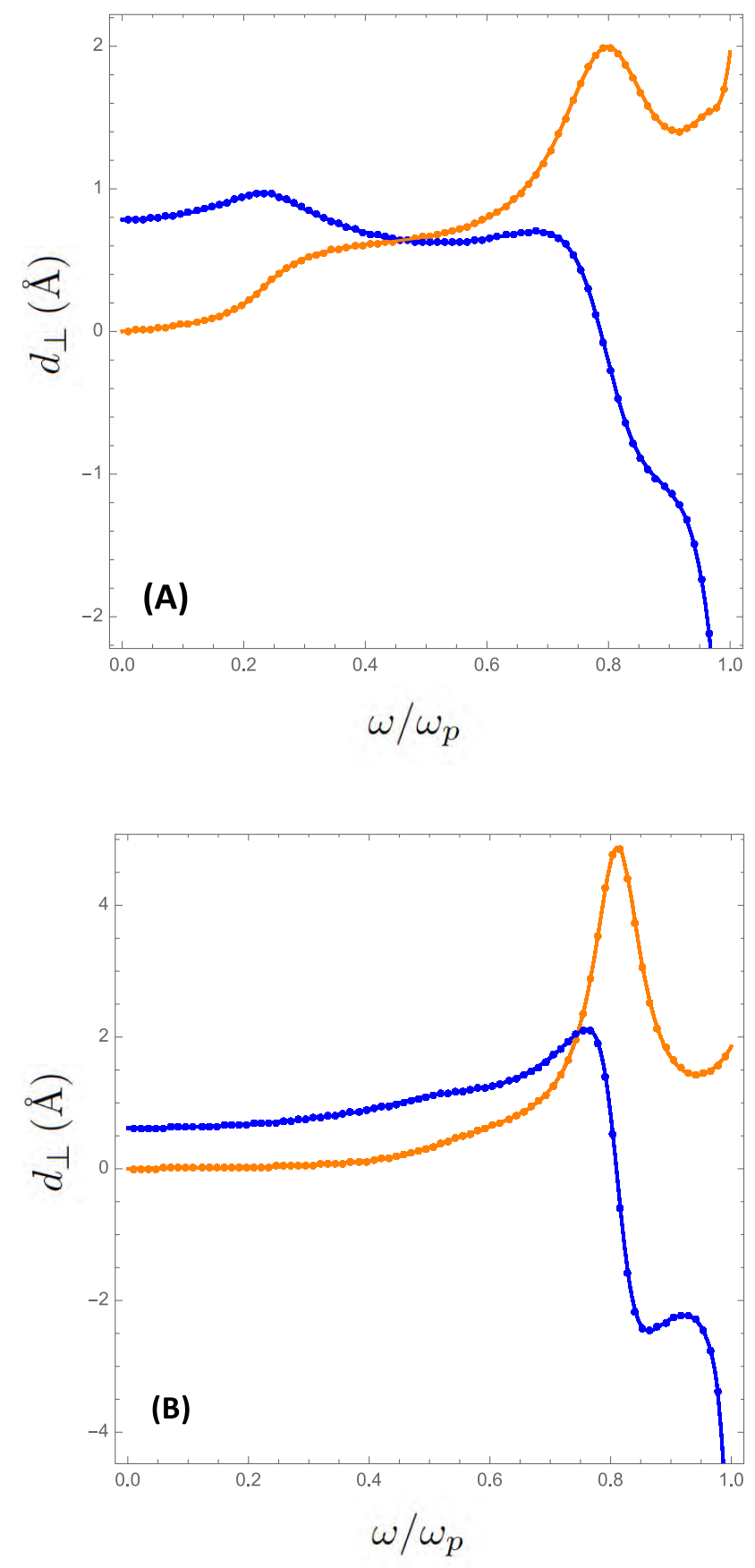

Figure 11: Spectral dependence of the frequency-dispersive effective Feibelman parameter $d_{\perp}$ for jellium metals with different Wigner-Seitz radius, where the real part (in blue) and the imaginary part (in orange) are related through Kramers-Kronig relations. (A) Jellium with $r_{s} / a_{0}=2$ typical for noble metals. (B) Jellium with $r_{s} / a_{0}=4$ typical for sodium. Figure based on data from Ref. [296].

Landau damping associated with electron-hole $(\mathrm{e}-\mathrm{h})$ excitations via $d_{\perp}^{\prime \prime}(\omega)$. However, since the $a b$ initio TDDFT treatment inherently accounts for also the finite compressibility of the electron gas and its influence on transverse 
modes, the extracted Feibelman parameters include also effects of the nonlocal dynamics of the bulk, that the semiclassical hydrodynamic models account for. Of course, the hydrodynamic models also include longitudinal modes (see e.g. Figure 7 above the plasma frequency), that the Feibelman parameters are leaving out by construction. The link from the hydrodynamic bulk description to the surface-response approach is highlighted by Eq. (36), while the opposite attempt at deriving hydrodynamic parameters from surface-response functions is more ambiguous [242].

With Eq. (36) in mind, it is of course immediately clear that the present formalism can qualitatively explain both the size-dependent frequency shifts and broadening in monomers, as well as the gap-dependent broadening in dimers. In the following sections, we show that it even works qualitatively when benchmarking to full quantum treatments within the TDDFT of the jellium model. Subsequently, we use the established correspondence with $a b$ initio TDDFT calculations, to explore quantum plasmonic response for structural dimensions that are beyond any reach of $a b$ initio methods. While we are here focusing on the implications manifested through computational studies, we will in Section 6 turn to a path where several analytical results for the leading-order plasmonic quantum corrections are obtained in a first-principles setting.

\subsubsection{Benchmarking against full TDDFT results}

Having extracted the Feibelman parameters from the semiinfinite jellium model, Figure 11, it is next interesting to turn the attention to a finite-size nanostructure formed from the very same jellium. In fact, this will allow for a unique headto-head comparison between the current PDM approach (treating the bulk classically, while invoking the quantumcorrected boundary conditions) and a full TDDFT solution (treating the entire volume quantum mechanically).

Figure 12 shows extinction spectra for sodium nanowires, contrasting the results from LRA, PDM, and TDDFT. For the latter, we are relying on TDDFT results reported independently by Teperik et al. [201] for jellium parameters resembling sodium. In panel (a), one first observation is naturally the significant change in spectra due to the quantum corrections, which serve to redshift the dipole resonance [297], when compared to the LRA spectral position. Perhaps more important in the present context, we see an amazing quantitative agreement between the PDM and the full TDDFT. The main approximation is here the negligence of curvature effects, but even for this somewhat extreme example with $R \sim 5 \mathrm{~nm}$, this is obviously not a severe approximation.

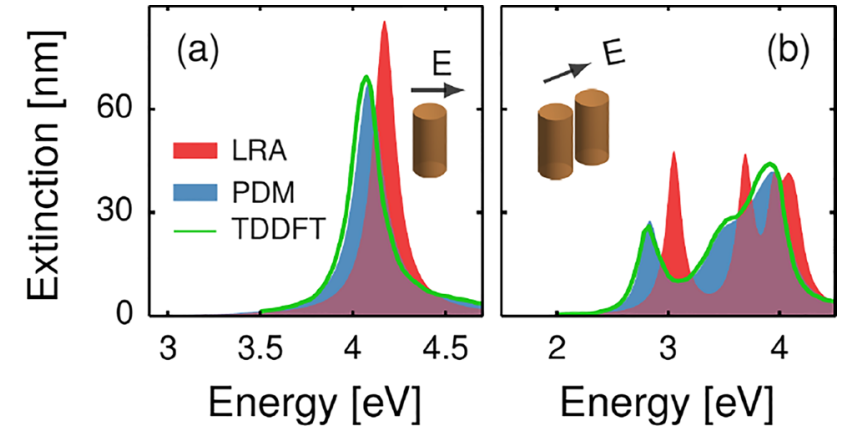

Figure 12: Extinction spectra for sodium monomers and dimers, contrasting results from local-response approximation (red-filled curve), projected-dipole model (blue-filled curve), and time-dependent density-functional theory (TDDFT) (green solid line). (a) Cylindrical wire with radius $R=4.9 \mathrm{~nm}$, (b) cylindrical wires with radius $R=4.9 \mathrm{~nm}$ and gap $g=0.74 \mathrm{~nm}$. The choice of these specific geometrical parameters is motivated by the corresponding availability of the TDDFT results extracted from Ref. [236]. Figure reproduced from Ref. [202] (C) 2015 American Physical Society).

Turning next to the dimer made from the very same wires, now with a $g=1 \mathrm{~nm}$ gap, panel (b) shows similar quantitative agreement between the PDM and the full TDDFT, thus emphasizing the extreme potential of the PDM for computational quantum plasmonics.

In the context of the dimers, it should be noticed that naturally the PDM prohibits the account for any possible charge transfer between the wires, while the tunneling mechanism is naturally fully contained in the full TDDFT treatment. Nevertheless, the potential existence of opticalfrequency tunneling currents does not seem to be important in the present case of a $1 \mathrm{~nm}$ gap. Given the tremendous attention to quantum tunneling in nanogap dimers $[82,159,164,254,298]$, this is a perhaps somewhat surprising observation that we will return to in Section 7.

\subsubsection{Quantum effects promoted by nanogaps in large nanosphere dimers}

Having established that the PDM excellently reproduces the results of the numerically much more demanding full TDDFT treatment, let us next turn to problems that are simply too extensive for any TDDFT treatment of the dynamics [253]. Here, Figure 13 shows results for sodium nanosphere dimers where the sphere radius extends even beyond $R=25 \mathrm{~nm}$. As expected, the radius is far too large to promote any significant $R^{-1}$-corrections. Instead, the subnanometric gap is promoting significant gap-dependent corrections, causing very significant quantum corrections to the LRA spectrum. This can be well appreciated in a 


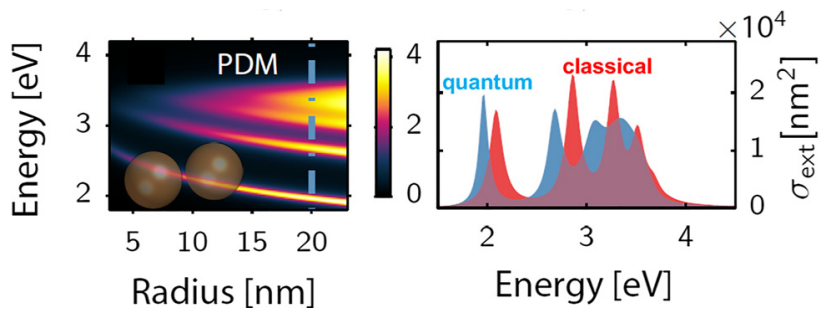

Figure 13: Extinction spectra for sodium nanosphere dimers, contrasting results from local-response approximation (LRA) (red-filled curve) and the projected-dipole model (blue-filled curve). Despite the relatively large radius $R$ of the spheres, being far too large to promote significant size corrections to the LRA, the tiny gap $g=0.74 \mathrm{~nm}$ is in itself sufficient to promote significant gap-dependent corrections. Figure reproduced from Ref. [202] (C) 2015 American Physical Society).

simple hybridization picture [135], with a change in the effective gap separating the now slightly smeared charge densities induced on the opposing metal surfaces, i.e. $g \rightarrow g-2 d_{\perp}^{\prime}=g\left(1-\frac{2 d_{\perp}}{g}\right)$.

\section{Surface-integral approach to quantum corrections}

While Section 5 focused on the introduction of microscopic surface-response functions and the formulation of mesoscopic boundary conditions that enable computational explorations, this section summarizes the efforts of Ref. [122] in establishing the analytical means for deeper insight into general properties of the quasi-static regime. It almost goes without saying that the concept of surfaceresponse functions goes hand-in-hand with Green's function surface-integral methods [299], which have already been explored in the context of the hydrodynamic model $[226,300,301]$. Here, we aim for the leading-order quantum correction terms to the classical surface-integral eigenvalue equation for metallic domains in the quasi-static regime [302, 303].

\subsection{Surface-integral eigenvalue equation}

Considering the quasi-static regime, where $\lambda \gg R$ and retardation effects can be neglected, the spectral response of the plasmons can be explored through a surface-integral eigenvalue equation for the induced surface charge $\rho$; this is the LRA part of Eq. (37) below. As a main outcome of Ref. [122], this LRA surface-integral eigenvalue equation is complemented by leading-order corrections due to the Feibelman surface-response functions

$$
\begin{aligned}
& \text { Surface-integral eigenvalue equation (LRA) } \\
& \overbrace{\Lambda \varsigma(\boldsymbol{r})=\mathscr{P} \int_{\partial \Omega} d^{2} \boldsymbol{r}^{\prime}\left[\boldsymbol{n} \cdot \nabla g\left(\boldsymbol{r}, \boldsymbol{r}^{\prime}\right)\right] \varsigma\left(\boldsymbol{r}^{\prime}\right)} \\
& +\underbrace{d_{\perp} \lim _{\delta \rightarrow 0^{+}} \int_{\partial \Omega} d^{2} \boldsymbol{r}^{\prime}\left[\boldsymbol{n} \cdot \boldsymbol{\nabla} \boldsymbol{\nabla}^{\prime} g\left(\boldsymbol{r}+\delta \boldsymbol{n}, \boldsymbol{r}^{\prime}\right) \cdot \boldsymbol{n}^{\prime}\right] \varsigma\left(\boldsymbol{r}^{\prime}\right)}_{\text {Quantum spill-out \& surface-enhanced Landau damping }} \\
& -\underbrace{d_{\|} \int_{\partial \Omega} d^{2} \boldsymbol{r}^{\prime} \boldsymbol{\nabla}_{\|}^{2} g\left(\boldsymbol{r}, \boldsymbol{r}^{\prime}\right) \varsigma\left(\boldsymbol{r}^{\prime}\right)}_{\text {Conductive surface states }}
\end{aligned}
$$

Here, $g\left(\boldsymbol{r}, \boldsymbol{r}^{\prime}\right)=\left|\boldsymbol{r}-\boldsymbol{r}^{\prime}\right|^{-1}$ is a scalar Green's function that represents the finite-range Coulomb interactions. In the LRA part, $\mathscr{P}$ denotes the Cauchy principle-value integration, while in the $d_{\perp}$-correction term, $\boldsymbol{\nabla} \boldsymbol{\nabla}^{\prime}$ is the Hessian with elements $\left\{\nabla \nabla^{\prime}\right\}_{\alpha \eta}=\partial_{\alpha} \partial_{\eta}$ and $\alpha=\{x, y, z\}$ and $\eta=\left\{x^{\prime}, y^{\prime}, z^{\prime}\right\}$.

Most importantly, Eq. (37) constitutes an eigenvalue problem with $\Lambda$ being a scale-independent dimensionless eigenvalue (or a set of such eigenvalues) that is unique to the morphology of a given geometry. Once determined, the eigenvalue $\Lambda$ in turn provides the resonance frequency through the definition

$$
\Lambda=\frac{\varepsilon_{d}(\omega)+\varepsilon(\omega)}{\varepsilon_{d}(\omega)-\varepsilon(\omega)} .
$$

which somewhat resembles an inverse Clausius-Mossotti factor, see Eq. (11a).

Equation (37) forms an interesting starting point for implications of the quantum corrections to the spectral properties of plasmonic nanostructures, with a particular focus on the interplays of morphology, scale, and materials [122]. As we shall see, the LRA part of Eq. (37) is clearly containing the essential information about morphology and in turn the bulk-materials properties, via Eq. (38). In addition, the correction terms associated with the Feibelman parameters reflect the scale and the surface-to-volume ratio, while the surface-response functions naturally also represent materials' properties.

\subsection{Physical implications}

\subsubsection{Universality of the LRA}

Substituting the Drude LRA result in Eq. (8) into Eq. (38), we may straightforwardly solve for the complex frequency 
$\omega=\omega^{\prime}+\mathrm{i} \omega^{\prime \prime}$. Assuming $\gamma \ll \omega_{p}$, this gives the universal LRA relation (for simplicity $\varepsilon_{d}=1$ )

$$
\begin{gathered}
\omega^{\prime} \simeq \sqrt{\frac{1}{2}(1+\Lambda)} \omega_{p}, \\
\omega^{\prime \prime}=-\frac{\gamma}{2} .
\end{gathered}
$$

between the resonance frequency $\omega^{\prime}$ and the morphology eigenvalue $\Lambda$. Likewise, Eq. (39b) illustrates the wellknown result of the linewidth reflecting the bulk-damping parameter $\gamma$, while being independent of the morphology [121]. Beyond quasi-statics [304], a related bound on plasmon damping also exists [305].

Different morphologies lead to different eigenvalues and resonance frequencies, but they all fall on a common square root curve associated with Eq. (39a). Moreover, we notice how this result is entirely independent of the scale of the structures, i.e. the LRA exhibits no size dependence in the quasi-static regime, as we have already discussed in the context of spherical particles.

As an example, the spherical geometry has eigenvalues $\Lambda=-(2 l+1)^{-1}[122]$, where $l=1,2,3, \ldots$ is the angular momentum of the multipoles. For the lowest-energy dipolar resonance $(l=1)$, the corresponding eigenvalue $\Lambda$ $=-1 / 3$ immediately gives the well-known result $\omega=$ $\omega_{p} / \sqrt{3}$ for the dipolar spherical nanoparticle resonance that we already found in the context of the Fröhlich condition in Eq. (11b). The sphere represents the most compact simply-connected domain (with lowest surface-to-volume ratio) and as such the spherical geometry represents also a lower bound for monomers, while any more complex morphology that follows from a surface deformation of the sphere would be associated with a larger eigenvalue and a higher resonance frequency.

Structures with hybridization of plasmon modes can of course reach eigenvalues below the bound of the spherical geometry. As an example, the flat open surface (semiinfinite half-space) is associated with an eigenvalue of $\Lambda=0$ [122], corresponding to the well-known SPR at $\omega=\omega_{p} / \sqrt{2}$ that also followed from Eq. (10b). When such two opposing surfaces hybridize to form an MIM structure, the otherwise degenerate eigenvalues split symmetrically and shift away from $\Lambda=0$, eventually reaching $\Lambda= \pm 1$ in the extreme zero-gap case, corresponding to bonding and antibonding resonances at $\omega=0$ and $\omega=\omega_{p}$. Naturally, the nonlocal effects will prohibit this extreme limit [104] and there will be a fundamental limit to the hybridization as also seen for dimer configurations [220]. Similar physics also manifest when the MIM structure is formed by a metallic nanoparticle on a metallic substrate
- the nanoparticle-on-a-mirror geometry - with additional in-plane localization of electromagnetic fields [109].

\subsubsection{Nonclassical geometry-dependent corrections}

Turning now to the leading-order correction terms in Eq. (37), we immediately see how surface-response functions serve to break the universality of the LRA discussed above. The approach of Ref. [122] is to treat the correction terms to the LRA perturbatively. For the shift in eigenvalues,

$$
\Delta \Lambda=\varkappa_{\perp} d_{\perp}+\varkappa_{\|} d_{\|}+\mathcal{O}\left(d_{\perp}^{2}\right)+\mathcal{O}\left(d_{\|}^{2}\right)
$$

this introduces geometry-dependent perturbation factors $\varkappa_{\perp}$ and $\varkappa_{\|}$, which have dimensions of inverse length, thus representing effective wave numbers. ${ }^{3}$ In passing, we note that negative and positive definiteness, $\varkappa_{\perp}<0$ and $\varkappa_{\|}>0$, can also be proved [122].

In the following, we will mainly focus the discussions on situations with $d_{\|}=0$, while we will in Section 7 discuss extensions to situations with surface conduction mediated by e.g. Shockley surface states or surface-added 2D materials and long-range ordered molecular films.

Turning next to $\omega(\Lambda)$, the shift in eigenvalue $\Delta \Lambda$ will cause a shift in frequency $\Delta \omega \simeq \frac{\partial \omega}{\partial \Lambda} \Delta \Lambda$, and from Eq. (38) we arrive at [122]

$$
\frac{\Delta \omega}{\omega} \simeq \frac{1-\varepsilon(\omega)}{4} \varkappa_{\perp} d_{\perp}
$$

where we have used that for the Drude case $\frac{4}{\omega} \frac{\partial \omega}{\partial \Lambda}=1$ $-\varepsilon(\omega)$. Since $\varkappa \propto R^{-1}$, with $R$ now being some characteristic size of the metallic domain, this result immediately shows an $R^{-1}$-dependence of the frequency shift in the presence of a finite $d_{\perp}=d_{\perp}^{\prime}+i d_{\perp}^{\prime \prime}$, being a redshift for $d_{\perp}^{\prime}>0$ or a blueshift for $d_{\perp}^{\prime}<0$. Furthermore, we also see similar $R^{-1}$-dependent broadening due to $d_{\perp}^{\prime \prime}$.

For a sphere of radius $R$, it can be shown that $\varkappa_{\perp}$ $=\frac{2 l(l+1)}{(2 l+1) R}[122]$, thus showing the $R^{-1}$-dependence explicitly. Cylinder, slab, and gap geometries can also be solved analytically, showing in those translation-invariant cases that $x$ is indeed proportional to the wave vector of the light field [122]. More arbitrarily shaped geometries, possessing no obvious symmetries, will generally prohibit analytical progress, while the LRA part of Eq. (37) can be discretized by the boundary-element method [303] allowing the numerical calculation of the nonclassical shape factors $\varkappa_{\perp}$ and $\varkappa_{\|}$. As an example, Figure 14 shows numerically evaluated morphology and shape factors for a number

3 We have here used $x$ to explicitly emphasize the wave vector nature, while Ref. [122] used the notation $\Lambda^{(1)}$. 
(a)
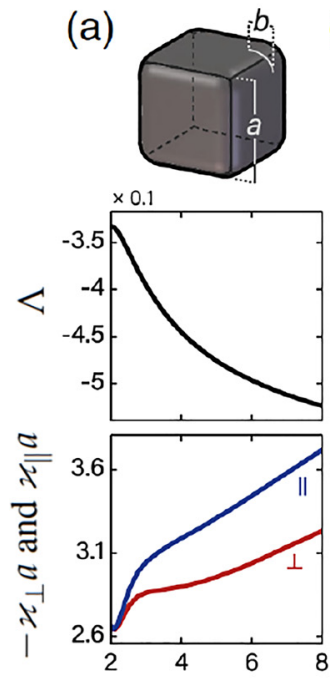

(b)
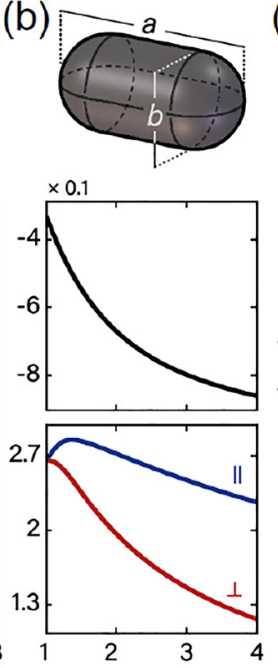

(c)
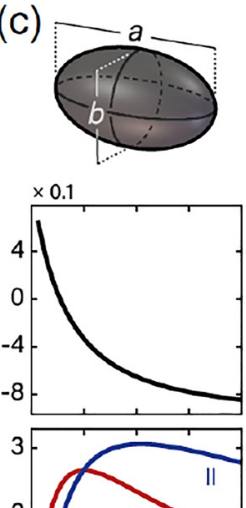

(d)
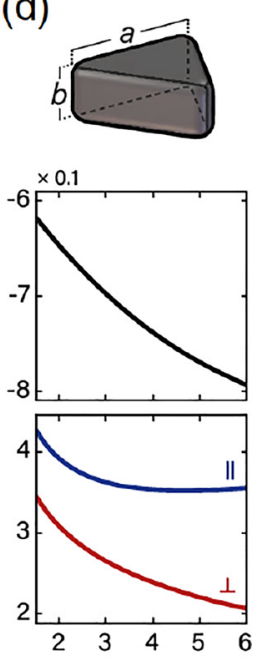

Figure 14: Numerically evaluated morphology and shape factors, $\Lambda, \varkappa_{\perp} a$, and $\varkappa_{\|} a$, for varying aspect ratio $a / b$. Note how the size-dependent shape factors have been normalized by the characteristic length scale $a$.

(a) Cubes of side $a$ and edge- and cornerrounding $2 b$, (b) cylindrical pills of length $a$, diameter $b$, and butt-rounding $b$, (c) spheroids with principal axis $a$ and $b$, and (d) equilateral triangles of height $b$, side $a$, and edge- and corner-rounding $\approx 0.165 a$. A dipole mode is considered in all cases; its induced dipole is along $a$ in (a)-(c) and along the triangle altitude in (d). Rounding is intramural and of a cylindrical and spherical kind with inscribed diameters equaling the specified rounding value. Figure reproduced from Ref. [122] (C) 2017 American Physical Society). of simply connected geometries with varying aspect ratios. For compact geometries with an aspect ratio $a / b \sim 1$, we note how $\Lambda$ attains negative values in the vicinity of the eigenvalue for the sphere, i.e. $\Lambda=-1 / 3$.

Finally, the combination of detailed surface-response functions, as exemplified by the Feibelman parameters in Figure 11, with the morphology and shape factors, as exemplified in Figure 14, naturally forms the basis for evaluating quantum corrections in arbitrarily shaped geometries. The conclusion is that in addition to the simple morphology dependence (described by $\Lambda$ ), the rich interplay between scale, shape, and material in quantum nanoplasmonics can be understood quantitatively through just three parameters: the characteristic scale $R$, the Feibelman $d$ parameter, and the $\varkappa$ shape-factor. As a key result, Eq. (41) illustrates the spectral shifts that directly links to the experimental observations of size-dependent frequency shifts and broadening.

We will conclude this discussion by returning to the already discussed Clausius-Mossotti expression for the dipolar resonance $(l=1)$, Eq. (11a), which in the presence of surface response generalizes to $[296,306]$

$$
\begin{aligned}
\alpha(\omega)= & 4 \pi R^{3} \\
& \times \frac{\left[\varepsilon(\omega)-\varepsilon_{d}(\omega)\right]\left[1+\frac{d_{\perp}(\omega)+2 d_{\|}(\omega)}{R}\right]}{\varepsilon(\omega)+2 \varepsilon_{d}(\omega)-2\left[\varepsilon(\omega)-\varepsilon_{d}(\omega)\right] \frac{d_{\perp}(\omega)+2 d_{\|}(\omega)}{R}} .
\end{aligned}
$$

Here, the associated Fröhlich condition now depends on the effective Feibelman parameter $d_{\perp}-d_{\|}$, that we have already discussed in Figure 10, and in combination with $R^{-1}$, thus illustrating the promotion of $R^{-1}$ size-dependent frequency shifts and damping. Considering the reflection from planar surfaces, the effective Feibelman parameter $d_{\perp}-d_{\|}$again appears in generalized Fresnel coefficients $[80,296]$.

\section{Discussion and outlook}

The above colloquial-style presentations of semiclassical hydrodynamic theory and microscopic surface-response formalism have largely focused on developments in the recent decade, while obviously not doing justice to the many individuals and collective contributions that have all served to advance the science in this research area. This section seeks to partly compensate for this by offering brief perspectives on these developments. Here, it should immediately be emphasized how competing for efforts in abinitio TDDFT treatments of the quantum plasmonic response in metallic nanostructures, in particular by the groups of Nordlander, Aizpurua, and Borisov (see Ref. [82] and references therein), have provided a tremendous push and inspiration to the developments of simpler semiclassical models and the more recent revival of surface-response formalism.

Finally, this discussion and outlook section also turns to a number of topics and directions that are pertinent to the discussion of quantum and nonlocal responses in metallic nanostructures. The section naturally also serves as an outlook to possible future work, with new avenues where nonlocal plasmonic response and surface-response functions will in one or the other way be important to the explorations and harvesting of electrodynamics. Naturally, 
some aspects might turn out to be more of a speculative nature.

\subsection{Perspectives on semiclassical theory developments}

Obviously, it is today difficult for anyone to claim strong fame to basic developments in nonlocal hydrodynamics of electron systems, which was founded almost one century ago by Bloch [49] and important contributions have since then appeared continuously, going also beyond bulk phenomena [196, 307-310]. In particular, Ruppin contributed a seminal paper on the Mie treatment of spherical particles [307] but was perhaps a little ahead of experimental developments in nanoplasmonics. With the later advent of metallic nanostructures, the hydrodynamic approach has in the recent decade had a revival. As it is often the case, García de Abajo was timely and among the first-movers in this new wave [311]. However, the attempts of numerically resolving nonlocal effects in arbitrarily shaped geometries through real-space formulations were sparked by McMahnon et al. [312]. Ref. [189] was published as a direct response to the work by McMahnon et al., providing rigorously formulated real-space formulations with physically transparent boundary conditions. This clarified intriguing predictions by McMahnon et al. [312-315] as nonphysical artifacts of approximations. Admitted, our preliminary conference contribution was guilty in that crime too [316]. Above this initial scientific dispute, Ref. [189] also provided the foundation of numerous later works.

Perhaps most importantly, Eq. (20) formed the basis for the first numerical implementation using the FEM [173] and a first self-consistent hydrodynamic treatment of also quantum spill-out [207]. For recent comparisons of hydrodynamic models, see Ref. [317, 318]. Another novel branch was pioneered by Pendry and co-workers with a marriage of transformation optics and nonlocal plasmonics [217, 319], which aided significant analytical progress and understanding into singular geometries.

While this review begins with an introduction of the generalized nonlocal wave equation, the chronological order of events was different. In fact, the first idea to transform the IDE into an ordinary PDE followed significant attention to the basic hydrodynamic formulation [189] and was only in turn catalyzed by the phenomenological treatment of the two-point response function by Ginzburg and Zayats [100]. Irrespective of the character and origin (microscopical, semiclassical, or phenomenological) of the two-point response function, the IDE remains numerically unattractive. In addition to the conceptually appealing aspect of bringing the nonlocal IDE problem onto a PDE form very close in spirit to the LRA, Eq. (13) has also implications for numerical solutions, since the problem has mathematically now become of a seemingly local nature. This enabled the FEM numerical evaluation of nonlocal effects in extreme plasmonic waveguiding [194, 320]. Most importantly, the generic form of the nonlocal correction term in the PDE was an immediate source of inspiration for the subsequent developments of the GNOR formalism [168].

Prior to the GNOR model [168], convective-diffusive transport was independently studied by Davis and Krowne [321] and later Hanson [322]. Those earlier studies were perhaps more of mathematical curiosity and with less emphasis on the physics of metal nanostructures and their nonlocal plasmonic response. Continuing the path initiated by Landau and Liftshitz [221], the GNOR approach treated convection and diffusion on an equal footing and with immediate implications for the ability to semiclassically account for the nonlocal response associated with both the quantum compressibility of the electron gas and surface-enhanced Landau damping in arbitrarily shaped geometries, thus providing a foundation of numerous later works, including also numerical implementations of the model [225, 228, 323]. In turn, Ref. [168] also enabled a semiclassical explanation (in terms of surface-enhanced Landau damping mimicked by diffusive scattering) of the broadening of the BDP in nanogap dimers, which other works attributed to quantum tunneling [82, 159, 164, 254]. Later, $a b$ initio work in Ref. [202] gave the jelliumbased quantitative insight that consolidated the potential importance of surface-enhanced Landau damping in gap structures.

Finally, in recent years there has been significant activity by Ciracìand co-workers in quantum hydrodynamics invoking energy functionals of increasing complexity [324, 325], thus going beyond the initial self-consistent treatments relying on Thomas-Fermi theory with von Weizsäcker gradient corrections [207].

\subsection{Revival of surface-response formalism}

As it should hopefully be clear from the above introduction to surface-response formalism, credit should go to the surface-science community for early studies of the equilibrium properties of jellium surfaces by Lang and Kohn [187], later motivating also explorations of temporal dynamics and plasmons in jellium with spherical-symmetry confinement [326, 327]. In particular, Feibelman should be recognized for introducing the surface-response functions 
in the first place [328], while there were many contemporaries contributing to this development (see Ref. [329] and references therein).

Naturally, $d$-parameters have been explored in the context of spherical particles, where analytical progress is feasible, while there was for many years modest attention from the computational physics community. With the formulation of mesoscopic boundary conditions, Ref. [202] has changed this. The PDM is combining the computational efficiency of classical electrodynamics with the accuracy of $a b$ initio TDDFT while allowing us to address quantum corrections in geometries far too large for $a b$ initio treatments. As an example of this, the mesoscopic boundary conditions have now been implemented in commercially available FEM codes, allowing for the quantitative evaluation of quantum corrections in complex 3D plasmonic nanoresonators, and with a detailed comparison to experiments [269]. The idea of Ref. [202] was partly inspired by work in the group of Pendry [263], where a finite-thickness local layer represents the hydrodynamic nonlocal response (see Ref. [330] for a generalization), while the PDM goes beyond and capture quantum phenomena of a microscopic theory of choice.

Finally, the TDDFT calculations pursued in Ref. [202] have also caused a paradigm shift in the long-standing discussions on the possible importance of quantum tunneling in nanogap dimers. This will be further addressed in Section 7.4.

The LRA considerations of plasmon resonances by Wang and Shen [121] are seminal, showing several universal properties within the QSA, such as the resonance linewidth being independent of the nanostructure form of the dielectric environment. Ref. [122] is rooted in this quasistatic regime, exploiting first-principles surface-response functions to analytically derive leading-order plasmonic quantum corrections to the LRA electrodynamics of subwavelength plasmonic resonators.

Being the first work to systematically elaborate on a clear separation of the roles of shape, scale, and material, Ref. [122] has naturally inspired further analytical work for both localized and propagating plasmons, including recent studies of quantum corrections in the context of plasmon-emitter interactions [32] as well as the quantum effects arising in the hybridization of $2 \mathrm{D}$ materials with metal surfaces and guidance of plasmons in ultrathin metal films [295]. Refs. [122, 202] have largely focused on the manifestation of surface-response functions with an emphasis on the extinction, in turn stimulating also near-field means of probing surface-response functions, including quantum emitters [296] and 2D materials [295] placed in the near vicinity of the metal surface. Finally, Refs. [122, 202] also paved the way for collaborators' breakthrough in a first experimental attempt at directly measuring the nonclassical surface-response functions [269]. Hopefully, this is a precursor for a future broad tabulation of experimentally extracted surface-response functions of practically relevant materials that can be exploited in quantitative mesoscopic electrodynamic computations.

\subsection{Landau damping}

The publication of Ref. [168], with the introduction of a classical diffusion term, stimulated an interest in the microscopic origin of this diffusive contribution. In simple terms, the diffusion constant $\mathscr{D}$ is the pedestrian way of classically representing the Brownian motion of induced charges that occur due to the probably much more complex mutual interactions of the electrons in the near vicinity of the surface. As such, we are hiding our ignorance of these complex dynamics by sweeping all the microscopic details under the carpet of classical diffusion theory. The diffusive contribution to $\xi^{2}$ being imaginary implies a close connection to Landau damping and the decay of plasmons into electron-hole pairs. Quoting Khurgin from the discussions at the 2015 Faraday Discussions on nanoplasmonics [331]: "Kreibig scattering and Landau damping are essentially two faces of the same process."

Landau damping occurs for wave vectors exceeding $\omega / v_{\mathrm{F}}$ and while perhaps not much of an issue in the bulk of the metal (with a local translational invariance), the abrupt termination of the electron gas near the surface enhances the Landau damping. This can be appreciated from the Fourier transform of even the equilibrium electron-density profile (see Figure 15), representing Fourier components exceeding $\omega / v_{\mathrm{F}}$.

To illustrate this more explicitly, we turn to $a b$ initio solutions of the jellium surface [202]. To assist the illustrations, we introduce the concept of a local effective permittivity $\varepsilon_{\text {eff }}(\boldsymbol{r}, \omega)$ defined by

$$
\boldsymbol{D}(\boldsymbol{r}, \omega) \equiv \varepsilon_{\mathrm{eff}}(\boldsymbol{r}, \omega) \boldsymbol{E}(\boldsymbol{r}) .
$$

The $\varepsilon_{\text {eff }}(\boldsymbol{r}, \omega)=\varepsilon_{\text {eff }}^{\prime}(\boldsymbol{r}, \omega)+i \varepsilon_{\text {eff }}^{\prime \prime}(\boldsymbol{r}, \omega)$ so defined is called "effective" because both the displacement and the electric fields are determined using a fully nonlocal-response theory, and it is the effectively local quantity $\varepsilon_{\text {eff }}(\boldsymbol{r}, \omega)$ that by definition connects the two electromagnetic fields, i.e. close in spirit to the LRA of Eq. (2a). We emphasize that there are no approximations involved in this; it is merely a mathematical convenient choice, 

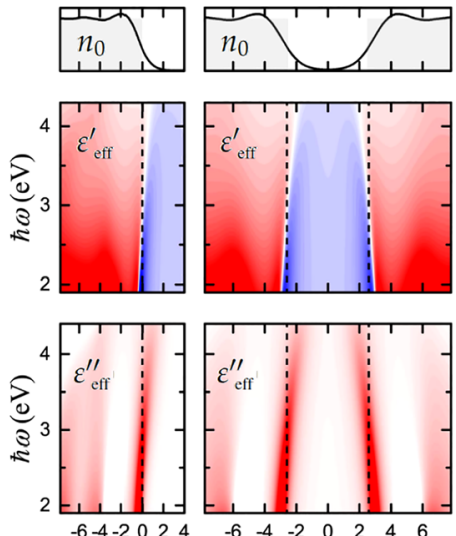

$x(\AA)$ (b)
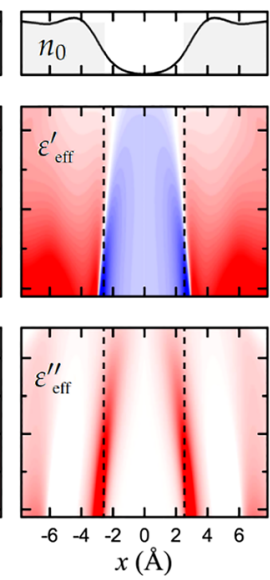

Figure 15: Plot of the spatial and spectral dependence of the real (middle row) and imaginary (lower row) parts of $\varepsilon_{\text {eff }}(\boldsymbol{r}, \omega)=\varepsilon_{\text {eff }}^{\prime}(\boldsymbol{r}, \omega)+i \varepsilon_{\text {eff }}^{\prime \prime}(\boldsymbol{r}, \omega)$ in the near vicinity of the jellium surface. For completeness, the top row illustrates the associated equilibrium electron density $n_{0}(\boldsymbol{r})$. (a) A single semi-infinite jellium surface. (b) Superposition of two independent semi-infinite jellium surfaces, with no inter-surface charge transfer. (c) Two fully coupled jellium surfaces, including the possible inter-surface charge transfer associated with quantum tunneling. Figure adapted from Ref. [244] (C) 2017 World Scientific Publishing Company).

where the nonlocal response is being effectively represented by a spatially in-homogeneous local-response function.

Deep inside the bulk of the jellium, we will naturally recover the bulk dielectric function, while near the surface, the approach should be able to quantitatively reveal the anticipated surface-enhanced Landau damping. Indeed, the lower part of the panel (a) of Figure 15 unambiguously illustrates how locally at the surface, the damping may significantly exceed the bulk part. This is the surface-enhanced Landau damping, that the GNOR model captures through the classical diffusion constant $\mathscr{D}$ [332]. In the context of the microscopic surface-response functions, this enhanced Landau damping is contained in the imaginary part of the Feibelman $d$-parameter, i.e. $d_{\perp}^{\prime \prime}(\omega)$. It should be emphasized that this surface-enhanced Landau damping will occur at any metal surface, whether the surface belongs to a tiny metal nanoparticle or it is the surface of a macroscopic piece of shiny metal. Naturally, its significance for electrodynamics is greatly enhanced in systems with an increased surface-to-volume ratio, while in larger systems the overall damping will be dominated by the bulk contributions. In the next section, we will discuss the implications of surface-enhanced Landau damping for the understanding of the dynamics of dimers with sub-nanometric gaps.

\subsection{Quantum tunneling}

One of the topics that have perhaps sparked the most interest in quantum plasmonics is associated with quantum tunneling [82, 298, 333]. Intuitively, quantum tunneling could potentially play a role in plasmonic dimers with a sub-nanometric gap, which would allow the quantum tunneling of charge across the classically prohibited energy barrier. If so, this should manifest in a CTP even when the particles do not physically kiss each other. Intuitively, the relaxation of the associated tunneling currents should in turn represent also a broadening mechanism, shortcircuiting the capacitive mechanism behind the BDP [82]. The gap-dependent broadening in such nanogap dimers has been explained by the phenomenological quantumcorrected model (QCM) [164] that invokes additional dissipation attributed to the relaxation of tunneling currents.

While obviously successful in qualitatively fitting results of $a b$ initio simulations [164, 201, 236, 252], the QCM approach raises also relevant questions regarding its physical foundation. The understanding of linear-response mesoscopic quantum electron transport [42] is that the tunneling through the classically forbidden gap region is elastic (ballistic transport), while energy relaxation takes place inside the metallic contact regions. We note that this relaxation would eventually have to occur in a co-existence with the relaxation associated with the surface-enhanced Landau damping that we have discussed in the preceding Section 7.3. The above being said inelastic tunneling can of course occur if the electron is driven by a sufficiently large electrical field (large voltage drop across the barrier) that opens an energy window for interactions with other dynamic degrees of freedom such as in photon or phonon-assisted tunneling. As examples, this may manifest in photon emission in direct current (DC) driven gaps in a scanning tunneling microscope (STM) [334] and optical rectification in a plasmonic nanogap [335]. The important new insight brought by ab initio simulations in Refs [202] is that indeed the surface-enhanced Landau damping seems to entirely dominate the optical-frequency dynamics of the plasmonic dimer even deep into the sub-nanometric gap regime, where the low-frequency dynamics is commonly associated with quantum tunneling phenomena. To illustrate this, panels (b) and (c) in Figure 15 revisits the concept of the effective dielectric function, $\varepsilon_{\text {eff }}(r, \omega)$ $=\varepsilon_{\mathrm{eff}}^{\prime}(\boldsymbol{r}, \omega)+i \varepsilon_{\mathrm{eff}}^{\prime \prime}(\boldsymbol{r}, \omega)$, contrasting ab initio results for two coupled jellium surfaces [panel (c)] to the case of the superposition of two independent jellium surfaces of the same kind [panel (b)]. While the former case obviously accounts for the possibility for quantum tunneling currents to be important for the optical-frequency dynamics, 
inter-surface charge transfer is by construction clearly prohibited in the latter case. The very surprising outcome from the space-energy maps of $\varepsilon_{\text {eff }}(r, \omega)$ is that one can hardly tell the differences between the two cases. Even for a gap of only $3 \AA$, the dynamics of the dimer are still dominated by the surface-enhanced Landau damping observed already for the two independent surfaces [202]. Though based on a specific jellium problem, this observation calls into question the alternative quantum tunneling explanations of the gap-dependent broadening in dimers. On the other hand, the very same example illustrates how the plasmon dynamics of the monomer and the dimer seems dominated by some other common physics, being also of a quantum origin: surface-enhanced Landau damping.

Gap-dependent broadening has indeed been observed experimentally, while the experiments have sometimes been interpreted solely as due to quantum tunneling. In particular, seminal studies - "Revealing the quantum regime in tunneling plasmonics" [159] and "Observation of quantum tunneling between two plasmonic nanoparticles" [254] - are clearly alluding to the quantum tunneling as the microscopic mechanism underlining the experimental observations. The optical response has been complimented by low-frequency electrical measurements in the DC tunneling regime [159], but it should, however, be emphasized that there is an absence of explicit electrical measurements of any optical-frequency tunneling currents (being naturally extremely difficult due to limited electrical measurement capabilities in the opticalfrequency regime). As such, these experiments remain important intriguing observations, while perhaps not presenting the smoking gun for optical-frequency quantum tunneling.

Naturally, the classically prohibited energy barrier that separates the two surfaces is closely related to the work function of the metals. Thus, turning to other materials realizations could be one potential way to provide better conditions for more explicit observations of quantum tunneling phenomena at optical frequencies. On the other hand, Landau damping might be influenced as well [264]. Another related approach would be to introduce (molecular) matter into the gap region of the dimer [161, 162, 298], thus effectively providing a stepping stone for charge transfer and the CTP.

The pragmatic question is if charges would have a sufficiently long time to tunnel through the classically prohibited energy barrier before the optical field has its polarity reversed? Acknowledging that the concept of a chargetransfer tunneling time $\tau_{\mathrm{CT}}$ is a delicate topic in itself [336], tunneling would only be important to the overall dimer dynamics of the junction at sufficiently low frequencies where $\omega \tau_{\mathrm{CT}} \ll 1$. This circuit picture was already raised in Ref. [168], providing also some arguments that $\omega \tau_{\mathrm{CT}} \gg 1$ in somewhat comparable STM experiments, but the magnitude of $\tau_{\mathrm{CT}}$ in plasmonic experiments remains an open question.

Proposals favoring the importance of quantum tunneling in dimer dynamics were founded in ab initio treatments of the jellium problem within TDDFT, and the phenomenological QCM was developed from there [164, 252]. As a competing phenomenological model, GNOR instead accounts semiclassically for surface-enhanced Landau damping [168]. So which model is the more relevant one? We have already seen how further jellium considerations offer microscopic support for the dominating role of surfaceenhanced Landau damping for dimers. In fact, GNOR offers a unified description of both monomers and dimers, while the QCM is naturally not applicable to the former. In order to allow experiments to independently settle on the relative importance of quantum tunneling and Landau damping, circular dichroism in nanoparticle helices has recently been proposed as a useful platform [332]. No matter the efforts in finding fruitful materials systems, a more fundamental challenge remains: dynamic tunneling phenomena rely critically on overlapping evanescent tails of electron density reaching beyond the surfaces of the metals, but the very same tails constitute a source of surface response too [271]. As a fundamental consequence of causality and Kramers-Kronig relations [41], this surface response is inevitably associated with surface-enhanced Landau damping.

\subsection{Quantum plasmons in low-dimensional systems}

Plasmons can be hosted in a broad plethora of physical systems, having at least the gas of free electrons in common. Recalling the discussion of the Drude model, Eq. (8), the plasma frequency $\omega_{p} \propto n_{0}^{1 / 2}$ reflects the density $n_{0}$ of the free-electron gas. Beyond the relatively dense 3D electron gas (3DEG) of the metals, plasmons with a lower plasma frequency can also be supported by the more dilute 3DEG of doped semiconductors. Nonlocal effects exist also in this situation, which have been explored in hydrodynamic models [69, 70, 337]. In passing, we note that even undoped semiconductors can host nonlocal phenomena associated with excitons [338], and also interband transitions can resemble plasmonic behavior [74].

Turning the attention to the dimensionality of the electron gas, the advents of semiconductor crystal growth and semiconductor heterostructures paved the way for the $2 \mathrm{D}$ 
electron gas (2DEG) [339]. In addition to many fascinating electronic quantum phenomena, the 2DEG can also host collective plasmonic oscillations, being of an in-plane longitudinal kind with a common $\omega\left(\boldsymbol{q}_{\|}\right) \propto \boldsymbol{q}_{\|}^{1 / 2}$ dispersion relation [340, 341].

With the discovery of atom-thin 2D electron systems, such as doped graphene, there is now a tremendous focus on 2D plasmonics [67, 72]. The following subsections will briefly mention some of those directions. The main theme is here that the more dilute the electron gas, the potentially larger are the intrinsic length scales of the electron gas, such as the Fermi wavelength $\lambda_{\mathrm{F}}$ or the Thomas-Fermi screening length $q_{\mathrm{TF}}^{-1}$. In the context of nonlocal response, the hypothesis is thus that nonlocal effects could manifest at longer length scales than we have so far observed for metals (see Table 1).

The attention to low-dimensional systems, naturally also involves a curiosity for the physics and materials properties that evolve in the transition from 3D materials systems to the true 2D world of atom-thin sheets of materials, such as graphene. Figure 16 illustrates this transitioning, offering now a new paradigm under the headline of transdimensional photonics [342]. The following subsections will discuss particular aspects of low-dimensional materials in connection with the surface-response functions of metal surfaces.

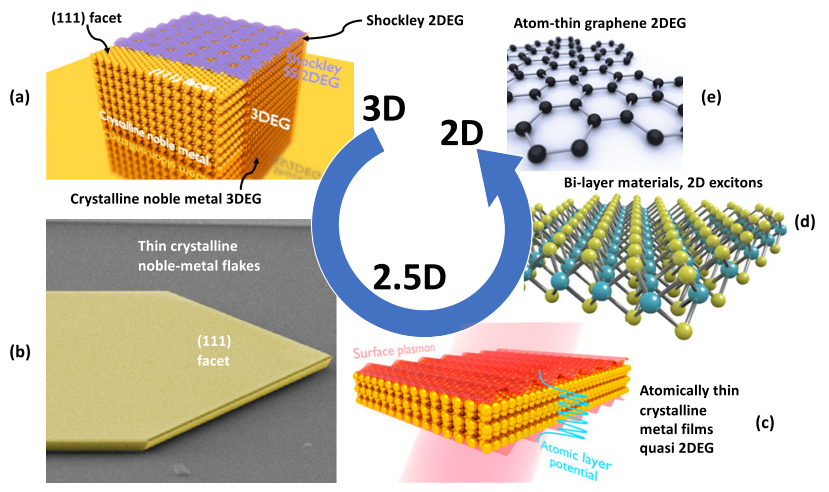

Figure 16: Illustration of the materials paradigm of transdimensional photonics [342], with the electrodynamics spanning the plasmonic response of both the 3DEG and 2DEG systems. Material realizations include (a) 3D crystalline metals, (b) quasi-2D crystalline metal films [320, 343], (c) atom-thin metal films [344, 345], (d) layered 2D exciton materials [32, 346], and eventually (e) one-atom thin 2D materials $[30,67]$. The support of a 2DEG by Shockley surface states, closely analogous to the 2DEG in graphene, is closing the circle of this new emerging materials paradigm. 2DEG, two-dimensional electron gas; 3DEG, three-dimensional electron gas.

\subsubsection{Graphene plasmons}

The optical response of graphene is characterized by a 2D surface conductivity $\sigma_{\|}\left(\boldsymbol{q}_{\|}, \omega\right)$ that exhibits spatial dispersion associated with both the intraband response of the free-electrons associated with doping, as well interband transitions [67], both aspects reflecting the underlying linear electronic dispersion relation [347]. Depending on the level of doping and the ratio of optical energy $\hbar \omega$ relative to the Fermi energy $\mathcal{E}_{F}$, quantum nonlocal effects can manifest in graphene nanostructures of a finite dimension $[73,348-351]$ or when the large- $\boldsymbol{q}_{\|}$response of graphene is probed, e.g. by singular metasurfaces [352], electron beams [353, 354] or by in-elastic scattering processes [355]. In addition, the termination of the graphene lattice can also lead to electronic edge states at the Dirac point, e.g. zigzag-terminated graphene lattices, which can promote additional interband transitions and damping, thus resembling in some aspects the surface-enhanced Landau damping that can occur at metal surfaces due to the termination of the electron gas. However, for graphene lattices, this is an unambiguous manifestation of the underlying atomic lattice and its symmetries. As an example, such edge phenomena can manifest in $R^{-1}$ size-dependent frequency shifts and damping in graphene nanodisks [73] and nanotriangles [349]. In the following, we will focus on the possibilities that graphene can further offer in the context of unraveling mesoscopic electrodynamics at metal surfaces.

Adding 2D materials to the surfaces of metals, the finite $\sigma_{\|}\left(\boldsymbol{q}_{\|}, \omega\right)$ now allows surface currents. As such, an added 2D material can also be represented as a surface-response function, contributing a finite $d_{\|}$-component to the metal surface, initially being characterized by $d_{\|}=0$. Metal particles wrapped in graphene [356] and metal surfaced covered by 2D exciton material [357] represent such examples. Likewise, the placement of graphene in closeness, proximity to a metal mirror has already unveiled intriguing quantum effects [293], arising as an interplay of nonlocal $2 \mathrm{D}$ plasmons in the graphene and their screening by the nearby 3DEG of the metal, responding nonlocally by itself [358]. Since the optical properties of the graphene are in this context remarkably well-established within an analytical RPA theory for $\sigma_{\|}\left(\boldsymbol{q}_{\|}, \omega\right)$, the graphene might be used as a probe that can teach us more about the less known nonlocal properties of the nearby surface, potentially allowing an experimental determination of the Feibelman parameters [295]. As we shall see in the next subsection, this can even be extended to new explorations of atomic-scale properties beyond the jellium picture of metals. Finally, 
the use of graphene as a probe of nearby electrodynamics was recently even suggested as a possible avenue for explorations beyond the common metallic state in a correlated matter [359-361].

\subsubsection{Shockley surface states}

Crystalline metals are intuitively superior to their polycrystalline counterparts [362], and their practical importance for plasmonics [363] should not be underestimated [287,343, 364-368]. At the same time, it is well-established in surface science [369] that (111) noble-metal surfaces have Shockley states [370] (occasionally also referred to as Tamm states [371]) with a 2DEG, see panel (a) in Figure 16, with no counterparts for other surface facets (or polycrystalline films for that sake). The associated 2DEG is in itself fundamentally interesting, somewhat resembling the 2DEG of graphene [see panel (e) of Figure 16], while exhibiting a free-electron like parabolic dispersion.

Crystalline noble-metal particles can exhibit many different facets [372], being reflected also in morphologydependent resonances [373, 374]. On the other hand, larger planar flakes now gaining attention in plasmonics are characterized in particular by the (111) surfaces, see panel (b) in Figure 16, while the flake edges can naturally be of other kinds too [343]. The (111) facet of crystalline flakes is now revitalizing the interest in Shockley surface states also in the context of plasmonics.

The existence of a Shockley 2DEG stimulates explorations in the spirit of 2D graphene plasmons [375], as we have discussed in the preceding Section 7.5.1. Angleresolved electron energy loss spectroscopy has revealed 2D plasmons associated with similar surfaces [376, 377], but with low energy and large wave vectors, rendering them even more confined to the surface than what is seen for the confinement of graphene plasmons to the carbon monolayer [378]. Similarities with graphene plasmonics might actually come in handy, with graphene plasmons themselves providing the means to probe and unveil 2DEG (and 3DEG surface) quantum phenomena. As such, the approach of Lundeberg et al. [293] could be extended to explore an arising interplay of nonlocal 2D plasmons (of both the graphene and the Shockley-type kind) and their screening by the nearby $3 \mathrm{DEG}$, responding nonlocally itself [291, 358]. While remarkable recent experiments exploited poly-crystalline metal films [293, 379], the (111) surfaces might offer a new paradigm when interfaced with graphene. Hybridization of graphene and Shockley-type plasmons would open new avenues for explorations of quantum effects in highly confined electron gasses.
In the context of the plasmonic response of the bulk 3DEG, the surface-response functions are now dressed by the presence of also the Shockley 2DEG [328], potentially rendering $d_{\|} \neq 0$ for (111) noble-metal surfaces, while $d_{\|}$ $=0$ for any other surface facet [292]. The curious question is if there are any plasmonic signatures that would distinguish (111) surfaces [380, 381] from any other surface of the same metal?

Finally, turning to few-atom thin films, out-of-plane quantum confinement should render the 3DEG and its plasmons 2D-like [382]. Transition to this regime is extremely interesting [344, 345], being at the heart of transdimensional photonics [342]. In such situations, with less clear ways to distinguish the surface from bulk, one would perhaps have to return to the selvage considerations of Sipe [262].

\subsubsection{Molecular plasmons}

Through doping, macro-molecules can be made conductive, thus potentially also supporting a plasmonic response that could co-exist with single-particle optical transitions in the molecule [383]. In particular, polycyclic aromatic hydrocarbons are planar macro-molecules that in many ways resemble finite-size nanoflakes of graphene. When doped by the addition of few electrons, the observed strong optical response has been given a plasmonic interpretation [384]. The important question is of course how to unambiguously reveal the plasmonic nature and how to tell it apart from common single-particle transitions? As such, the microscopic definition of a plasmon that engages only very few electrons is yet to be fully understood. How plasmon-like is the excitation? Theoretical efforts have been made to quantify the degree of collectiveness by introducing a so-called plasmonicity measure $[385,386]$. Since plasmons in atom-thin planar systems are in-plane pressure waves of a longitudinal kind, this question of plasmonicity suddenly connects to the work of also nonlocal plasmonics in metals, where the electromagnetic energy of the plasmon can be hosted in both transverse and longitudinal degrees of freedom (the former being accounted for by classical formalism, while the latter emerges from quantum considerations).

Using RPA techniques and energy-density arguments there is a rigorous way to estimate the relative fraction of electromagnetic energy hosted by longitudinal degrees of freedom [174]. Interestingly, this ratio can be expressed also in terms of experimentally measurable quantities, e.g. the nonclassical-impact parameter (NCI) [174]

$$
\mathrm{NCI}=1+\frac{\operatorname{Im}\left\{\varepsilon^{-1}\right\}}{Q}, \quad 0<\mathrm{NCI}<1,
$$


where $\operatorname{Im}\left\{\varepsilon^{-1}\right\}$ is the electron-energy loss function (e.g. being probed in EELS experiments [96]) and $Q=\omega / \Delta \omega$ is the quality factor of the resonance. By construction, $\mathrm{NCI}=0$ for entirely classical (transverse) electrodynamics, while NCI = 1 for fully developed nonclassical (longitudinal) electrodynamics. This was already used to access the degree of quantumness (nonlocality) associated with the EELS observation of frequency blueshifts in silver nanoparticles [124, 138].

Quite unintentionally, the NCI concept can equally well be used as a rigorous plasmonicity measure for molecular plasmonics in planar macro-molecules [386]: A vanishing NCI ratio for electrodynamics entirely associated with transverse single-particle transitions in the molecule, while the ratio attains the value of unity for longitudinal plasmon-like excitations. More recent works have developed further on this energy concept in molecular plasmonics [387, 388].

\subsection{Surface chemistry}

The very fact that the formalism of surface-response functions was emerging from the surface-science community, naturally also suggests the potential usefulness not only to the more fundamental explorations of physics and electrodynamics but perhaps also to surface chemistry and applications areas such as photocatalysis. Localized plasmon resonance is confined to the surfaces and surface chemistry can thus change the plasmon resonance frequency, which is essentially the principle behind plasmonic sensing [389-391]. Likewise, already early experiments revealed a sensitivity of the plasmon damping to the chemical nature of the nanoparticle interface [392]. In the context of Refs. $[122,202]$ and the introduction of Feibelman parameters [80], this can be linked to the microscopic surface-response functions (here predominantly $d_{\perp}^{\prime \prime}$ ). Another curious point relates to Eq. (35) where $x_{0}$ coincides with the static imageplane position that emerges from a self-consistent solution of the jellium perturbed by a static field [393-395]. As such, $x_{0}$ is being a quantity of interest in surface science at large and a particular example being that of the surface-assisted van der Waals interaction of an atom near a metallic surface $[266,394]$.

Later, surface chemistry was explored as a means for reversing the size-dependence of the SPR frequency [396], thus illustrating how the chemical nature of the surface can alter even the sign of $d_{\perp}^{\prime}$. In very recent experiments [397], the formation of surface layers with in-plane longerrange order may in principle even facilitate the support of surface currents, thus being related to also $d_{\|}$. In support of this speculation, we have already discussed above how the addition of 2D materials to the surface would effectively add a finite $d_{\|}$component to the metal surface, initially being characterized by $d_{\|}=0$.

Surface-enhanced Landau damping is an inevitable aspect of the strong confinement of light through SPs [243]. While being a curse for many applications, it is also a blessing for the funneling of energy to the very nanoscale through the local dissipation of electromagnetic energy. This local plasmonic heating [398] has facilitated numerous applications, ranging from cancer treatment [399] to re-configuration of morphology-dependent plasmonic resonators-[400], while the associated hot electrons hold potential for additional use [401].

From the Newns-Anderson model [402], the chemical binding of atoms and molecules to the surface is well understood in terms of hybridization of surface states with the add-atoms, where the equilibrium density profile naturally plays some role. The curious question is if the quantum spill-out of the optically induced surface-charge density could participate in surface chemistry in a similar way? The prospects for this would very much depend on chemical-reaction rates and the possible charge-transfer rate $\tau_{\mathrm{CT}}$ relative to the driving optical frequency $\omega$. Interestingly, this speculation is not entirely unrelated to the discussions of quantum tunneling in Section 7.4.

\subsection{Quantum plasmonics}

With the focus on the quantum and nonlocal aspects of plasmons, the mesoscopic electrodynamics formalism forms an integral part of the rapidly developing field of quantum plasmonics $[4,6,7,403]$ embracing both the fundamental research and the quantum technology perspectives of plasmonics in the quantum regime. Most importantly, this intriguing development can involve the quantum aspects not only in at least two different ways: (i) the possible quantum and nonlocal aspects of the matter supporting the plasmons but also (ii) the quantum aspects of the light fields, i.e. nanoscale quantum optics involving plasmons [404-415]. The regime where both light and matter exhibits quantum dynamics has prospects for emerging quantum technology [8], including the unique opportunity for quantum electrodynamics (QED) with plasmonics [413] and plasmon-enhanced generation of nonclassical light [10]. Plasmon-emitter interactions are of paramount importance to the development of quantum plasmonics, including the engineering of single-photon emission dynamics [9, 416-418]. As examples, individual quantum emitters have been coupled to propagating plasmons [419-422] and to localized gap plasmons [423-428]. The interactions are generally maximal at short emitter-surface separations, 
with emission being quenched by coupling to nonradiative degrees of freedom when getting closer to the surface [429, 430]. When the separation $h$ enters the $10-20 \mathrm{~nm}$ regime, classical theory (valid when $h \gg d_{\perp}$ ) progressively deteriorates due to its neglect of quantum and nonlocal effects. In passing, we note that mesoscopic corrections to the pointdipole approximation can also occur if dimensions of the emitter itself cannot be neglected on the scale of $h$ [431]. Accounting for the surface-response functions of the metal, surface-enhanced Landau damping is shown to have a dramatic impact on the amplitude and spectral distribution of plasmon-emitter interactions [296]. Similar nonlocal effects have also been explored in the context of spontaneous emission rates and plasmon-enhanced molecular fluorescence [432-434] as well as strong coupling between excitons or quantum emitters and plasmons [435-438].

Turning to more general plasmonic nanostructures, quasi-normal mode (QNM) formalism for open-resonator problems [439, 440] represents an important step for the computational treatment of plasmon-enhanced singlephoton emission and for further exploitation of QED with plasmonic systems [441]. Here, nonlocal hydrodynamic aspects have been included in QNM formalism, thus allowing the first computational explorations of the quantum aspects of both light and matter in QED problems [442]. The representation of the surface-response functions through mesoscopic boundary conditions can likewise be used for quantum-corrected QNM formalism. This forms also a convenient starting point for evaluations of spectral shifts and broadening in the presence of retardation effects [269] (see also Ref. [443] for an eigenvalue approach), thus going beyond the quasi-static considerations of Ref. [122].

\subsection{Metamaterials}

While this review has its focus on the nonlocal response associated with a quantum electron gas, it should be mentioned that there is a parallel interest in nonlocal effects and spatial dispersion that manifest in artificial periodic media and metamaterials [444], including Refs. [445-453]. Quoting Podolskiy from the discussions at the 2015 Faraday Discussions on nanoplasmonics [331]: "The drastic difference between nonlocality in homogeneous media studied previously and nonlocality in metamaterials lies in the origin of additional waves. In homogeneous materials, the nonlocal response is attributed to the spatial dispersion of the material itself. In metamaterials, however, nonlocality appears at the "effective medium" level; the response of every component of the metamaterial may remain local, while the granularity of the composite leads to the spatial dispersion of the effective permittivity."
Nevertheless, studies of nonlocal plasmonic effects in metal-based hyperbolic metamaterials [117, 454], plasmonic crystals [455], and epsilon-near-zero phenomena [456] constitute examples of the relevance of nonlocal plasmonic response to also concepts in metamaterials research. Likewise, the nonlocal response of metamaterials also has implications for SPPs sustained at their surfaces [457]. While clearly going beyond the focus of this review, it is an interesting open question to which extend metamaterials governed by classical electrodynamics would be able to mimic the spatial dispersion and nonlocal phenomena beyond LRA aspects of the SP [458-460].

\subsection{Topological nanophotonics}

Let us finally turn to the quickly developing area of topological phenomena, which are ubiquitous in physics and spanning areas from condensed-matter physics to physical cosmology. Also, electrodynamics is now harvesting from topology in many exciting ways with topological photonics [461] manifesting in a plethora of novel directions [35, 38,39 ] that challenge the common understanding of light propagation and light-matter interactions. In bridging topological physics (understood in broad terms, including also nonreciprocity and non-Hermitian systems) and nanophotonics, unforeseen topological control of quantum light-matter interactions can be anticipated [40], with developments in topological nanophotonics facilitating intriguing interplays of light, matter, topology, and symmetry. Naturally, plasmons also play a potential future role in such adventures and as such, the quantum and nonlocal plasmonics might become an important aspect of this development too. As a recent example of this, the nonlocal response of plasmons is central to discussions [462-464] of surface-magneto-plasmons [465-467] and the prospects for optical isolators and nonreciprocal plasmonics $[468,469]$.

\section{Concluding remarks}

The interaction of light with matter is largely being understood within the framework of classical electrodynamics, linear-response theory, and the LRA. This framework has been instrumental for the interpretation of fundamental experiments, while also fueling new thoughts and numerous predictions of new intriguing electrodynamic phenomena, that have in turn been confronted and confirmed experimentally. The general success of this framework in optical explorations of plasmons can largely be attributed to the relatively "large" dimensions of the metal structures 
hosting the plasmons, thus justifying the LRA. However, the increasing experimental ability to both realize and probe plasmons in yet smaller metallic nanostructures has also disclosed a need for more refined descriptions of the light-matter interactions, possibly including effects associated with the underlying quantum dynamics of the electron gas of the metals. This is the reason for the curiosity and the motivation for the theoretical efforts and concept developments that have led to the mesoscopic electrodynamics framework.

The review summarizes formalism and concept developments that are foundational to mesoscopic electrodynamics and the understanding of quantum and nonlocal plasmon response in metallic nanostructures. The accounts for quantum effects enter the electrodynamics at various levels of complexity, ranging from semiclassical nonlocal corrections of Ohm's law to Feibelman's surface-response functions, that account for the microscopic temporospatial dynamics of the quantum electron gas that manifest at length scales associated with the underlying atomic lattice. In all cases, there is an effort to offer physically transparent directions for the implementation of quantum corrections in classical electrodynamics, thus also offering practical solutions that can be immediately implemented in state-of-the-art computational electrodynamics, already widely exploited in the research and engineering communities.

The dominating and unifying quantum effects that manifest in both individual monomers and dimers (formed from pairs of such monomers) can be briefly summarized as

- Nonlocal response associated with the finite compressibility of the quantum electron gas, causing sizedependent spectral shifts of plasmon resonances,

- Landau damping and spill-out associated with the surface termination of the quantum electron gas, causing size-dependent spectral shifts and broadening.

While clearly acknowledging the importance of quantum spill-out of charge, it is argued that relaxation related to quantum-tunneling-mediated charge transfer perhaps represents a less significant broadening contribution in dimer structures as compared to spill-out related surfaceenhanced Landau damping. Admitted, this conclusion is challenged by seemingly widespread intuition and consensus in the field of plasmonics. The above paradigm is supported by $a b$ initio TDDFT calculations while being somewhat in opposition to the competing paradigm of quantum tunneling in nanogap plasmonic structures. Noteworthy, the competing paradigms are both rooted in the same - highly idealized - jellium-model considerations and a few atomistic $a b$ initio atomistic descriptions [288, 289], while experimental realizations can only be anticipated to be far more complex. Ideally, future fundamental experiments would be designed with an emphasis on confronting and clarifying these competing interpretations. Perhaps, both interpretations may coexist without actually competing.

Beyond the fundamental interest in the quantum and nonlocal response of plasmons in metallic nanostructures, the mesoscopic electrodynamics framework is also central to emerging quantum technology that harvests from plasmon-enhanced light-matter interactions. In particular, nonlocal response and surface-enhanced Landau damping have a profound influence on the temporal dynamics of quantum emitters placed in the near vicinity of metal nanostructures.

The new insight in the quantum and nonlocal response of plasmons is potentially also of relevance for future explorations now heading in a number of exciting directions, ranging from the fundamentally important experimental probing of surface-response functions - with the aid of surface-added atom-thin 2D materials - to the exploitation of light-induced hot electrons in surface chemistry.

In closing, the timing of this review follows the recent 200 years anniversary of Ørsted's discovery in electromagnetism. For two centuries fundamental explorations and engineering efforts have advanced enormously on the foundations of classical electrodynamics, while entirely transforming our society and means for human interactions. We are now on the verge of a second era, facing new paradigms in quantum light-matter interactions with prospects for quantum-information processing. The quantum-corrected mesoscopic framework will support such new adventures with plasmons.

Acknowledgment: The mesoscopic electrodynamic formalism reviewed in this paper stands on the shoulders of outstanding young scholars, including Søren Raza [470], Giuseppe Toscano [471], Thomas Christensen [472], and André Gonçalves [473].

For stimulating discussions and collaborations I also acknowledge Martijn Wubs, Antti-Pekka Jauho, Sergey Bozhevolnyi, Kristian Thygesen, Christos Tserkezis, Wei Yan, Christian Wolff, Joel Cox, Marin Soljačić, Javier García de Abajo, Peter Nordlander, Naomi Halas, Javier Aizpurua, Jacob Khurgin, Alexander Uskov, Uriel Levy, Jennifer Dionne, Jeremy Baumberg, Kurt Busch, Carsten Rockstuhl, Nuno Peres, Thomas Søndergaard, M. Ichikawa, John Pendry, Yu Luo, and Antonio Fernández-Domínguez. 
Likewise, I am thankful for the stimulating input from experimental collaborators, including Nicolas Stenger, Sanshui Xiao, Zhaowei Liu, Joel Yang, Jer-shing Huang, Bert Hecht, and Frank Koppens. I also thank Thomas Pertsch for hosting me during my Abbe professorship at the University of Jena - many of the reflections developed during my 2015 guest-lecture series have found their way to this review. The paper is largely based on a recent rehabilitation thesis [474], that was reviewed by Luis Martín-Moreno, George Hanson, and Anders Sørensen - I sincerely thank the committee for the many insightful comments and suggestions. Finally, I am grateful to two anonymous peerreviewers, who provided high-quality feedback that served to further improve the paper in several important ways.

Author contribution: The author has accepted responsibility for the entire content of this submitted manuscript and approved submission.

Research funding: This work is supported by the VILLUM Foundation and the VILLUM Investigator program (grant No. 16498), the Danish National Research Foundation (CNG, project No. DNRF103), the Independent Research Fund Denmark (Elite Researcher Prize, grant No. 7079-00043B), and the Danish Council for Independent Research (Natural Sciences, grant No. 12-133429).

Conflict of interest statement: The authors declare no conflicts of interest regarding this article.

\section{References}

[1] W. H. Zurek, "Decoherence and the transition from quantum to classical," Phys. Today, vol. 44, no. 10, p. 36, 1991.

[2] J. P. Dowling and G. J. Milburn, "Quantum technology: the second quantum revolution," Philos. Trans. R. Soc. A, vol. 361, pp. 1655-1674, 2003.

[3] M. Riedel, M. Kovacs, P. Zoller, J. Mlynek, and T. Calarco, “Europe's quantum flagship initiative," Quantum Sci. Technol., vol. 4, no. 2, p. 020501, 2019.

[4] M. S. Tame, K. R. McEnery, Ş. K. Özdemir, J. Lee, S. A. Maier, and M. S. Kim, "Quantum plasmonics," Nat. Phys., vol. 9, no. 6, pp. 329-340, 2013.

[5] S. I. Bozhevolnyi, L. Martín-Moreno, and F. J. García-Vidal, "Quantum plasmonics," in Springer Series in Solid-State Sciences, vol. 185, Berlin, Springer International Publishing, 2017, pp. i-xiv.

[6] J. M. Fitzgerald, P. Narang, R. V. Craster, S. A. Maier, and V. Giannini, "Quantum plasmonics," Proc. IEEE, vol. 104, no. 12, pp. 2307-2322, 2016.

[7] Z.-K. Zhou, J. Liu, Y. Bao, et al., "Quantum plasmonics get applied,” Prog. Quant. Electron., vol. 65, pp.1-20, 2019.

[8] S. I. Bozhevolnyi and N. A. Mortensen, "Plasmonics for emerging quantum technologies," Nanophotonics, vol. 6, p. 1185, 2017.
[9] S. I. Bozhevolnyi and J. B. Khurgin, "The case for quantum plasmonics,” Nat. Photonics, vol. 11, no. 7, pp. 398-400, 2017.

[10] A. I. Fernández-Domínguez, S. I. Bozhevolnyi, and N. A. Mortensen, "Plasmon-enhanced generation of nonclassical light," ACS Photonics, vol. 5, no. 9, pp. 3447-3451, 2018.

[11] A. Chodos, "July 1820: Oersted and electromagnetism," APS News, vol. 17, no. 7, p. 2, 2008.

[12] R. T. G., "Oersted - the discoverer of electro magnetism," Nature, vol. 107, pp. 492-495, 1921.

[13] B. Mahon, "How Maxwell's equations came to light," Nat. Photonics, vol. 9, no. 1, pp. 2-4, 2015.

[14] J. D. Jackson, Classical Electrodynamics, Hoboken, New Jersey, Wiley \& Sons, 1998.

[15] J. Gertner, The Idea Factory: Bell Labs and the Great Age of American Innovation, New York, Penguin Publishing Group, 2012.

[16] N. Engheta, “150 years of Maxwell's equations," Science, vol. 349, no. 6244, pp. 136-137, 2015.

[17] C. K. Kao, "Nobel lecture: sand from centuries past: send future voices fast," Rev. Mod. Phys., vol. 82, pp. 2299-2303, 2010.

[18] J. D. Joannopoulos, P. R. Villeneuve, and S. H. Fan, "Photonic crystals: putting a new twist on light," Nature, vol. 386, no. 6621, pp. 143-149, 1997.

[19] J. C. Knight, "Photonic crystal fibres," Nature, vol. 424, no. 6950, pp. 847-851, 2003.

[20] H. Chen, C. T. Chan, and P. Sheng, "Transformation optics and metamaterials," Nat. Mater., vol. 9, no. 5, pp. 387-396, 2010.

[21] A. Poddubny, I. Iorsh, P. Belov, and Y. Kivshar, "Hyperbolic metamaterials," Nat. Photonics, vol. 7, no. 12, pp. 948-957, 2013.

[22] M. Kadic, G. W. Milton, M. van Hecke, and M. Wegener, “3D metamaterials," Nat. Rev. Phys., vol. 1, pp. 198-210, 2019.

[23] A. V. Kildishev, A. Boltasseva, and V. M. Shalaev, "Planar photonics with metasurfaces," Science, vol. 339, no. 6125, p. 1232009, 2013.

[24] F. Ding, A. Pors, and S. I. Bozhevolnyi, "Gradient metasurfaces: a review of fundamentals and applications," Rep. Prog. Phys., vol. 81, no. 2, p. 026401, 2018.

[25] I. Liberal and N. Engheta, "Near-zero refractive index photonics," Nat. Photonics, vol. 11, no. 3, pp. 149-158, 2017.

[26] I. Liberal and N. Engheta, "Zero-index structures as an alternative platform for quantum optics," Proc. Natl. Acad. Sci. U.S.A., vol. 114, no. 5, pp. 822-827, 2017.

[27] N. Kinsey, C. Devault, A. Boltasseva, and V. M. Shalaev, "Near-zero-index materials for photonics," Nat. Rev. Mater., vol. 4, pp. 742-760, 2019.

[28] F. Xia, H. Wang, D. Xiao, M. Dubey, and A. Ramasubramaniam, "Two-dimensional material nanophotonics," Nat. Photonics, vol. 8, no. 12, pp. 899-907, 2014.

[29] T. Low, A. Chaves, J. D. Caldwell, et al., "Polaritons in layered two-dimensional materials,” Nat. Mater., vol. 16, no. 2, pp. 182-194, 2017.

[30] S. Xiao, X. Zhu, B.-H. Li, and N. A. Mortensen, "Graphene-plasmon polaritons: from fundamental properties 
to potential applications," Front. Physiol., vol. 11, p. 117801, 2016.

[31] D. N. Basov, M. M. Fogler, and F. J. García de Abajo, "Polaritons in van der Waals materials," Science, vol. 354, p. aag1992, 2016.

[32] P. A. D. Gonçalves, N. Stenger, J. D. Cox, N. A. Mortensen, and S. Xiao, "Strong light-matter interactions enabled by polaritons in atomically-thin materials," Adv. Opt. Mater., vol. 8, p. 1901473, 2020.

[33] C. Tserkezis, A. I. Fernández-Domínguez, P. A. D. Gonçalves, et al., "On the applicability of quantum-optical concepts in strong-coupling nanophotonics," Rep. Prog. Phys., vol. 83, no. 8, p. $082401,2020$.

[34] A. Reserbat-Plantey, I. Epstein, I. Torre, et al., "Quantum nanophotonics in two-dimensional materials," ACS Photonics, vol. 8, no. 1, pp. 85-101, 2021.

[35] R. El-Ganainy, K. G. Makris, M. Khajavikhan, Z. H. Musslimani, S. Rotter, and D. N. Christodoulides, "Non-Hermitian physics and PT symmetry," Nat. Phys., vol. 14, no. 1, pp.11-19, 2018.

[36] S. K. Özdemir, S. Rotter, F. Nori, and L. Yang, "Parity-time symmetry and exceptional points in photonics," Nat. Mater., vol. 18, no. 8, pp. 783-798, 2019.

[37] M.-A. Miri and A. Alù, "Exceptional points in optics and photonics," Science, vol. 363, no. 6422, p. eaar7709, 2019.

[38] A. B. Khanikaev and G. Shvets, "Two-dimensional topological photonics," Nat. Photonics, vol. 11, no. 12, pp. 763-773, 2017.

[39] T. Ozawa, H. M. Price, A. Amo, et al., "Topological photonics,” Rev. Mod. Phys., vol. 91, p. 015006, 2019.

[40] N. A. Mortensen, S. I. Bozhevolnyi, and A. Alù, "Topological nanophotonics," Nanophotonics, vol. 8, p. 1315, 2019.

[41] T. Dethe, H. Gill, D. Green, et al., "Causality and dispersion relations,” Am. J. Phys., vol. 87, no. 4, pp. 279-290, 2019.

[42] S. Datta, Electronic Transport in Mesoscopic Systems, Cambridge, Cambridge University Press, 1995.

[43] O. Keller, Quantum Theory of Near-Field Electrodynamics, Berlin, Springer-Verlag, 2011.

[44] M. Wubs and N. A. Mortensen, "Nonlocal response in plasmonic nanostructures," in Springer Series in Solid-State Sciences, vol. 185, Berlin, Springer International Publishing, 2016, pp. 279-302.

[45] P. B. Johnson and R. W. Christy, "Optical constants of the noble metals,” Phys. Rev. B, vol. 6, pp. 4370-4379, 1972.

[46] L. Novotny and B. Hecht, Principles of Nano-Optics, Cambridge, Cambridge University Press, 2012.

[47] U. Hohenester, Nano and Quantum Optics, Berlin, Springer International Publishing, 2020.

[48] A. Maradudin, J. R. Sambles, and W. L. Barnes, "Modern plasmonics," in Handbook of Surface Science, North-Holland, 2014.

[49] F. Bloch, "Bremsvermögen von Atomen mit mehreren Elektronen,” Z. Phys., vol. 81, pp. 363-376, 1933.

[50] H. Smith and H. Højgaard Jensen, Transport Phenomena, Oxford, Oxford University Press, 1989.

[51] H. Bruus and K. Flensberg, Many-Body Quantum Theory in Condensed Matter Physics: An Introduction, Oxford, Oxford University Press, 2014.
[52] J. Lindhard, "On the properties of a gas of charged particles," Kgl. Danske Videnskab. Selskab Mat.-Fys. Medd., vol. 28, pp. 1-57, 1954.

[53] N. D. Mermin, "Lindhard dielectric function in the relaxation-time approximation," Phys. Rev. B, vol. 1 , pp. 2362-2363, 1970.

[54] L. Tonks and I. Langmuir, "Oscillations in ionized gases," Phys. Rev., vol. 33, pp. 195-210, 1929.

[55] D. Bohm and D. Pines, "A collective description of electron interactions. I. magnetic interactions," Phys. Rev., vol. 82, pp. 625-634, 1951.

[56] D. Pines and D. Bohm, "A collective description of electron interactions: II. Collective vs individual particle aspects of the interactions," Phys. Rev., vol. 85, pp. 338-353, 1952.

[57] D. Bohm and D. Pines, "A collective description of electron interactions: III. Coulomb interactions in a degenerate electron gas," Phys. Rev., vol. 92, pp. 609-625, 1953.

[58] D. Pines, "A collective description of electron interactions: IV. Electron interaction in metals," Phys. Rev., vol. 92, pp. 626-636, 1953.

[59] D. Pines, "Collective energy losses in solids," Rev. Mod. Phys., vol. 28, pp. 184-198, 1956.

[60] J. M. Pitarke, V. M. Silkin, E. V. Chulkov, and P. M. Echenique, "Theory of surface plasmons and surface plasmon polaritons," Rep. Prog. Phys., vol. 70, pp. 1-87, 2007.

[61] R. H. Ritchie, "Plasma losses by fast electrons in thin films," Phys. Rev., vol. 106, pp. 874-881, 1957.

[62] C. J. Powell and J. B. Swan, "Origin of the characteristic electron energy losses in aluminum," Phys. Rev., vol. 115, pp. 869-875, 1959.

[63] S. Lal, S. Link, and N. J. Halas, "Nano-optics from sensing to waveguiding,” Nat. Photonics, vol. 1, pp. 641-648, 2007.

[64] M. I. Stockman, "Nanoplasmonics: the physics behind the applications," Phys. Today, vol. 64, no. 2, p. 39, 2011.

[65] A. I. Fernández-Domínguez, F. J. García-Vidal, and L. Martín-Moreno, “Unrelenting plasmons," Nat. Photonics, vol. 11, no. 1, pp. 8-10, 2017.

[66] S. A. Maier, Plasmonics: Fundamentals and Applications, Berlin, Springer-Verlag, 2007.

[67] P. A. D. Gonçalves and N. M. R. Peres, An Introduction to Graphene Plasmonics, Singapore, World Scientific, 2016.

[68] N. A. Mortensen, P. Berini, U. Levy, and S. I. Bozhevolnyi, "Proceedings of 9th international conference on surface plasmon photonics (SPP9)," Nanophotonics, vol. 9, p. 244, 2020.

[69] J. R. Maack, N. A. Mortensen, and M. Wubs, "Size-dependent nonlocal effects in plasmonic semiconductor particles," Europhys. Lett., vol. 119, no. 1, p. 17003, 2017.

[70] J. R. Maack, N. A. Mortensen, and M. Wubs, "Two-fluid hydrodynamic model for semiconductors," Phys. Rev. B, vol. 97, p. 115415, 2018.

[71] A. Vasanelli, S. Huppert, A. Haky, T. Laurent, Y. Todorov, and C. Sirtori, "Semiconductor quantum plasmonics," Phys. Rev. Lett., vol. 125, p. 187401, 2020.

[72] F. J. García de Abajo, "Graphene plasmonics: challenges and opportunities," ACS Photonics, vol. 1, no. 3, pp. 135-152, 2014. 
[73] T. Christensen, W. Wang, A.-P. Jauho, M. Wubs, and N. A. Mortensen, "Classical and quantum plasmonics in graphene nanodisks: role of edge states," Phys. Rev. B, vol. 90, p. 241414(R), 2014.

[74] Z. Dong, T. Wang, X. Chi, et al., "Ultraviolet interband plasmonics with Si nanostructures," Nano Lett., vol. 19, no. 11, pp. 8040-8048, 2019.

[75] C. R. Gubbin and S. De Liberato, "Optical nonlocality in polar dielectrics,” Phys. Rev. X, vol. 10, p. 021027, 2020.

[76] G. Barton, "Some surface effects in the hydrodynamic model of metals," Rep. Prog. Phys., vol. 42, no. 6, pp. 963-1016, 1979.

[77] H. Bruus, Theoretical Microfluidics, Oxford, Oxford University Press, 2008.

[78] R. Kubo, "The fluctuation-dissipation theorem," Rep. Prog. Phys., vol. 29, no. 1, p. 255, 1966.

[79] E. Runge and E. K. U. Gross, "Density-functional theory for time-dependent systems,” Phys. Rev. Lett., vol. 52, pp. 997-1000, 1984.

[80] P. J. Feibelman, "Surface electromagnetic-fields," Prog. Surf. Sci., vol. 12, no. 4, pp. 287-407, 1982.

[81] J. Joannopoulos, S. Johnson, J. Winn, and R. Meade, Photonic Crystals: Molding the Flow of Light, 2nd ed., Princeton, Princeton University Press, 2008.

[82] W. Zhu, R. Esteban, A. G. Borisov, et al., "Quantum mechanical effects in plasmonic structures with subnanometre gaps," Nat. Commun., vol. 7, p. 11495, 2016.

[83] N. Rivera and I. Kaminer, "Light-matter interactions with photonic quasiparticles," Nat. Rev. Phys., vol. 2, pp. 538-561, 2020.

[84] P. Dombi, Z. Pápa, J. Vogelsang, et al., "Strong-field nano-optics," Rev. Mod. Phys., vol. 92, p. 025003, 2020.

[85] P. Drude, "Zur elektronentheorie der metalle," Ann. Phys., vol. 306, no. 3, pp. 566-613, 1900.

[86] N. D. Ashcroft and N. D. Mermin, Solid State Physics, Philadelphia, Saunders College Publishing, 1976.

[87] W. J. Ding, J. Z. J. Lim, H. T. B. Do, et al., "Particle simulation of plasmons,” Nanophotonics, vol. 9, no. 10, pp. 3303-3313, 2020.

[88] H. T. B. Do, D. Wen Jun, Z. Mahfoud, W. Lin, and M. Bosman, “Electron dynamics in plasmons," Nanoscale, vol. vol. 13, pp. 2801-2810, 2021.

[89] R. C. Jin, Y. W. Cao, C. A. Mirkin, K. L. Kelly, G. C. Schatz, and J. G. Zheng, "Photoinduced conversion of silver nanospheres to nanoprisms," Science, vol. 294, no. 5548, pp. 1901-1903, 2001.

[90] M. Z. Liu and P. Guyot-Sionnest, "Synthesis and optical characterization of $\mathrm{Au} / \mathrm{Ag}$ core/shell nanorods,"J. Phys. Chem. B, vol. 108, no. 19, pp. 5882-5888, 2004.

[91] A. Ruivo, C. Gomes, A. Lima, et al., "Gold nanoparticles in ancient and contemporary ruby glass," J. Cult. Herit., vol. 9, pp. e134-e137, 2008.

[92] P. Colomban, "The use of metal nanoparticles to produce yellow, red and iridescent colour, from bronze age to present times in lustre pottery and glass: solid state chemistry, spectroscopy and nanostructure,"J. Nano Res., vol. 8, pp. 109-132, 2009.
[93] A. Kristensen, J. K. W. Yang, S. I. Bozhevolnyi, et al., "Plasmonic colour generation," Nat. Rev. Mater., vol. 2, p. 16088, 2017.

[94] D. S. Rezaei, Z. Dong, J. You En Chan, et al., "Nanophotonic structural colors,” ACS Photonics, vol. 8, no. 1, pp.18-33, 2021.

[95] E. Abbe, "Ueber einen neuen Beleuchtungsapparat am Mikroskop,” Arch. Mikrosk. Anat., vol. 9, no. 1, pp. 469-480, 1873.

[96] F. J. García de Abajo, "Optical excitations in electron microscopy,” Rev. Mod. Phys., vol. 82, pp. 209-275, 2010.

[97] A. Polman, M. Kociak, and F. J. García de Abajo, “Electron-beam spectroscopy for nanophotonics," Nat. Mater., vol. 18, pp. 1158-1171, 2019.

[98] N. Nicoletti, M. Wubs, N. A. Mortensen, W. Sigle, P. A. van Aken, and P. A. Midgley, "Surface plasmon modes of a single silver nanorod: an electron energy loss study," Opt. Express, vol. 19, no. 16, pp. 15371-15379, 2011.

[99] J. Henzie, J. Lee, M. H. Lee, W. Hasan, and T. W. Odom, "Nanofabrication of plasmonic structures," Annu. Rev. Phys. Chem., vol. 60, no. 1, pp. 147-165, 2009.

[100] P. Ginzburg and A. V. Zayats, "Localized surface plasmon resonances in spatially dispersive nano-objects: phenomenological treatise," ACS Nano, vol. 7, no. 5, pp. 4334-4342, 2013.

[101] Y. Kosmann-Schwarzbach, Invariance and Conservation Laws in the Twentieth Century, Berlin, Springer-Verlag, 2011.

[102] M. Fox, "Optical Properties of Solids," in ser. Oxford Master Series in Condensed Matter Physics, Oxford, Oxford University Press, 2001.

[103] E. N. Economou, "Surface plasmons in thin films," Phys. Rev., vol. 182, pp. 539-554, 1969.

[104] S. Raza, T. Christensen, M. Wubs, S. I. Bozhevolnyi, and N. A. Mortensen, "Nonlocal response in thin-film waveguides: loss versus nonlocality and breaking of complementarity," Phys. Rev. B, vol. 88, p. 115401, 2013.

[105] K. V. Nerkararyan, "Superfocusing of a surface polariton in a wedge-like structure," Phys. Lett. A, vol. 237, no. 1, pp. 103-105, 1997.

[106] M. I. Stockman, "Nanofocusing of optical energy in tapered plasmonic waveguides," Phys. Rev. Lett., vol. 93, p. 137404 , 2004.

[107] D. K. Gramotnev and S. I. Bozhevolnyi, "Plasmonics beyond the diffraction limit," Nat. Photonics, vol. 4, no. 2, pp. 83-91, 2010.

[108] D. K. Gramotnev and S. I. Bozhevolnyi, "Nanofocusing of electromagnetic radiation,” Nat. Photonics, vol. 8, no. 1, pp. 14-23, 2014.

[109] J. J. Baumberg, J. Aizpurua, M. H. Mikkelsen, and D. R. Smith, "Extreme nanophotonics from ultrathin metallic gaps," Nat. Mater., vol. 18, pp. 668-678, 2019.

[110] Y. Luo, J. B. Pendry, and A. Aubry, "Surface plasmons and singularities," Nano Lett., vol. 10, no. 10, pp. 4186-4191, 2010.

[111] N. A. Mortensen and S. Xiao, "Slow-light enhancement of Beer-Lambert-Bouguer absorption," Appl. Phys. Lett., vol. 90, no. 14, p. 141108, 2007. 
[112] J. G. Pedersen, S. Xiao, and N. A. Mortensen, "Limits of slow light in photonic crystals," Phys. Rev. B, vol. 78, p. 153101, 2008.

[113] J. Grgić, J. R. Ott, F. Wang, et al., "Fundamental limitations to gain enhancement in periodic media and waveguides," Phys. Rev. Lett., vol. 108, p. 183903, 2012.

[114] C. Wolff, K. Busch, and N. A. Mortensen, "Modal expansions in periodic photonic systems with material loss and dispersion," Phys. Rev. B, vol. 97, p. 104203, 2018.

[115] R. W. Alexander, G. S. Kovener, and R. J. Bell, "Dispersion curves for surface electromagnetic waves with damping," Phys. Rev. Lett., vol. 32, pp. 154-157, 1974.

[116] J. B. Khurgin, "How to deal with the loss in plasmonics and metamaterials," Nat. Nanotechnol., vol. 10, p. 2, 2015.

[117] W. Yan, M. Wubs, and N. A. Mortensen, "Hyperbolic metamaterials: nonlocal response regularizes broadband supersingularity," Phys. Rev. B, vol. 86, p. 205429, 2012.

[118] G. Mie, "Beiträge zur Optik trüber Medien, speziell kolloidaler Metallösungen,” Ann. Phys., vol. 330, no. 3, pp. 377-445, 1908.

[119] C. F. Bohren and D. R. Huffman, Absorption and Scattering of Light by Small Particles, New York, Wiley \& Sons, 1998.

[120] T. Christensen, W. Yan, S. Raza, A.-P. Jauho, N. A. Mortensen, and $M$. Wubs, "Nonlocal response of metallic nanospheres probed by light, electrons, and atoms," ACS Nano, vol. 8, no. 2, pp. 1745-1758, 2014.

[121] F. Wang and Y. R. Shen, "General properties of local plasmons in metal nanostructures," Phys. Rev. Lett., vol. 97, p. 206806, 2006.

[122] T. Christensen, W. Yan, A.-P. Jauho, M. Soljačić, and N. A. Mortensen, "Quantum corrections in nanoplasmonics: shape, scale, and material," Phys. Rev. Lett., vol. 118, p. $157402,2017$.

[123] J. Kern, S. Großmann, N. V. Tarakina, et al., "Atomic-scale confinement of resonant optical fields," Nano Lett., vol. 12, no. 11, pp. 5504-5509, 2012.

[124] S. Raza, S. Kadkhodazadeh, T. Christensen, et al., “Multipole plasmons and their disappearance in few-nanometer silver nanoparticles," Nat. Commun., vol. 6, p. 8788, 2015.

[125] S. Raza, N. Stenger, A. Pors, et al., "Extremely confined gap surface-plasmon modes excited by electrons," Nat. Commun., vol. 5, p. 4125, 2014.

[126] H. Duan, A. I. Fernández-Domínguez, M. Bosman, S. A. Maier, and J. K. W. Yang, "Nanoplasmonics: classical down to the nanometer scale," Nano Lett., vol. 12, no. 3, pp. 1683-1689, 2012.

[127] H. Shen, L. Chen, L. Ferrari, et al., "Optical observation of plasmonic nonlocal effects in a 2D superlattice of ultrasmall gold nanoparticles," Nano Lett., vol. 17, no. 4, pp. 2234-2239, 2017.

[128] C. L. C. Smith, N. Stenger, A. Kristensen, N. A. Mortensen, and S. I. Bozhevolnyi, "Gap and channeled plasmons in tapered grooves: a review," Nanoscale, vol. 7, pp. 9355-9386, 2015.

[129] S. Thongrattanasiri and F. J. Garcia de Abajo, "Optical field enhancement by strong plasmon interaction in graphene nanostructures," Phys. Rev. Lett., vol. 110, p. 187401, 2013.
[130] S. Kim, J. Jin, Y.-J. Kim, I.-Y. Park, Y. Kim, and S.-W. Kim, "High-harmonic generation by resonant plasmon field enhancement," Nature, vol. 453, no. 7196, pp. 757-760, 2008.

[131] A. Kinkhabwala, Z. Yu, S. Fan, Y. Avlasevich, K. Müllen, and W. E. Moerner, "Large single-molecule fluorescence enhancements produced by a bowtie nanoantenna," Nat. Photonics, vol. 3, no. 11, pp. 654-657, 2009.

[132] G. P. Acuna, F. M. Möller, P. Holzmeister, S. Beater, B. Lalkens, and P. Tinnefeld, "Fluorescence enhancement at docking sites of dna-directed self-assembled nanoantennas," Science, vol. 338, no. 6106, pp. 506-510, 2012.

[133] R. Jin, “Nanoparticle clusters light up in SERS," Angew. Chem. Int. Ed., vol. 49, no. 16, pp. 2826-2829, 2010.

[134] C. Ciracì, R. T. Hill, J. J. Mock, et al., "Probing the ultimate limits of plasmonic enhancement," Science, vol. 337 , no. 6098, pp. 1072-1074, 2012.

[135] E. Prodan, C. Radloff, N. J. Halas, and P. Nordlander, “A hybridization model for the plasmon response of complex nanostructures," Science, vol. 302, no. 5644, pp. 419-422, 2003.

[136] F. Ouyang, P. E. Batson, and M. Isaacson, "Quantum size effects in the surface-plasmon excitation of small metallic particles by electron-energy-loss spectroscopy," Phys. Rev. $B$, vol. 46, pp. 15421-15425, 1992.

[137] J. Tiggesbäumker, L. Köller, K.-H. Meiwes-Broer, and A. Liebsch, "Blue shift of the Mie plasma frequency in $\mathrm{Ag}$ clusters and particles," Phys. Rev. A, vol. 48, pp. R1749-R1752, 1993.

[138] S. Raza, N. Stenger, S. Kadkhodazadeh, et al., "Blueshift of the surface plasmon resonance in silver nanoparticles studied with EELS," Nanophotonics, vol. 2, no. 2, pp. 131-138, 2013.

[139] J. A. Scholl, A. L. Koh, and J. A. Dionne, "Quantum plasmon resonances of individual metallic nanoparticles," Nature, vol. 483, no. 7390, p. 421, 2012.

[140] M. Hu, C. Novo, A. Funston, et al., "Dark-field microscopy studies of single metal nanoparticles: understanding the factors that influence the linewidth of the localized surface plasmon resonance," J. Mater. Chem., vol. 18, pp. 1949-1960, 2008.

[141] S. Raza, W. Yan, N. Stenger, M. Wubs, and N. A. Mortensen, "Blueshift of the surface plasmon resonance in silver nanoparticles: substrate effects," Opt. Express, vol. 21, no. 22, pp. 27344-27355, 2013.

[142] F. J. García de Abajo, "Plasmons go quantum," Nature, vol. 483, pp. 417-418, 2012.

[143] H. Haberland, "Looking from both sides," Nature, vol. 494 , pp. E1-E2, 2013.

[144] W. P. Halperin, "Quantum size effects in metal particles," Rev. Mod. Phys., vol. 58, pp. 533-606, 1986.

[145] K. P. Charlé, W. Schulze, and B. Winter, "The size dependent shift of the surface-plasmon absorption-band of small spherical metal particles," Z. Physik D Atoms, Mol. Clust., vol. 12, nos 1-4, pp. 471-475, 1989.

[146] U. Kreibig and L. Genzel, "Optical absorption of small metallic particles,” Surf. Sci., vol. 156, pp. 678-700, 1985. 
[147] L. Genzel, T. P. Martin, and U. Kreibig, "Dielectric function and plasma resonance of small metal particles," $Z$. Phys. $B$, vol. 21, pp. 339-346, 1975.

[148] U. Kreibig and M. Vollmer, Optical Properties of Metal Clusters, Berlin, Springer-Verlag, 1995.

[149] C. Tserkezis, J. R. Maack, Z. Liu, M. Wubs, and N. A. Mortensen, "Robustness of the far-field response of nonlocal plasmonic ensembles," Sci. Rep., vol. 6, p. 28441, 2016.

[150] P. Apell and D. R. Penn, "Optical properties of small metal spheres: surface effects," Phys. Rev. Lett., vol. 50, pp. 1316-1319, 1983.

[151] W. A. Kraus and G. C. Schatz, "Plasmon resonance broadening in small metal particles," J. Chem. Phys., vol. 79, no. 12 , pp. $6130-6139,1983$.

[152] Å. Ljungbert and P. Apell, "The role of electron-hole pair excitations in the optical absorption of metals, particularly metal spheres," Solid State Commun., vol. 46, no. 1, pp. 47-50, 1983.

[153] A. Ljungbert and S. Lundqvist, "Non-local effects in the optical absorption of small metallic particles," Surf. Sci., vol. 156, pp. 839-844, 1985.

[154] A. V. Uskov, I. E. Protsenko, N. A. Mortensen, and E. P. O'Reilly, "Broadening of plasmonic resonances due to electron collisions with nanoparticle boundary: quantum-mechanical consideration," Plasmonics, vol. 9, pp. 185-192, 2014.

[155] L. D. Landau, "On the vibrations of the electronic plasma," Zh. Eksp. Teor. Fiz., vol. 10, p. 25, 1946.

[156] J. Khurgin, W.-Y. Tsai, D. P. Tsai, and G. Sun, “Landau damping and limit to field confinement and enhancement in plasmonic dimers," ACS Photonics, vol. 4, no. 11, pp. 2871-2880, 2017.

[157] J. B. Khurgin and G. Sun, "Landau damping - the ultimate limit of field confinement and enhancement in plasmonic structures," in Springer Series in Solid-State Sciences, vol. 185, Berlin, Springer International Publishing, 2017, pp. 303-322.

[158] N. A. Mortensen, J. B. Khurgin, and M. Wubs, "Nonlocality in plasmonics," in World Scientific Handbook of Metamaterials and Plasmonics, vol. 4, Singapore, World Scientific, 2017, pp. 67-113.

[159] K. J. Savage, M. M. Hawkeye, R. Esteban, A. G. Borisov, J. Aizpurua, and J. J. Baumberg, "Revealing the quantum regime in tunnelling plasmonics," Nature, vol. 491, no. 7425, pp. 574-577, 2012.

[160] S. Kadkhodazadeh, J. B. Wagner, H. Kneipp, and K. Kneipp, "Coexistence of classical and quantum plasmonics in large plasmonic structures with subnanometer gaps," Appl. Phys. Lett., vol. 103, no. 8, p. 083103, 2013.

[161] S. F. Tan, L. Wu, J. K. W. Yang, P. Bai, M. Bosman, and C. A. Nijhuis, "Quantum plasmon resonances controlled by molecular tunnel junctions," Science, vol. 343, no. 6178, pp. 1496-1499, 2014.

[162] H. Cha, J. H. Yoon, and S. Yoon, "Probing quantum plasmon coupling using gold nanoparticle dimers with tunable interparticle distances down to the subnanometer range," ACS Nano, vol. 8, no. 8, pp. 8554-8563, 2014.
[163] J.-H. Song, S. Raza, J. van de Groep, et al., "Nanoelectromechanical modulation of a stronglycoupled plasmonic dimer," Nat. Commun., vol. 12, p. 48, 2021.

[164] R. Esteban, A. G. Borisov, P. Nordlander, and J. Aizpurua, "Bridging quantum and classical plasmonics with a quantum-corrected model," Nat. Commun., vol. 3, no. 10, p. 825, 2012.

[165] N. A. Mortensen, "Nonlocal formalism for nanoplasmonics: phenomenological and semi-classical considerations," Photonics Nanostruct., vol. 11, no. 4, pp. 303-308, 2013.

[166] B. Gallinet, J. Butet, and O. J. F. Martin, "Numerical methods for nanophotonics: standard problems and future challenges," Laser Photon. Rev., vol. 9, no. 6, pp. 577-603, 2015.

[167] R. W. Schoonover, J. M. Rutherford, O. Keller, and P. S. Carney, "Non-local constitutive relations and the quasi-homogeneous approximation," Phys. Lett. A, vol. 342 , no. 5, pp. 363-367, 2005.

[168] N. A. Mortensen, S. Raza, M. Wubs, T. Søndergaard, and S. I. Bozhevolnyi, "A generalized nonlocal optical response theory for plasmonic nanostructures," Nat. Commun., vol. 5 , p. 3809, 2014.

[169] N. A. Mortensen, G. Toscano, S. Raza, et al., “Nanoplasmonics beyond Ohm's law," AIP Conf. Proc., vol. 1475 , no. 1, pp. $28-32,2012$.

[170] H. Wallén, H. Kettunen, and A. Sihvola, "Surface modes of negative-parameter interfaces and the importance of rounding sharp corners," Metamaterials, vol. 2, no. 2, pp. 113-121, 2008.

[171] L. C. Davis, "Electrostatic edge modes of a dielectric wedge," Phys. Rev. B, no. 14, pp. 5523-5525, 1976. https://doi.org/10 $.1103 /$ physrevb.14.5523.

[172] J. J. Monaghan, "Smoothed particle hydrodynamics," Rep. Prog. Phys., vol. 68, no. 8, pp. 1703-1759, 2005.

[173] G. Toscano, S. Raza, A.-P. Jauho, N. A. Mortensen, and M. Wubs, "Modified field enhancement and extinction in plasmonic nanowire dimers due to nonlocal response," Opt. Express, vol. 20, no. 4, pp. 4176-4188, 2012.

[174] W. Yan and N. A. Mortensen, "Nonclassical effects in plasmonics: an energy perspective to quantify nonclassical effects," Phys. Rev. B, vol. 93, p. 115439, 2016.

[175] M. Fleischmann, P. J. Hendra, and A. J. McQuillan, "Raman spectra of pyridine adsorbed at a silver electrode," Chem. Phys. Lett., vol. 262, pp. 163-166, 1974.

[176] M. Moskovits, "Surface-enhanced spectroscopy," Rev. Mod. Phys., vol. 57, pp. 783-826, 1985.

[177] K. Kneipp, "Surface-enhanced Raman scattering," Phys. Today, vol. 60, no. 11, pp. 40-46, 2007.

[178] J. Langer, D. Jimenez de Aberasturi, J. Aizpurua, et al., "Present and future of surface-enhanced Raman scattering," ACS Nano, vol. 14, no. 1, pp. 28-117, 2020.

[179] F. J. García-Vidal and J. B. Pendry, "Collective theory for surface enhanced Raman scattering," Phys. Rev. Lett., vol. 77, pp. 1163-1166, 1996.

[180] S. Xiao, N. A. Mortensen, and A.-P. Jauho, "Nanostructure design for surface-enhanced Raman spectroscopy - prospects and limits," J. Eur. Opt. Soc.-Rapid Publ., vol. 3, p. 08022, 2008. 
[181] G. Toscano, S. Raza, S. Xiao, et al., "Surface-enhanced Raman spectroscopy: nonlocal limitations," Opt. Lett., vol. 37, no. 13, pp. 2538-2540, 2012.

[182] A. D. Boardman, Electromagnetic Surface Modes, New York, Wiley \& Sons, 1982.

[183] S. Raza, S. I. Bozhevolnyi, M. Wubs, and N. A. Mortensen, "Nonlocal optical response in metallic nanostructures," J. Phys.: Condens. Matter, vol. 27, p. 183204, 2015.

[184] C. Ciracì, E. Poutrina, M. Scalora, and D. R. Smith, "Second-harmonic generation in metallic nanoparticles: clarification of the role of the surface," Phys. $\operatorname{Rev}$. B, vol. 86, p. 115451, 2012.

[185] M. Moeferdt, T. Kiel, T. Sproll, F. Intravaia, and K. Busch, "Plasmonic modes in nanowire dimers: a study based on the hydrodynamic Drude model including nonlocal and nonlinear effects," Phys. Rev. B, vol. 97, p. 075431, 2018.

[186] A. V. Krasavin, P. Ginzburg, and A. V. Zayats, “Free-electron optical nonlinearities in plasmonic nanostructures: a review of the hydrodynamic description," Laser Photon. Rev., vol. 12, no. 1, p. 1700082, 2018.

[187] N. D. Lang and W. Kohn, "Theory of metal surfaces: charge density and surface energy," Phys. Rev. B, vol. 1, pp. 4555-4568, 1970.

[188] J. Friedel, "The distribution of electrons round impurities in monovalent metals," Philos. Mag. A, vol. 43, no. 337, pp. 153-189, 1952.

[189] S. Raza, G. Toscano, A.-P. Jauho, M. Wubs, and N. A. Mortensen, "Unusual resonances in nanoplasmonic structures due to nonlocal response," Phys. Rev. B, vol. 84, p. 121412(R), 2011.

[190] P. Halevi, "Hydrodynamic model for the degenerate free-electron gas: generalization to arbitrary frequencies," Phys. Rev. B, vol. 51, pp. 7497-7499, 1995.

[191] S. I. Pekar, "Theory of electromagnetic waves in a crystal with excitons," J. Phys. Chem. Solid., vol. 5, nos 1-2, pp. 11-22, 1958.

[192] P. Halevi and G. Hernández-Cocoletzi, “Additional boundary conditions: critical comparison between theory and experiment," Phys. Rev. Lett., vol. 48, pp. 1500-1503, 1982.

[193] K. Henneberger, “Additional boundary conditions: an historical mistake," Phys. Rev. Lett., vol. 80, pp. 2889-2892, 1998.

[194] G. Toscano, S. Raza, W. Yan, et al., "Nonlocal response in plasmonic waveguiding with extreme light confinement," Nanophotonics, vol. 2, no. 3, pp. 161-166, 2013.

[195] R. H. Ritchie and A. L. Marusak, "The surface plasmon dispersion relation for an electron gas," Surf. Sci., vol. 4, no. 3, pp. $234-240,1966$.

[196] R. Fuchs and K. L. Kliewer, "Optical properties of an electron gas: further studies of a nonlocal description," Phys. Rev., vol. 185, pp. 905-913, 1969.

[197] P. Jewsbury, "Electrodynamic boundary conditions at metal interfaces,"J. Phys. F Met. Phys., vol. 11, p. 195, 1981.

[198] M. A. Day, "The no-slip condition of fluid dynamics," Erkenntnis, vol. 33, no. 3, pp. 285-296, 1990.

[199] A. Liebsch, "Surface-plasmon dispersion and size dependence of Mie resonance: silver versus simple metals,"
Phys. Rev. B, vol. 48, no. 15, pp. 11317-11328,

1993.

[200] R. C. Monreal, T. J. Antosiewicz, and S. P. Apell, "Competition between surface screening and size quantization for surface plasmons in nanoparticles," New J. Phys., vol. 15, no. 8, p. 083044, 2013.

[201] T. V. Teperik, P. Nordlander, J. Aizpurua, and A. G. Borisov, "Robust subnanometric plasmon ruler by rescaling of the nonlocal optical response,” Phys. Rev. Lett., vol. 110, p. 263901, 2013.

[202] W. Yan, M. Wubs, and N. A. Mortensen, "Projected dipole model for quantum plasmonics," Phys. Rev. Lett., vol. 115, p. 137403, 2015.

[203] O. Keller, M. Xiao, and S. I. Bozhevolnyi, “Optical diamagnetic polarizability of a mesoscopic metallic sphere: transverse self-field approach," Opt. Commun., vol. 102, no. 3, pp. 238-244, 1993.

[204] M. Ichikawa, "Theory of localized plasmons for metal nanostructures in random-phase approximation," J. Phys. Soc. Jpn, vol. 80, no. 4, p. 044606, 2011.

[205] Z. F. Öztürk, S. Xiao, M. Yan, M. Wubs, A.-P. Jauho, and N. A. Mortensen, "Field enhancement at metallic interfaces due to quantum confinement,"J. Nanophotonics, vol. 5, no. 1, p. 051602, 2011.

[206] C. David and F. J. García de Abajo, "Surface plasmon dependence on the electron density profile at metal surfaces," ACS Nano, vol. 8, no. 9, pp. 9558-9566, 2014.

[207] G. Toscano, J. Straubel, A. Kwiatkowski, et al., "Resonance shifts and spill-out effects in self-consistent hydrodynamic nanoplasmonics," Nat. Commun., vol. 6, p. 7132, 2015.

[208] W. Yan, "Hydrodynamic theory for quantum plasmonics: linear-response dynamics of the inhomogeneous electron gas," Phys. Rev. B, vol. 91, p. 115416, 2015.

[209] C. Ciracì and F. Della Sala, "Quantum hydrodynamic theory for plasmonics: impact of the electron density tail," Phys. Rev. B, vol. 93, p. 205405, 2016.

[210] M. Wubs, "Classification of scalar and dyadic nonlocal optical response models," Opt. Express, vol. 23, no. 24, pp. 31296-31312, 2015.

[211] A. R. Melnyk and M. J. Harrison, "Resonant excitation of plasmons in thin films by electromagnetic waves," Phys. Rev. Lett., vol. 21, pp. 85-88, 1968.

[212] I. Lindau and P. O. Nilsson, "Experimental evidence for excitation of longitudinal plasmons by photons," Phys. Lett. A, vol. 31, no. 7, pp. 352-353, 1970.

[213] M. Anderegg, B. Feuerbacher, and B. Fitton, "Optically excited longitudinal plasmons in potassium," Phys. Rev. Lett., vol. 27, pp. 1565-1568, 1971.

[214] I. Romero, J. Aizpurua, G. W. Bryant, and F. J. García de Abajo, "Plasmons in nearly touching metallic nanoparticles: singular response in the limit of touching dimers," Opt. Express, vol. 14, no. 21, pp. 9988-9999, 2006.

[215] A. Aubry, D. Y. Lei, S. A. Maier, and J. B. Pendry, "Interaction between plasmonic nanoparticles revisited with transformation optics," Phys. Rev. Lett., vol. 105, p. 233901, 2010.

[216] C. David and F. J. García de Abajo, "Spatial nonlocality in the optical response of metal nanoparticles," J. Phys. Chem. C, vol. 115, no. 40, pp. 19470-19475, 2011. 
[217] A. I. Fernández-Domínguez, A. Wiener, F. J. García-Vidal, S. A. Maier, and J. B. Pendry, "Transformation-optics description of nonlocal effects in plasmonic nanostructures," Phys. Rev. Lett., vol. 108, p. 106802, 2012.

[218] A. I. Fernández-Domínguez, P. Zhang, Y. Luo, S. A. Maier, F. J. García-Vidal, and J. B. Pendry, "Transformation-optics insight into nonlocal effects in separated nanowires," Phys. Rev. B, vol. 86, p. 241110, 2012.

[219] C. Ciracì, Y. Urzhumov, and D. R. Smith, "Effects of classical nonlocality on the optical response of three-dimensional plasmonic nanodimers," J. Opt. Soc. Am. B, vol. 30, no. 10, pp. 2731-2736, 2013.

[220] S. Raza, M. Wubs, S. I. Bozhevolnyi, and N. A. Mortensen, "Nonlocal study of ultimate plasmon hybridization," Opt. Lett., vol. 40, no. 5, pp. 839-842, 2015.

[221] L. D. Landau, E. M. Lifshitz, and L. P. Pitaevskii, “Course on theoretical physics," in Electrodynamics of Continuous Media, Oxford, Butterworth Heinemann, 1984.

[222] K. Seeger, "Semiconductor physics," in Springer Series in Solid-State Sciences, Berlin, Springer, 1991.

[223] N. A. Mortensen, L. H. Olesen, L. Belmon, and H. Bruus, "Electrohydrodynamics of binary electrolytes driven by modulated surface potentials," Phys. Rev. E, vol. 71, p. 056306, 2005.

[224] K. R. Hiremath, L. Zschiedrich, and F. Schmidt, "Numerical solution of nonlocal hydrodynamic Drude model for arbitrary shaped nano-plasmonic structures using Nédélec finite elements," J. Comput. Phys., vol. 231, no. 17, pp. 5890-5896, 2012.

[225] L. Li, S. Lanteri, N. A. Mortensen, and M. Wubs, “A hybridizable discontinuous Galerkin method for solving nonlocal optical response models," Comput. Phys. Commun., vol. 219, pp. 99-107, 2017.

[226] W. Yan, N. A. Mortensen, and M. Wubs, “Green's function surface-integral method for nonlocal response of plasmonic nanowires in arbitrary dielectric environments," Phys. Rev. B, vol. 88, p. 155414, 2013.

[227] A. Yanai, N. A. Mortensen, and U. Levy, "Absorption and eigenmode calculation for one-dimensional periodic metallic structures using the hydrodynamic approximation," Phys. Rev. B, vol. 88, p. 205120, 2013.

[228] D. Chulhai and L. Jensen, "Generalized nonlocal optical response," in nanoHUB, 2016.

[229] E. H. Sondheimer, "The mean free path of electrons in metals," Adv. Phys., vol. 1, no. 1, pp.1-42, 1952.

[230] G. Manfredi, "Preface to special topic: plasmonics and solid state plasmas," Phys. Plasmas, vol. 25, no. 3, p. 031701, 2018.

[231] Z. A. Moldabekov, M. Bonitz, and T. S. Ramazanov, "Theoretical foundations of quantum hydrodynamics for plasmas,” Phys. Plasmas, vol. 25, no. 3, p. 031903, 2018.

[232] X. Li, H. Fang, X. Weng, et al., "Electronic spill-out induced spectral broadening in quantum hydrodynamic nanoplasmonics," Opt. Express, vol. 23, no. 23 , pp. 29738-29745, 2015.

[233] X. Li, D. Xiao, and Z. Zhang, "Landau damping of quantum plasmons in metal nanostructures," New J. Phys., vol. 15, p. $023011,2013$.
[234] J. Zuloaga, E. Prodan, and P. Nordlander, "Quantum description of the plasmon resonances of a nanoparticle dimer," Nano Lett., vol. 9, pp. 887-891, 2009.

[235] J. Zuloaga, E. Prodan, and P. Nordlander, "Quantum plasmonics: optical properties and tunability of metallic nanorods," ACS Nano, vol. 4, no. 9, pp. 5269-5276, 2010.

[236] T. V. Teperik, P. Nordlander, J. Aizpurua, and A. G. Borisov, "Quantum effects and nonlocality in strongly coupled plasmonic nanowire dimers," Opt. Express, vol. 21, no. 22, pp. 27306-27325, 2013.

[237] L. Stella, P. Zhang, F. J. García-Vidal, A. Rubio, and P. García-González, "Performance of nonlocal optics when applied to plasmonic nanostructures," J. Phys. Chem. C, vol. 117, no. 17, pp. 8941-8949, 2013.

[238] K. Andersen, K. L. Jensen, N. A. Mortensen, and K. S. Thygesen, "Visualizing hybridized quantum plasmons in coupled nanowires: from classical to tunneling regime," Phys. Rev. B, no. 87, p. 235433, 2013. https://doi.org/10 $.1103 /$ physrevb.87.235433.

[239] M. G. Blaber, M. D. Arnold, and M. J. Ford, "Search for the ideal plasmonic nanoshell: the effects of surface scattering and alternatives to gold and silver," J. Phys. Chem. C, vol. 113, no. 8, pp. 3041-3045, 2009.

[240] J. Rammer and H. Smith, "Quantum field-theoretical methods in transport theory of metals," Rev. Mod. Phys., vol. 58, pp. 323-359, 1986.

[241] I. Tokatly and O. Pankratov, "Hydrodynamic theory of an electron gas," Phys. Rev. B, vol. 60, pp. 15550-15553, 1999.

[242] M. K. Svendsen, C. Wolff, A.-P. Jauho, N. A. Mortensen, and C. Tserkezis, "Role of diffusive surface scattering in nonlocal plasmonics," J. Phys.: Condens. Matter, vol. 32, no. 39, p. 395702, 2020

[243] J. B. Khurgin, "Ultimate limit of field confinement by surface plasmon polaritons," Faraday Discuss., vol. 178, pp. 109-122, 2015.

[244] C. Tserkezis, W. Yan, W. Hsieh, et al., "On the origin of nonlocal damping in plasmonic monomers and dimers," Int. J. Mod. Phys. B, vol. 31, no. 24, p. 1740005, 2017.

[245] U. Kreibig and C. von Fragstein, "The limitation of electron mean free path in small silver particles," Z. Phys., vol. 224, no. 4, pp. 307-323, 1969.

[246] K.-P. Charlé, F. Frank, and W. Schulze, “The optical properties of silver microcrystallites in dependence on size and the influence of the matrix environment," Ber. Bunsenges. Phys. Chem., vol. 88, no. 4, pp. 350-354, 1984.

[247] M. Gaudry, E. Cottancin, M. Pellarin, et al., "Size and composition dependence in the optical properties of mixed (transition metal/noble metal) embedded clusters," Phys. Rev. B, vol. 67, p. 155409, 2003.

[248] S. Berciaud, L. Cognet, P. Tamarat, and B. Lounis, "Observation of intrinsic size effects in the optical response of individual gold nanoparticles," Nano Lett., vol. 5, no. 3 , pp. 515-518, 2005.

[249] L. B. Scaffardi and J. O. Tocho, "Size dependence of refractive index of gold nanoparticles," Nanotechnology, vol. 17, no. 5, pp. 1309-1315, 2006

[250] K. Kolwas and A. Derkachova, "Damping rates of surface plasmons for particles of size from nano- to micrometers; 
reduction of the nonradiative decay," J. Quant. Spectrosc.

Radiat. Transfer, vol. 114, pp. 45-55, 2013.

[251] A. Wiener, H. Duan, M. Bosman, et al., "Electron-energy loss study of nonlocal effects in connected plasmonic nanoprisms," ACS Nano, vol. 7, no. 7, pp. 6287-6296, 2013.

[252] R. Esteban, A. Zugarramurdi, P. Zhang, et al., "A classical treatment of optical tunneling in plasmonic gaps: extending the quantum corrected model to practical situations," Faraday Discuss., vol. 178, pp. 151-183, 2015.

[253] A. Varas, P. García-González, J. Feist, F. J. García-Vidal, and A. Rubio, "Quantum plasmonics: from jellium models to ab initio calculations," Nanophotonics, vol. 5, pp. 409-426, 2016.

[254] J. A. Scholl, A. Garcia-Etxarri, A. L. Koh, and J. A. Dionne, "Observation of quantum tunneling between two plasmonic nanoparticles," Nano Lett., vol. 13, no. 2, pp. 564-569, 2013.

[255] J. Mertens, A. L. Eiden, D. O. Sigle, et al., "Controlling subnanometer gaps in plasmonic dimers using graphene," Nano Lett., vol. 13, no. 11, pp. 5033-5038, 2013.

[256] H. Jung, H. Cha, D. Lee, and S. Yoon, "Bridging the nanogap with light: continuous tuning of plasmon coupling between gold nanoparticles," ACS Nano, vol. 9, no. 12, pp. 12292-12300, 2015.

[257] A. Bagchi, R. G. Barrera, and A. K. Rajagopal, "Perturbative approach to the calculation of the electric field near a metal surface," Phys. Rev. B, vol. 20, pp. 4824-4838, 1979.

[258] P. J. Feibelman, "Interpretation of differential reflectance studies of metal surfaces," Phys. Rev. B, vol. 23, pp. 2629-2634, 1981.

[259] P. Apell, "On the surface photoelectric effect in aluminum," Phys. Scripta, vol. 25, no. 1A, pp. 57-64, 1982.

[260] A. Liebsch, Electronic Excitations at Metal Surfaces, New York, Springer, 1997.

[261] H.-Y. Deng, "A theory of electrodynamic response for bounded metals: surface capacitive effects," Ann. Phys., vol. 418, p. 168204, 2020.

[262] J. E. Sipe, "Bulk-selvedge coupling theory for the optical properties of surfaces," Phys. Rev. B, vol. 22, pp. 1589-1599, 1980.

[263] Y. Luo, A. I. Fernandez-Dominguez, A. Wiener, S. A. Maier, and J. B. Pendry, "Surface plasmons and nonlocality: a simple model," Phys. Rev. Lett., vol. 111, p. 093901, 2013.

[264] D. Jin, Q. Hu, D. Neuhauser, et al., "Quantum-spilloverenhanced surface-plasmonic absorption at the interface of silver and high-index dielectrics," Phys. Rev. Lett., vol. 115, p. 193901, 2015.

[265] A. Campos, N. Troc, E. Cottancin, et al., "Plasmonic quantum size effects in silver nanoparticles are dominated by interfaces and local environments," Nat. Phys., vol. 15, no. 3, pp. 275-280, 2019.

[266] P. Apell, "A simple derivation of the surface contribution to the reflectivity of a metal, and its use in the van der Waals interaction," Phys. Scripta, vol. 24, no. 4, pp. 795-806, 1981.

[267] F. Forstmann and R. R. Gerhardts, Metal Optics Near the Plasma Frequency, Berlin Heidelberg, Springer-Verlag, 1986.
[268] D. C. Langreth, "Macroscopic approach to the theory of reflectivity," Phys. Rev. B, vol. 39, pp.10020-10027, 1989.

[269] Y. Yang, D. Zhu, W. Yan, et al., "A general theoretical and experimental framework for nanoscale electromagnetism," Nature, vol. 576, pp. 248-252, 2019.

[270] R. Sinha-Roy, P. García-González, H.-C. Weissker, F. Rabilloud, and A. I. Fernández-Domínguez, "Classical and ab initio plasmonics meet at sub-nanometric noble metal rods," ACS Photonics, vol. 4, no. 6, pp. 1484-1493, 2017.

[271] N. A. Mortensen, P. A. D. Gonçalves, F. A. Shuklin, et al., "Surface-response functions obtained from equilibrium electron-density profiles," Nanophotonics, vol. 10, 2021. https://doi.org/10.1515/nanoph-2021-0084.

[272] P. J. Feibelman, "Microscopic calculation of electromagnetic fields in refraction at a jellium-vacuum interface," Phys. Rev. $B$, vol. 12, pp. 1319-1336, 1975.

[273] P. Ahlqvist and P. Apell, "On the hydrodynamical theory for surface plasmons," Phys. Scripta, vol. 25, no. 4, pp. 587-591, 1982.

[274] O. Keller, "Random-phase-approximation study of the response function describing optical second-harmonic generation from a metal selvedge," Phys. Rev. B, vol. 33, pp. 990-1009, 1986.

[275] M. Ichikawa, "Theory of localized plasmons for metal nanostructures in dielectrics," e-J. Surf. Sci. Nanotech., vol. 16, pp. 329-338, 2018.

[276] A. Bagchi, N. Kar, and R. G. Barrera, "Effect of refraction of $p$-polarized light on angle-resolved photoemission from surface states on metals," Phys. Rev. Lett., vol. 40, pp. 803-806, 1978.

[277] X. Liu, H. Kang, H. Yuan, et al., "Electrical tuning of a quantum plasmonic resonance," Nat. Nanotechnol., vol. 12, no. 9 , pp. 866-870, 2017.

[278] E. J. H. Skjølstrup, T. Søndergaard, and T. G. Pedersen, "Quantum spill-out in few-nanometer metal gaps: effect on gap plasmons and reflectance from ultrasharp groove arrays," Phys. Rev. B, vol. 97, p. 115429, 2018.

[279] E. J. H. Skjølstrup, T. Søndergaard, and T. G. Pedersen, "Quantum spill-out in nanometer-thin gold slabs: effect on the plasmon mode index and the plasmonic absorption," Phys. Rev. B, vol. 99, p. 155427, 2019.

[280] A. Taghizadeh and T. G. Pedersen, "Plasmons in ultra-thin gold slabs with quantum spill-out: Fourier modal method, perturbative approach, and analytical model," Opt. Express, vol. 27, no. 25, pp. 36941-36952, 2019.

[281] A. Rivacoba, "Electron spill-out effects in plasmon excitations by fast electrons," Ultramicroscopy, vol. 207, p. 112835, 2019.

[282] C. Henkel, G. Boedecker, and M. Wilkens, "Local fields in a soft matter bubble,” Appl. Phys. B, vol. 93, pp. 217-221, 2008.

[283] P. Apell, "Effects of non-locality and surface diffuseness on the electromagnetic response of a vacuum metal interface," Solid State Commun., vol. 47, no. 8 , pp. 619-622, 1983.

[284] A. Liebsch, "Dynamical screening at simple-metal surfaces," Phys. Rev. B, vol. 36, pp. 7378-7388, 1987. 
[285] J. M. Rogowska, K. F. Wojciechowski, and M. Maciejewski, "Analytical representation of the Lang-Kohn density profiles by the numerical fitting," Acta Phys. Pol., A, vol. 85, pp. 593-601, 1994.

[286] M. Hannemann, G. Wegner, and C. Henkel, "No-slip boundary conditions for electron hydrodynamics and the thermal Casimir pressure," Universe, vol. 7, no. 4, p. 108, 2021.

[287] F. Cheng, C.-J. Lee, J. Choi, et al., "Epitaxial growth of optically thick, single crystalline silver films for plasmonics," ACS Appl. Mater. Interfaces, vol. 11, no. 3, pp. 3189-3195, 2019.

[288] M. Barbry, P. Koval, F. Marchesin, et al., "Atomistic near-field nanoplasmonics: reaching atomic-scale resolution in nanooptics," Nano Lett., vol. 15, no. 5, pp. 3410-3419, 2015.

[289] P. Zhang, J. Feist, A. Rubio, P. García-González, and F. J. García-Vidal, "Ab initio nanoplasmonics: the impact of atomic structure," Phys. Rev. B, vol. 90, p. 161407(R), 2014.

[290] E. V. Chulkov, V. M. Silkin, and P. M. Echenique, "Image potential states on metal surfaces: binding energies and wave functions," Surf. Sci., vol. 437, no. 3, pp. 330-352, 1999.

[291] A. R. Echarri, J. D. Cox, and F. J. García de Abajo, "Quantum effects in the acoustic plasmons of atomically thin heterostructures," Optica, vol. 6, no. 5, pp. 630-641, 2019.

[292] A. R. Echarri, P. A. D. Gonçalves, C. Tserkezis, F. J. García de Abajo, N. A. Mortensen, and J. D. Cox, “Optical response of noble metal nanostructures: quantum surface effects in crystallographic facets," Optica, vol. 8, no. 5, pp. 710-721, 2021.

[293] M. B. Lundeberg, Y. Gao, R. Asgari, et al., "Tuning quantum nonlocal effects in graphene plasmonics," Science, vol. 357, no. 6347, pp. 187-190, 2017.

[294] E. J. C. Dias, D. A. Iranzo, P. A. D. Gonçalves, et al., "Probing nonlocal effects in metals with graphene plasmons," Phys. Rev. B, vol. 97, p. 245405, 2018.

[295] P. A. D. Gonçalves, T. Christensen, N. M. R. Peres, et al., "Quantum surface-response of metals revealed by acoustic graphene plasmons," Nat. Commun., vol. 12, p. 3271, 2021.

[296] P. A. D. Gonçalves, T. Christensen, N. Rivera, A.-P. Jauho, N. A. Mortensen, and M. Soljačić, "Plasmon-emitter interactions at the nanoscale," Nat. Commun., vol. 11, p. 366, 2020.

[297] P. Apell and $\AA$ Ljungbert, "Red shift of surface plasmons in small metal particles," Solid State Commun., vol. 44, no. 9, pp. 1367-1369, 1982.

[298] J. Lee, D.-J. Jeon, and J.-S. Yeo, "Quantum plasmonics: energy transport through plasmonic gap," Adv. Mater., vol. 33, p. 2006606, 2021.

[299] T. Søndergaard, Green's Function Integral Equation Methods in Nano-Optics, Boca Raton, Florida, CRC Press, 2019.

[300] X. Zheng, M. Kupresak, R. Mittra, and G. A. E. Vandenbosch, "A boundary integral equation scheme for simulating the nonlocal hydrodynamic response of metallic antennas at deep-nanometer scales," IEEE Trans. Antennas Propag., vol. 66, no. 9, pp. 4759-4771, 2018.

[301] X. Zheng, M. Kupresak, N. Verellen, V. V. Moshchalkov, and G. A. E. Vandenbosch, "A review on the application of integral equation-based computational methods to scattering problems in plasmonics," Adv. Theory Simul., vol. 2, no. 9, p. 1900087, 2019.

[302] F. Ouyang and M. Isaacson, "Surface plasmon excitation of objects with arbitrary shape and dielectric constant," Phil. Mag. B, vol. 60, no. 4, pp. 481-492, 1989.

[303] F. J. García de Abajo and J. Aizpurua, "Numerical simulation of electron energy loss near inhomogeneous dielectrics," Phys. Rev. B, vol. 56, pp. 15873-15884, 1997.

[304] R. Yu, L. M. Liz-Marzán, and F. J. García de Abajo, "Universal analytical modeling of plasmonic nanoparticles," Chem. Soc. Rev., vol. 46, pp. 6710-6724, 2017.

[305] A. Raman, W. Shin, and S. Fan, "Upper bound on the modal material loss rate in plasmonic and metamaterial systems," Phys. Rev. Lett., vol. 110, p. 183901, 2013.

[306] P. Apell and Å. Ljungbert, “A general non-local theory for the electromagnetic response of a small metal particle," Phys. Scripta, vol. 26, no. 2, pp. 113-118, 1982.

[307] R. Ruppin, "Optical properties of a plasma sphere," Phys. Rev. Lett., vol. 31, pp. 1434-1437, 1973.

[308] A. D. Boardman and B. V. Paranjape, "Optical surface modes of metal spheres,”J. Phys. FMet. Phys., vol. 7, no. 9, pp. 1935-1945, 1977.

[309] B. B. Dasgupta and R. Fuchs, "Polarizability of a small sphere including nonlocal effects," Phys. Rev. B, vol. 24, pp. 554-561, 1981.

[310] R. Fuchs and F. Claro, "Multipolar response of small metallic spheres: nonlocal theory," Phys. Rev. B, vol. 35, pp. 3722-3727, 1987.

[311] F. J. García de Abajo, "Nonlocal effects in the plasmons of strongly interacting nanoparticles, dimers, and waveguides," J. Phys. Chem. C, vol. 112, no. 46, pp. 17983-17987, 2008.

[312] J. M. McMahon, S. K. Gray, and G. C. Schatz, "Nonlocal optical response of metal nanostructures with arbitrary shape," Phys. Rev. Lett., vol. 103, p. 097403, 2009.

[313] J. M. McMahon, S. K. Gray, and G. C. Schatz, "Calculating nonlocal optical properties of structures with arbitrary shape," Phys. Rev. B, vol. 82, p. 035423, 2010.

[314] J. M. McMahon, S. K. Gray, and G. C. Schatz, "Optical properties of nanowire dimers with a spatially nonlocal dielectric function," Nano Lett., vol. 10, no. 9, pp. 3473-3481, 2010.

[315] J. M. McMahon, S. K. Gray, and G. C. Schatz, "Nonlocal dielectric effects in core-shell nanowires," J. Phys. Chem. C, vol. 114, no. 38, pp. 15903-15908, 2010.

[316] G. Toscano, M. Wubs, S. Xiao, et al., "Plasmonic nanostructures: local versus nonlocal response," Proc. SPIE, vol. 7757, pp. 188-194, 2010.

[317] M. Kupresak, X. Zheng, G. A. E. Vandenbosch, and V. V. Moshchalkov, "Comparison of hydrodynamic models for the electromagnetic nonlocal response of nanoparticles," Adv. Theory Simul., vol. 1, no. 12, p. 1800076, 2018.

[318] M. Kupresak, X. Zheng, G. A. E. Vandenbosch, and V. V. Moshchalkov, "Appropriate nonlocal hydrodynamic models for the characterization of deep-nanometer scale plasmonic scatterers," Adv. Theory Simul., vol. 2, p. 1900172, 2019.

[319] J. B. Pendry, A. Aubry, D. R. Smith, and S. A. Maier, "Transformation optics and subwavelength control of light," Science, vol. 337, pp. 549-552, 2012. 
[320] Q. Huang, F. Bao, and S. He, "Nonlocal effects in a hybrid plasmonic waveguide for nanoscale confinement," Opt. Express, vol. 21, no. 2, pp. 1430-1439, 2013.

[321] W. A. Davis and C. M. Krowne, "The effects of drift and diffusion in semiconductors on plane wave interaction at interfaces," IEEE Trans. Antennas Propag., vol. 36, no. 1, pp. 97-103, 1988.

[322] G. W. Hanson, "Drift-diffusion: a model for teaching spatial-dispersion concepts and the importance of screening in nanoscale structures," IEEE Antenn. Propag. Mag., vol. 52, no. 5, pp. 198-207, 2010.

[323] N. Schmitt, C. Scheid, S. Lanteri, A. Moreau, and J. Viquerat, "A DGTD method for the numerical modeling of the interaction of light with nanometer scale metallic structures taking into account non-local dispersion effects," J. Comput. Phys., vol. 316, pp. 396-415, 2016.

[324] C. Ciracì, "Current-dependent potential for nonlocal absorption in quantum hydrodynamic theory," Phys. Rev. B, vol. 95, p. 245434, 2017.

[325] H. M. Baghramyan, F. Della Sala, and C. Ciracì, "Laplacian-level quantum hydrodynamic theory for plasmonics," Phys. Rev. X, vol. 11, p. 011049, 2021

[326] W. Ekardt, "Dynamical polarizability of small metal particles: self-consistent spherical jellium background model," Phys. Rev. Lett., vol. 52, pp. 1925-1928, 1984.

[327] D. E. Beck, "Self-consistent calculation of the collective excitations in neutral and charged jellium microspheres," Phys. Rev. B, vol. 43, pp. 7301-7304, 1991.

[328] P. J. Feibelman, "Perturbation of surface plasmon dispersion by "extra" electrons near a surface," Surf. Sci., vol. 282, no. 1, pp. 129-136, 1993.

[329] D. Langreth and H. Suhl, Many-Body Phenomena at Surfaces, Orlando, Academic Press, 1984.

[330] J. Kong, A. J. Shvonski, and K. Kempa, "Nonlocal response with local optics,” Phys. Rev. B, vol. 97, p. 165423, 2018.

[331] F. J. García de Abajo, R. Sapienza, M. Noginov, et al., "Plasmonic and new plasmonic materials: general discussion," Faraday Discuss., vol. 178, pp. 123-149, 2015.

[332] C. Tserkezis, A. T. M. Yeşilyurt, J.-S. Huang, and N. A. Mortensen, "Circular dichroism in nanoparticle helices as a template for assessing quantum-informed models in plasmonics," ACS Photonics, vol. 5, no. 12, pp. 5017-5024, 2018.

[333] N. F. van Hulst, "Plasmon quantum limit exposed," Nat. Nanotechnol., vol. 7, pp. 775-777, 2012.

[334] R. Berndt, J. K. Gimzewski, and P. Johansson, "Inelastic tunneling excitation of tip-induced plasmon modes on noble-metal surfaces," Phys. Rev. Lett., vol. 67, pp. 3796-3799, 1991.

[335] D. R. Ward, F. Hüser, F. Pauly, J. C. Cuevas, and D. Natelson, "Optical rectification and field enhancement in a plasmonic nanogap," Nat. Nanotechnol., vol. 5, pp. 732-736, 2010.

[336] E. H. Hauge and J. A. Støvneng, "Tunneling times: a critical review," Rev. Mod. Phys., vol. 61, pp. 917-936, 1989.

[337] T. Golestanizadeh, A. Zarifi, T. Jalali, J. R. Maack, and M. Wubs, "Hydrodynamic acoustic plasmon resonances in semiconductor nanowires and their dimers,"J. Opt. Soc. Am. B, vol. 36, no. 10, pp. 2712-2720, 2019.
[338] V. M. Agranovich and V. L. Ginzburg, "Crystal optics with spatial dispersion and excitons," in Springer Series in Solid-State Sciences, vol. 42, Berlin, Springer International Publishing, 1984, pp. 1-327.

[339] C. G. Smith, "Low-dimensional quantum devices," Rep. Prog. Phys., vol. 59, no. 2, pp. 235-282, 1996.

[340] F. Stern, "Polarizability of a two-dimensional electron gas," Phys. Rev. Lett., vol. 18, pp. 546-548, 1967.

[341] L. Kong, B. Yan, and X. Hu, "Dispersion relations of longitudinal plasmons in one, two and three dimensional electron gas of metals," Plasma Sci. Technol., vol. 9, pp. 519-525, 2007.

[342] A. Boltasseva and V. M. Shalaev, "Transdimensional photonics," ACS Photonics, vol. 6, no. 1, pp. 1-3, 2019.

[343] S. Boroviks, C. Wolff, J. Linnet, et al., "Interference in edge-scattering from monocrystalline gold flakes [invited]," Opt. Mater. Express, vol. 8, no. 12, pp. 3688-3697, 2018.

[344] Z. M. Abd El-Fattah, V. Mkhitaryan, J. Brede, et al., "Plasmonics in atomically thin crystalline silver films," ACS Nano, vol. 13, no. 7, pp. 7771-7779, 2019.

[345] R. A. Maniyara, D. Rodrigo, R. Yu, et al., "Tunable plasmons in ultrathin metal films," Nat. Photonics, vol. 13, no. 5, pp. 328-333, 2019.

[346] G. Wang, A. Chernikov, M. M. Glazov, et al., "Colloquium: excitons in atomically thin transition metal dichalcogenides," Rev. Mod. Phys., vol. 90, p. 021001, 2018.

[347] A. H. Castro Neto, F. Guinea, N. M. R. Peres, K. S. Novoselov, and A. K. Geim, "The electronic properties of graphene," Rev. Mod. Phys., vol. 81, pp. 109-162, 2009.

[348] S. Thongrattanasiri, A. Manjavacas, and F. J. García de Abajo, "Quantum finite-size effects in graphene plasmons," ACS Nano, vol. 6, pp. 1766-1775, 2012.

[349] W. Wang, T. Christensen, A.-P. Jauho, K. S. Thygesen, M. Wubs, and N. A. Mortensen, "Plasmonic eigenmodes in individual and bow-tie graphene nanotriangles," Sci. Rep., vol. 5, p. 9535, 2015.

[350] M. Settnes, J. R. M. Saavedra, K. S. Thygesen, A.-P. Jauho, F. J. García de Abajo, and N. A. Mortensen, "Strong plasmon-phonon splitting and hybridization in 2D materials revealed through a self-energy approach," ACS Photonics, vol. 4, no. 11, pp. 2908-2915, 2017.

[351] K. O. Wedel, N. A. Mortensen, K. S. Thygesen, and M. Wubs, "Emergent scale invariance of nonclassical plasmons in graphene nanoribbons," Phys. Rev. B, vol. 98, p. 155412, 2018.

[352] E. Galiffi, P. A. Huidobro, P. A. D. Gonçalves, N. A. Mortensen, and J. B. Pendry, "Probing graphene's nonlocality with singular metasurfaces," Nanophotonics, vol. 9, p. 309 , 2020.

[353] A. J. Chaves, N. M. R. Peres, G. Smirnov, and N. A. Mortensen, "Hydrodynamic model approach to the formation of plasmonic wakes in graphene," Phys. Rev. B, vol. 96, p. 195438, 2017.

[354] D. Svintsov, "Fate of an electron beam in graphene: Coulomb relaxation or plasma instability," Phys. Rev. $B$, vol. 101, p. 235440, 2020.

[355] C. Wolff and N. A. Mortensen, "Stimulated plasmon polariton scattering," Nat. Commun., vol. 11, p. 4039, 2020. 
[356] T. Christensen, A.-P. Jauho, M. Wubs, and N. A. Mortensen, "Localized plasmons in graphene-coated nanospheres," Phys. Rev. B, vol. 91, p. 125414, 2015.

[357] P. A. D. Gonçalves, L. P. Bertelsen, S. Xiao, and N. A. Mortensen, "Plasmon-exciton polaritons in two-dimensional semiconductor/metal interfaces," Phys. Rev. B, vol. 97, p. 041402(R), 2018.

[358] E. J. C. Dias, D. A. Iranzo, P. A. D. Gonçalves, et al., "Probing nonlocal effects in metals with graphene plasmons," Phys. Rev. B, vol. 97, p. 245405, 2018.

[359] A. T. Costa, P. A. D. Gonçalves, D. N. Basov, F. H. L. Koppens, N. A. Mortensen, and N. M. R. Peres, "Harnessing ultraconfined graphene plasmons to probe the electrodynamics of superconductors," Proc. Natl. Acad. Sci. U.S.A., vol. 118, no. 4, p. e2012847118, 2021.

[360] M. E. Berkowitz, B. S. Y. Kim, G. Ni, et al., "Hyperbolic cooper-pair polaritons in planar graphene/cuprate plasmonic cavities," Nano Lett., vol. 21, no. 1, pp. 308-316, 2021.

[361] D. N. Basov, A. Asenjo-Garcia, P. J. Schuck, X. Zhu, and A. Rubio, "Polariton panorama," Nanophotonics, vol. 10, no. 1, pp. 549-577, 2021.

[362] K. M. McPeak, S. V. Jayanti, S. J. P. Kress, et al., "Plasmonic films can easily be better: rules and recipes," ACS Photonics, vol. 2, no. 3, pp. 326-333, 2015.

[363] J.-S. Huang, V. Callegari, P. Geisler, et al., "Atomically flat single-crystalline gold nanostructures for plasmonic nanocircuitry," Nat. Commun., vol. 1, p. 150, 2010.

[364] B. Hoffmann, M. Y. Bashouti, T. Feichtner, et al., "New insights into colloidal gold flakes: structural investigation, micro-ellipsometry and thinning procedure towards ultrathin monocrystalline layers," Nanoscale, vol. 8, pp. 4529-4536, 2016.

[365] R. Méjard, A. Verdy, O. Demichel, et al., "Advanced engineering of single-crystal gold nanoantennas," Opt. Mater. Express, vol. 7, no. 4, pp. 1157-1168, 2017.

[366] G. Spektor, D. Kilbane, A. K. Mahro, et al., "Revealing the subfemtosecond dynamics of orbital angular momentum in nanoplasmonic vortices," Science, vol. 355, no. 6330, pp. 1187-1191, 2017.

[367] B. Frank, P. Kahl, D. Podbiel, et al., "Short-range surface plasmonics: localized electron emission dynamics from a 60-nm spot on an atomically flat single-crystalline gold surface," Sci. Adv., vol. 3, p. e1700721, 2017.

[368] S. Boroviks, F. Todisco, N. A. Mortensen, and S. I. Bozhevolnyi, "Use of monocrystalline gold flakes for gap plasmon-based metasurfaces operating in the visible," Opt. Mater. Express, vol. 9, no. 11, pp. 4209-4217, 2019.

[369] J. E. Inglesfield, "Surface electronic structure," Rep. Prog. Phys., vol. 45, no. 3, pp. 223-284, 1982.

[370] W. Shockley, "On the surface states associated with a periodic potential," Phys. Rev., vol. 56, pp. 317-323, 1939.

[371] I. Tamm, "On the possible bound states of electrons on a crystal surface," Phys. Z. Soviet Union, vol. 1, p. 733, 1932.

[372] Z. Quan, Y. Wang, and J. Fang, "High-index faceted noble metal nanocrystals," Acc. Chem. Res., vol. 46, no. 2, pp. 191-202, 2013.

[373] V. Myroshnychenko, E. Carbó-Argibay, I. Pastoriza-Santos, J. Pérez-Juste, L. M. Liz-Marzán, and F. J. García de Abajo,
"Modeling the optical response of highly faceted metal nanoparticles with a fully 3D boundary element method," Adv. Mater., vol. 20, no. 22, pp. 4288-4293, 2008.

[374] J. H. Yoon, F. Selbach, L. Schumacher, J. Jose, and S. Schlücker, "Surface plasmon coupling in dimers of gold nanoparticles: experiment and theory for ideal (spherical) and nonideal (faceted) building blocks," ACS Photonics, vol. 6, no. 3, pp. 642-648, 2019.

[375] P. M. Echenique, J. Osma, M. Machado, V. M. Silkin, E. V. Chulkov, and J. M. Pitarke, "Surface-state electron dynamics in noble metals," Prog. Surf. Sci., vol. 67, nos. 1-8, pp. 271-283, 2001.

[376] B. Diaconescu, K. Pohl, L. Vattuone, et al., "Low-energy acoustic plasmons at metal surfaces," Nature, vol. 448, no. 7149, pp. 57-59, 2007.

[377] A. Politano, V. M. Silkin, I. A. Nechaev, et al., "Interplay of surface and Dirac plasmons in topological insulators: the case of $\mathrm{Bi}_{2} \mathrm{Se}_{3}$," Phys. Rev. Lett., vol. 115, p. 216802, 2015.

[378] M. Autore and R. Hillenbrand, "What momentum mismatch," Nat. Nanotechnol., vol. 14, p. 308, 2019.

[379] D. A. Iranzo, S. Nanot, E. J. C. Dias, et al., "Probing the ultimate plasmon confinement limits with a van der Waals heterostructure," Science, vol. 360, pp. 291-295, 2018.

[380] P. J. Feibelman, "Comment on 'Surface plasmon dispersion of Ag'," Phys. Rev. Lett., vol. 72, pp. 788, 1994.

[381] A. Politano, V. Formoso, and G. Chiarello, "Dispersion and damping of gold surface plasmon," Plasmonics, vol. 3, no. 4, p. 165, 2008.

[382] A. Manjavacas and F. J. García de Abajo, "Tunable plasmons in atomically thin gold nanodisks," Nat. Commun., vol. 5, p. 3548, 2014.

[383] A. J. Wilson and K. A. Willets, "Molecular plasmonics," Annu. Rev. Anal. Chem., vol. 9, no. 1, pp. 27-43, 2016.

[384] A. Lauchner, A. E. Schlather, A. Manjavacas, et al., "Molecular plasmonics," Nano Lett., vol. 15, no. 9, pp. 6208-6214, 2015.

[385] L. Bursi, A. Calzolari, S. Corni, and E. Molinari, "Quantifying the plasmonic character of optical excitations in nanostructures," ACS Photonics, vol. 3, no. 4, pp. 520-525, 2016.

[386] R. Zhang, L. Bursi, J. D. Cox, et al., "How to identify plasmons from the optical response of nanostructures," ACS Nano, vol. 11, no. 7, pp. 7321-7335, 2017.

[387] M. M. Müller, M. Kosik, M. Pelc, et al., “Energy-based plasmonicity index to characterize optical resonances in nanostructures," J. Phys. Chem. C, vol. 124, no. 44, pp. 24331-24343, 2020.

[388] M. M. Müller, M. Kosik, M. Pelc, et al., "From single-particle-like to interaction-mediated plasmonic resonances in graphene nanoantennas,"J. Appl. Phys., vol. 129, no. 9, p. 093103, 2021.

[389] K. A. Willets and R. P. Van Duyne, "Localized surface plasmon resonance spectroscopy and sensing," Annu. Rev. Phys. Chem., vol. 58, no. 1, pp. 267-297, 2007.

[390] K. M. Mayer and J. H. Hafner, "Localized surface plasmon resonance sensors," Chem. Rev., vol. 111, no. 6, pp. 3828-3857, 2011.

[391] A. G. Brolo, "Plasmonics for future biosensors," Nat. Photonics, vol. 6, no. 11, pp. 709-713, 2012. 
[392] H. Hövel, S. Fritz, A. Hilger, U. Kreibig, and M. Vollmer, "Width of cluster plasmon resonances: bulk dielectric functions and chemical interface damping," Phys. Rev. B, vol. 48, pp. 18178-18188, 1993.

[393] N. D. Lang and W. Kohn, "Theory of metal surfaces: induced surface charge and image potential," Phys. Rev. B, vol. 7, pp. 3541-3550, 1973.

[394] B. N. J. Persson and P. Apell, "Sum rules for surface response functions with application to the van der Waals interaction between an atom and a metal," Phys. Rev. B, vol. 27, pp. 6058-6065, 1983.

[395] B. N. J. Persson and E. Zaremba, "Electron-hole pair production at metal surfaces," Phys. Rev. B, vol. 31, pp. 1863-1872, 1985.

[396] S. Peng, J. M. McMahon, G. C. Schatz, S. K. Gray, and Y. Sun, "Reversing the size-dependence of surface plasmon resonances," Proc. Natl. Acad. Sci. U.S.A., vol. 107, no. 33, pp. 14530-14534, 2010.

[397] B. Foerster, V. A. Spata, E. A. Carter, C. Sönnichsen, and S. Link, "Plasmon damping depends on the chemical nature of the nanoparticle interface," Sci. Adv., vol. 5, no. 3, p. eaav0704, 2019.

[398] L. Jauffred, A. Samadi, H. Klingberg, P. M. Bendix, and L. B. Oddershede, "Plasmonic heating of nanostructures," Chem. Rev., vol. 119, no. 13, pp. 8087-8130, 2019.

[399] S. Lal, S. E. Clare, and N. J. Halas, "Nanoshell-enabled photothermal cancer therapy: impending clinical impact," Acc. Chem. Res., vol. 41, no. 12, pp. 1842-1851, 2008.

[400] X. Zhu, C. Vannahme, E. Højlund-Nielsen, N. A. Mortensen, and A. Kristensen, "Plasmonic colour laser printing," Nat. Nanotechnol., vol. 11, no. 4, pp. 325-329, 2016.

[401] M. L. Brongersma, N. J. Halas, and P. Nordlander, "Plasmon-induced hot carrier science and technology," Nat. Nanotechnol., vol. 10, no. 1, pp. 25-34, 2015.

[402] D. M. Newns, "Self-consistent model of hydrogen chemisorption," Phys. Rev., vol. 178, pp. 1123-1135, 1969.

[403] D. Xu, X. Xiong, L. Wu, et al., "Quantum plasmonics: new opportunity in fundamental and applied photonics," Adv. Opt Photon, vol. 10, no. 4, pp. 703-756, 2018.

[404] D. E. Chang, A. S. Sørensen, P. R. Hemmer, and M. D. Lukin, "Quantum optics with surface plasmons," Phys. Rev. Lett., vol. 97, p. 053002, 2006.

[405] D. E. Chang, A. S. Sørensen, E. A. Demler, and M. D. Lukin, "A single-photon transistor using nanoscale surface plasmons," Nat. Phys., vol. 3, no. 11, pp. 807-812, 2007.

[406] D. E. Chang, A. S. Sørensen, P. R. Hemmer, and M. D. Lukin, "Strong coupling of single emitters to surface plasmons," Phys. Rev. B, vol. 76, p. 035420, 2007.

[407] A. Huck, S. Smolka, P. Lodahl, et al., "Demonstration of quadrature-squeezed surface plasmons in a gold waveguide," Phys. Rev. Lett., vol. 102, p. 246802, 2009.

[408] A. González-Tudela, D. Martin-Cano, E. Moreno, L. Martín-Moreno, C. Tejedor, and F. J. García-Vidal, "Entanglement of two qubits mediated by one-dimensional plasmonic waveguides," Phys. Rev. Lett., vol. 106, p. 020501, 2011.

[409] A. González-Tudela, P. A. Huidobro, L. Martín-Moreno, C. Tejedor, and F. J. García-Vidal, “Theory of strong coupling between quantum emitters and propagating surface plasmons," Phys. Rev. Lett., vol. 110, p. 126801, 2013.

[410] R. W. Heeres, L. P. Kouwenhoven, and V. Zwiller, "Quantum interference in plasmonic circuits," Nat. Nanotechnol., vol. 8, no. 10, pp. 719-722, 2013.

[411] J. S. Fakonas, H. Lee, Y. A. Kelaita, and H. A. Atwater, "Two-plasmon quantum interference," Nat. Photonics, vol. 8, no. 4, pp. 317-320, 2014

[412] R. Sáez-Blázquez, J. Feist, A. I. Fernández-Domínguez, and F. J. García-Vidal, "Enhancing photon correlations through plasmonic strong coupling," Optica, vol. 4, no. 11, pp. 1363-1367, 2017.

[413] F. Marquier, C. Sauvan, and J.-J. Greffet, "Revisiting quantum optics with surface plasmons and plasmonic resonators," ACS Photonics, vol. 4, no. 9, pp. 2091-2101, 2017.

[414] I. D’Amico, D. G. Angelakis, F. Bussières, et al., "Nanoscale quantum optics," Riv. Nuovo Cimento, vol. 42, pp. 153-195, 2019.

[415] S. N. Gupta, O. Bitton, T. Neuman, et al., "Complex plasmon-exciton dynamics revealed through quantum dot light emission in a nanocavity," Nat. Commun., vol. 12, p. 1310, 2021.

[416] M. Pelton, "Modified spontaneous emission in nanophotonic structures," Nat. Photonics, vol. 9, p. 427, 2015.

[417] P. Lodahl, S. Mahmoodian, and S. Stobbe, "Interfacing single photons and single quantum dots with photonic nanostructures," Rev. Mod. Phys., vol. 87, pp. 347-400, 2015.

[418] S. I. Bozhevolnyi and J. B. Khurgin, "Fundamental limitations in spontaneous emission rate of single-photon sources," Optica, vol. 3, no. 12, pp. 1418-1421, 2016.

[419] A. V. Akimov, A. Mukherjee, C. L. Yu, et al., "Generation of single optical plasmons in metallic nanowires coupled to quantum dots," Nature, vol. 450, no. 7168, pp. 402-406, 2007.

[420] S. Kumar, A. Huck, and U. L. Andersen, "Efficient coupling of a single diamond color center to propagating plasmonic gap modes," Nano Lett., vol. 13, no. 3, pp. 1221-1225, 2013.

[421] E. Bermúdez-Ureña, C. Gonzalez-Ballestero, M. Geiselmann, et al., "Coupling of individual quantum emitters to channel plasmons," Nat. Commun., vol. 6, p. 7883, 2015.

[422] H. Siampour, S. Kumar, V. A. Davydov, L. F. Kulikova, V. N. Agafonov, and S. I. Bozhevolnyi, "On-chip excitation of single germanium vacancies in nanodiamonds embedded in plasmonic waveguides," Light Sci. Appl., vol. 7, p. 61, 2018.

[423] G. G. Akselrod, C. Argyropoulos, T. B. Hoang, et al., "Probing the mechanisms of large Purcell enhancement in plasmonic nanoantennas," Nat. Photonics, vol. 8, p. 835, 2014.

[424] T. B. Hoang, G. M. Akselrod, C. Argyropoulos, J. Huang, D. R. Smith, and M. H. Mikkelsen, "Ultrafast spontaneous emission source using plasmonic nanoantennas," Nat. Commun., vol. 6, p. 7788, 2015.

[425] T. B. Hoang, G. M. Akselrod, and M. H. Mikkelsen, "Ultrafast room-temperature single photon emission from quantum dots coupled to plasmonic nanocavities," Nano Lett., vol. 16, no. 1 , pp. $270-275,2016$

[426] R. Chikkaraddy, B. de Nijs, F. Benz, et al., "Single-molecule strong coupling at room temperature in plasmonic 
nanocavities," Nature, vol. 535, no. 7610, pp. 127-130, 2016.

[427] S. K. H. Andersen, S. Kumar, and S. I. Bozhevolnyi, "Ultrabright linearly polarized photon generation from a nitrogen vacancy center in a nanocube dimer antenna," Nano Lett., vol. 17, no. 6, pp. 3889-3895, 2017.

[428] Y. Luo, G. D. Shepard, J. Ardelean, et al., "Deterministic coupling of site-controlled quantum emitters in monolayer WSe ${ }_{2}$ to plasmonic nanocavities," Nat. Nanotechnol., vol. 13, no. 12, pp. 1137-1142, 2018.

[429] P. Anger, P. Bharadwaj, and L. Novotny, "Enhancement and quenching of single-molecule fluorescence," Phys. Rev. Lett., vol. 96, p. 113002, 2006.

[430] A. Delga, J. Feist, J. Bravo-Abad, and F. J. García-Vidal, "Quantum emitters near a metal nanoparticle: strong coupling and quenching," Phys. Rev. Lett., vol. 112, p. 253601, 2014.

[431] M. L. Andersen, S. Stobbe, A. S. Sørensen, and P. Lodahl, "Strongly modified plasmon-matter interaction with mesoscopic quantum emitters," Nat. Phys., vol. 7, no. 3, pp. 215-218, 2011.

[432] R. Filter, C. Bösel, G. Toscano, F. Lederer, and C. Rockstuhl, "Nonlocal effects: relevance for the spontaneous emission rates of quantum emitters coupled to plasmonic structures," Opt. Lett., vol. 39, no. 21, pp. 6118-6121, 2014.

[433] C. Tserkezis, N. Stefanou, M. Wubs, and N. A. Mortensen, "Molecular fluorescence enhancement in plasmonic environments: exploring the role of nonlocal effects," Nanoscale, vol. 8, pp. 17532-17541, 2016.

[434] C. Tserkezis, N. A. Mortensen, and M. Wubs, "How nonlocal damping reduces plasmon-enhanced fluorescence in ultranarrow gaps," Phys. Rev. B, vol. 96, p. 085413, 2017.

[435] C. Tserkezis, M. Wubs, and N. A. Mortensen, "Robustness of the Rabi splitting under nonlocal corrections in plexcitonics," ACS Photonics, vol. 5, no. 1, pp. 133-142, 2018.

[436] C. Ciracì, R. Jurga, M. Khalid, and F. Della Sala, "Plasmonic quantum effects on single-emitter strong coupling," Nanophotonics, vol. 8, no. 10, pp. 1821-1833, 2019.

[437] G. P. Zouros, G. D. Kolezas, N. A. Mortensen, and C. Tserkezis, "Monitoring strong coupling in nonlocal plasmonics with electron spectroscopies," Phys. Rev. B, vol. 101, p. 085416, 2020

[438] V. Karanikolas, I. Thanopulos, J. D. Cox, et al., "Weak and strong-coupling dynamics of quantum emitters in the vicinity of metallic films: role of electron spill-out," 2021. arXiv:2102.10832 [Online]. Available at: https://arxiv.org/ abs/2102.10832.

[439] P. T. Kristensen and S. Hughes, "Modes and mode volumes of leaky optical cavities and plasmonic nanoresonators," ACS Photonics, vol. 1, no. 1, pp. 2-10, 2014.

[440] P. Lalanne, W. Yan, K. Vynck, C. Sauvan, and J.-P. Hugonin, "Light interaction with photonic and plasmonic resonances," Laser Photon. Rev., vol. 12, no. 5, p. 1700113, 2018.

[441] S. Franke, S. Hughes, M. K. Dezfouli, et al., "Quantization of quasinormal modes for open cavities and plasmonic cavity quantum electrodynamics," Phys. Rev. Lett., vol. 122, p. 213901, 2019.
[442] M. K. Dezfouli, C. Tserkezis, N. A. Mortensen, and S. Hughes, "Nonlocal quasinormal modes for arbitrarily shaped three-dimensional plasmonic resonators," Optica, vol. 4, no. 12, pp. 1503-1509, 2017.

[443] K. Ding and C. T. Chan, "An eigenvalue approach to quantum plasmonics based on a self-consistent hydrodynamics method,"J. Phys.: Condens. Matter, vol. 30, no. 8, p. 084007 , 2018.

[444] A. M. Urbas, Z. Jacob, L. D. Negro, et al., "Roadmap on optical metamaterials,"J. Opt., vol. 18, no. 9, p. 093005, 2016.

[445] P. A. Belov, R. Marqués, S. I. Maslovski, et al., "Strong spatial dispersion in wire media in the very large wavelength limit," Phys. Rev. B, vol. 67, p. 113103, 2003.

[446] M. G. Silveirinha, "Nonlocal homogenization model for a periodic array of $\epsilon$-negative rods," Phys. Rev. E, vol. 73, p. 046612, 2006

[447] S. I. Maslovski and M. G. Silveirinha, "Nonlocal permittivity from a quasistatic model for a class of wire media," Phys. Rev. B, vol. 80, p. 245101, 2009.

[448] G. Castaldi, V. Galdi, A. Alù, and N. Engheta, "Nonlocal transformation optics," Phys. Rev. Lett., vol. 108, p. 063902, 2012.

[449] S. Savoia, G. Castaldi, and V. Galdi, "Optical nonlocality in multilayered hyperbolic metamaterials based on Thue-Morse superlattices," Phys. Rev. B, vol. 87, p. 235116, 2013.

[450] G. W. Hanson, M. G. Silveirinha, P. Burghignoli, and A. B. Yakovlev, "Non-local susceptibility of the wire medium in the spatial domain considering material boundaries," New J. Phys., vol. 15, no. 8, p. 083018, 2013.

[451] M. Moccia, G. Castaldi, V. Galdi, A. Alù, and N. Engheta, "Dispersion engineering via nonlocal transformation optics," Optica, vol. 3, no. 2, pp. 179-188, 2016.

[452] B. Wells, P. Ginzburg, V. A. Podolskiy, and A. V. Zayats, "Spontaneous emission in nonlocal metamaterials with spatial dispersion," in Springer Series in Solid-State Sciences, vol. 185, Berlin, Springer International Publishing, 2017, pp. 237-277.

[453] A. Khrabustovskyi, K. Mnasri, M. Plum, C. Stohrer, and C. Rockstuhl, "Interface conditions for a metamaterial with strong spatial dispersion," 2017. arXiv:1710.03676 [Online] Available at: https://arxiv.org/abs/1710.03676.

[454] W. Yan, N. A. Mortensen, and M. Wubs, "Hyperbolic metamaterial lens with hydrodynamic nonlocal response," Opt. Express, vol. 21, no. 12, pp. 15026-15036, 2013.

[455] C. David, J. Christensen, and N. A. Mortensen, "Spatial dispersion in two-dimensional plasmonic crystals: large blueshifts promoted by diffraction anomalies," Phys. Rev. B, vol. 94, p. 165410, 2016.

[456] C. David, N. A. Mortensen, and J. Christensen, "Perfect imaging, epsilon-near zero phenomena and waveguiding in the scope of nonlocal effects," Sci. Rep., vol. 3, p. 2526, 2013.

[457] J. Feis, K. Mnasri, A. Khrabustovskyi, C. Stohrer, M. Plum, and C. Rockstuhl, "Surface plasmon polaritons sustained at the interface of a nonlocal metamaterial," Phys. Rev. B, vol. 98, p. 115409, 2018. 
[458] J. B. Pendry, L. Martín-Moreno, and F. J. García-Vidal, "Mimicking surface plasmons with structured surfaces," Science, vol. 305, no. 5685, pp. 847-848, 2004.

[459] C. Della Giovampaola and N. Engheta, "Plasmonics without negative dielectrics,” Phys. Rev. B, vol. 93, p. 195152, 2016.

[460] Y. Li, I. Liberal, and N. Engheta, "Structural dispersion-based reduction of loss in epsilon-near-zero and surface plasmon polariton waves," Sci. Adv., vol. 5, no. 10, p. eaav3764, 2019.

[461] L. Lu, J. D. Joannopoulos, and M. Soljačić, "Topological photonics," Nat. Photonics, vol. 8, no. 11, pp. 821-829, 2014.

[462] S. Buddhiraju, Y. Shi, A. Song, et al., "Absence of unidirectionally propagating surface plasmon-polaritons at nonreciprocal metal-dielectric interfaces," Nat. Commun., vol. 11, p. 674, 2020.

[463] S. A. H. Gangaraj and F. Monticone, "Do truly unidirectional surface plasmon-polaritons exist?,” Optica, vol. 6, no. 9, pp. 1158-1165, 2019.

[464] F. Monticone, "A truly one-way lane for surface plasmon polaritons," Nat. Photonics, vol. 14, pp. 461-465, 2020

[465] J. J. Brion, R. F. Wallis, A. Hartstein, and E. Burstein, "Theory of surface magnetoplasmons in semiconductors," Phys. Rev. Lett., vol. 28, pp. 1455-1458, 1972.

[466] Z. Yu, G. Veronis, Z. Wang, and S. Fan, “One-way electromagnetic waveguide formed at the interface between a plasmonic metal under a static magnetic field and a photonic crystal," Phys. Rev. Lett., vol. 100, p. 023902, 2008.
[467] K. L. Tsakmakidis, L. Shen, S. A. Schulz, et al., "Breaking Lorentz reciprocity to overcome the time-bandwidth limit in physics and engineering," Science, vol. 356, no. 6344, pp. 1260-1264, 2017.

[468] D. Jalas, A. Petrov, M. Eich, et al., "What is - and what is not - an optical isolator," Nat. Photonics, vol. 7, no. 8, pp. 579-582, 2013.

[469] D. Floess and H. Giessen, "Nonreciprocal hybrid magnetoplasmonics," Rep. Prog. Phys., vol. 81, no. 11, p. 116401, 2018.

[470] S. Raza, "Probing plasmonic nanostructures with electron energy-loss spectroscopy," ser. Ph.D. thesis, Technical University of Denmark, 2014.

[471] G. Toscano, "Semiclassical theory of nonclocal plasmonic excitation in metallic nanostructures," ser. Ph.D. thesis, Technical University of Denmark, 2013.

[472] T. Christensen, "From classical to quantum plasmonics in three and two dimensions," ser. Springer Theses, Springer International Publishing, 2017.

[473] P. A. D. Gonçalves, "Plasmonics and light-matter interactions in two-dimensional materials and in metal nanostructures," ser. Springer Theses, Springer International Publishing, 2020.

[474] N. A. Mortensen, "Mesoscopic Electrodynamics - the interplay of nanoscale morphology with the quantum and nonlocal response of metals," ser. Dr. Scient. thesis, University of Copenhagen, 2021. 